\title{
EFFECT OF MEDIA ON FEMALE ADOLESCENTS' SATISFACTION WITH THEIR BODY IMAGE
}

\author{
A Dissertation \\ Presented to \\ The Faculty of the Curry School of Education \\ University of Virginia
}

In Partial Fulfillment

of the Requirements for the Degree

Doctor of Philosophy

by

Cynthia A. Cardosi, M.Ed., M.S.S.

August, 2006 
(C) Copyright by

Cynthia A. Cardosi

All rights reserved

August, 2006 


\section{Abstract}

The present study investigated the effect of media on female adolescents' satisfaction with their body image. Female adolescents were recruited at twoyear, four-year, and career colleges in the Piedmont area of Virginia. The sample consisted of 108 college women who were 18 to 21 years of age. The average participant was 19 years old $(M=19.63)$ and overweight according to the Body Mass Index $(M=25.52)$. Three measures assessed female adolescents' perceptions of their body image and the role of media: Figure Rating Scale, Sociocultural Attitudes Towards Appearance Questionnaire-3, and Body Image/Media Survey. This study explored the interactions among current body image, personal ideal body image, and media ideal body image. It also ascertained the influences of sociocultural factors on female adolescents' perceptions of their body image.

Findings indicate that female adolescents perceive media to have little influence in shaping their body image. They believe that the media ideal is considerably thinner than their personal ideal. Female adolescents are heavier than these ideal body images. In fact, their current body image usually reflects their own body shape. Female adolescents' body images are closely related to their personal ideal images even though they are thinner. They want a thinner body than they currently have. Female adolescents whose body image is heavier than their personal ideal generally are dissatisfied with their bodies, 
whereas female adolescents whose body image is similar to their personal ideal generally are satisfied.

Media generally influence female adolescents whose personal ideal is notably thinner than their body image yet similar to the media ideal, and are dissatisfied with their bodies. These adolescents internalize the message from media to be thin, and then feel the pressure from media to be thin.

The thin ideal portrayed by media has little influence on female adolescents' personal ideal. Female adolescents do not perceive the media ideal as representative of their thin ideal. Female adolescents want to be thinner, but not as thin as the media ideal. The thin ideal portrayed by media generally does not influence female adolescents' satisfaction with their body image. 
Department of Educational Leadership, Foundation, and Policy

Curry School of Education

University of Virginia

Charlottesville, Virginia

\section{APPROVAL OF THE DISSERTATION}

This dissertation, Effect of Media on Female Adolescents' Satisfaction with their Body Image, has been approved by the Graduate Faculty of the Curry School of Education in partial fulfillment of the requirements for the degree of Doctor of Philosophy.

$\frac{M \text { Mold Shany }}{\text { Dr. Harold Strang, Advisor }}$
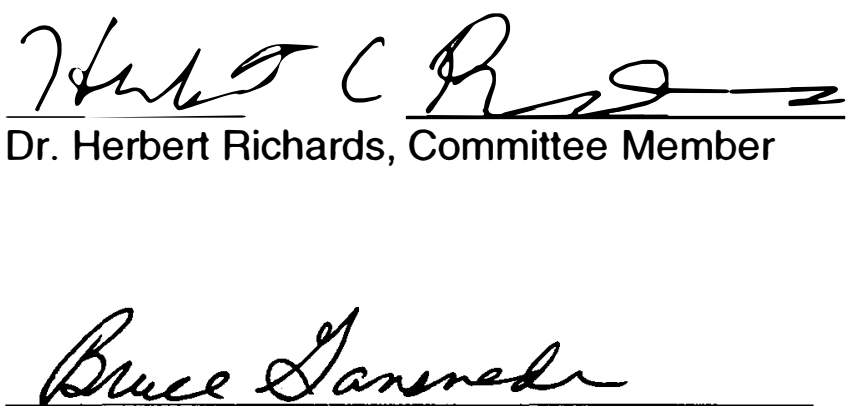

Dr. Bruce Gansneder, Committee Member

Deani Loffinar

Dr. Diane Hoffman, Committee Member

$7-25-06$ Date 
Acknowledgements

Thank you to the young ladies who participated in this study. I appreciated the personal time you took to complete the surveys. Your insights about body image and media were invaluable. My interactions with you have positively touched my life.

This study could not have come to fruition without the support of the administration at the selected sites. Thank you, Tara Atkins-Brady, for granting permission to conduct my research at Piedmont Virginia Community College. I appreciated your time and assistance, especially permitting me to return for additional data. I am grateful to Andreas Anastasiou and Joanna Vondrasek for their support in allowing me to conduct my research at Mary Baldwin College. I also am appreciative that two campuses of the National College of Business and Technology let me conduct my research at their sites. I thank Lew Bishop, Shavvon Gough, Claudia Johnson-Van Hoose, and faculty members for their guidance and time at the Charlottesville campus. At the Lynchburg campus, I thank Sue Coleman for her invaluable service of conducting this study while attending to her own busy schedule. Sue, I truly appreciated your time and assistance.

I am indebted to many people for their guidance and support throughout my graduate experience at the University of Virginia. A special thank you to my 
dissertation committee: Dr. Harold Strang, Dr. Herbert Richards, Dr. Bruce Gansneder, and Dr. Diane Hoffman.

Harry, thank you for being a mentor and advisor during my time at UVa. You inspired me in the classroom as well as out. I am grateful for all the wonderful opportunities accorded me. Thank you for all your unwavering patience, assistance, and guidance from the inception of my research to its conclusion. Your encouragement helped me stay the course.

Herb, thank you for being a mentor throughout my time at Curry. I enjoyed all our lessons in and out of the classroom. I also am appreciative of your advice and suggestions about my dissertation. Thank you for your encouragement especially when my spirits were low.

Bruce, thank you for all the lessons in applied statistics. Your assistance and patience helped me through this research process. You have a great sense of humor.

Diane, thank you for broadening my knowledge of culture. It has been a pleasure working with you. Your expertise was invaluable in enriching this research study.

To my family and friends, thank you for your support and humor during the pursuit of my doctoral degree. Through your encouragement, I made it through this journey. Jeanne, thank you for being there through it all. Our numerous chats at lunch were as wonderful as the food! Kathy thanks for your continual support. You listened when I needed to talk and sounded off when needed. Mom and Dad, thank you for your love and support in everything that I do. 


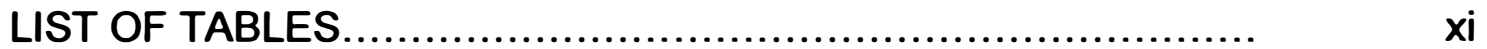

LIST OF FIGURES....................................................

CHAPTER 1. INTRODUCTION....................................

CHAPTER 2. LITERATURE REVIEW.............................

Body Image ............................................... 7

Roots of Body Image Research....................... 8

Body Image Definitions............................... 10

Sociocultural Perspectives.............................. 12

Females......................................... 13

Body Image Perceptions......................... 14

Evolution of the Sociocultural Ideal...................... 15

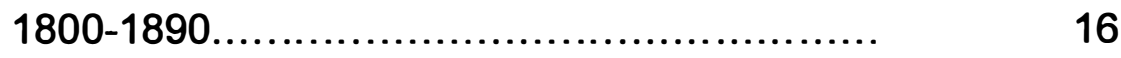

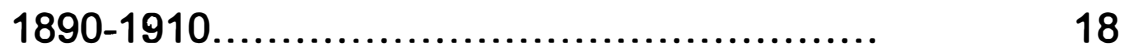

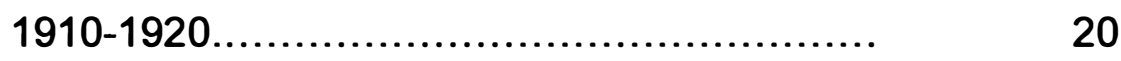

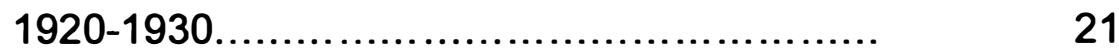

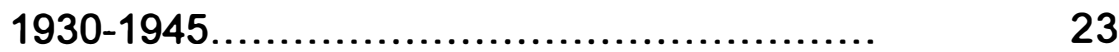

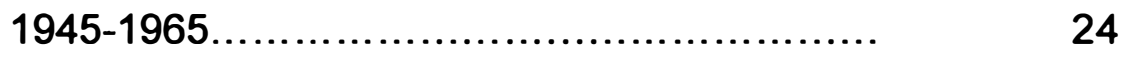

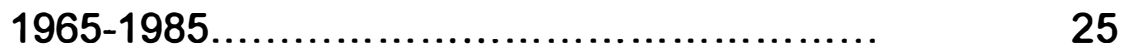

1985 -present................................. 27 
Adolescents.................................................. 30

Development........................................ 30

Body Image .......................................... 31

Experiential Factors........................................... 32

Direct Experiences...................................... $\quad 34$

Family.......................................... 34

Parents................................... 34

Mothers................................... 38

Fathers................................ 40

Siblings.................................. 41

Peers............................................. 42

Friends.................................. 45

Dating Partners.......................... 46

Vicarious Experiences................................ $\quad 49$

Media........................................... 49

Media in general........................... 50

Magazines................................. $\quad 55$

Television................................ 57

Music Videos.............................. 62

Interactions of Direct and Vicarious Experiences......... 66

Summary and Predictions..................................... 
CHAPTER 3. METHOD..............................................

Measures.......................................................

Body Mass Index....................................

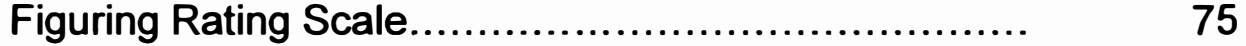

Sociocultural Attitudes Towards Appearance

Questionnaire-3.............................. 77

Body Image/Media Survey.............................. $\quad 78$

Demographics Indices................................... $\quad 79$

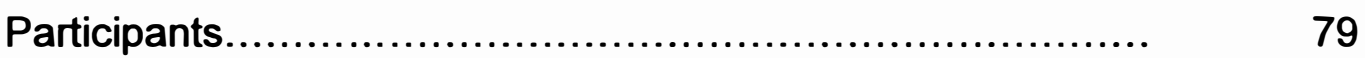

Research Settings............................................ 81

Procedures...................................................... 82

CHAPTER 4. RESULTS ............................................ 84

Demographic Data........................................... 84

Descriptive Data................................................ 86

Figure Rating Scales................................ $\quad 86$

Sociocultural Attitudes Towards Appearance

Questionnaire-3................................. $\quad 87$

Body Image/Media Survey.............................. $\quad 89$

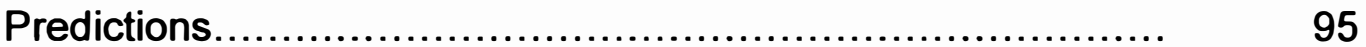

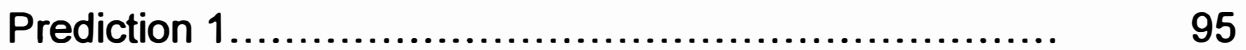

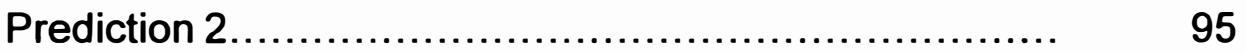

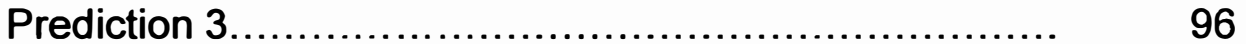




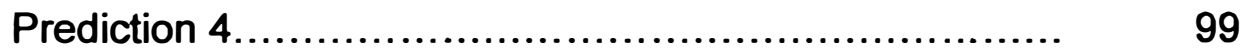

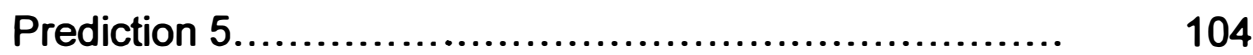

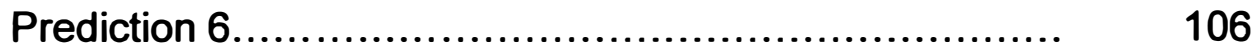

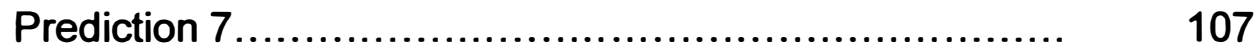

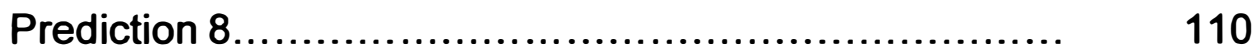

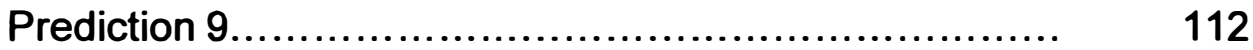

CHAPTER 5. DISCUSSION............................................ 113

Ideal Body Images............................................... $\quad 114$

Body Image and Interactions with Ideal Body Images............. 118

Current Body Image and Personal Ideal................... 118

Current Body Image and Media Ideal...................... 124

Predictors of Body Image Satisfaction..................... 127

Sociocultural Factors......................................... 130

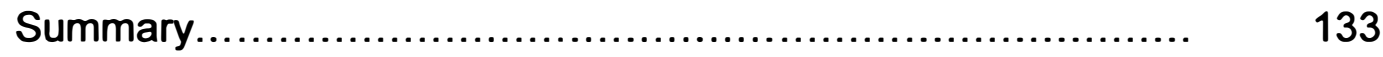

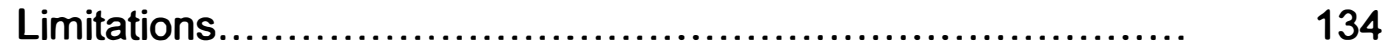

Future Research............................................... 136

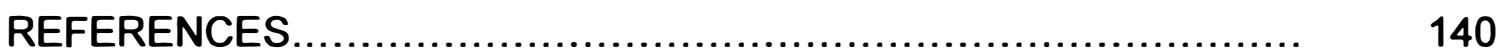

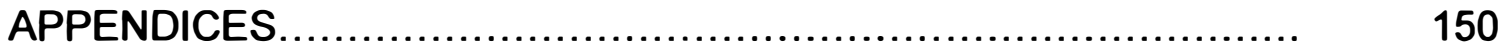

Appendix A - Figure Rating Scales.............................. 150

Appendix B - Sociocultural Attitudes Towards Appearance

Questionnaire-3....................................... 154

Appendix C - Body Image/Media Survey....................... $\quad 157$ 
Appendix D - Demographic Indices.............................. 161

Appendix E - Letter to Administrators............................. 163

Appendix F - Informed Consent Agreement...................... 165

Appendix G - Study Procedures .................................. 167

Appendix H - Site Information Sheet ........................... 170

Appendix I - Interested in the Results Form..................... 172 


\section{LIST OF TABLES}

Tables

Page

1. Means and Standard Deviations of Demographic Indices.......... 85

2. Means and Standard Deviations of Figure Rating Scales.......... 87

3. Means and Standard Deviations of Sociocultural Attitudes

Towards Appearance Questionnaire-3...................... 88

4. Means and Standard Deviations of Body Image/Media Survey.... 90

5. How does the way females appear in media influence how you feel about your body?........................................

6. What is the most powerful message about the female body that media convey to you?

7. Correlations of Personal Ideal Body Image and Current Body Image with Body Image Satisfaction, Media Ideal Influence, SATAQ-3 subscales, Total SATAQ-3, and BMI

8. Percentages and Frequencies of the Items on the Body Image Satisfaction Scale

9. Correlations of Current Body Image and Personal Ideal

Discrepancy with Body Image Satisfaction, Media Ideal Influence, SATAQ-3 subscales, Total SATAQ-3, and BMI 
Tables

10. Intercorrelations of the Current Body Image and Media Ideal

Body Image Discrepancy, Body Image Satisfaction, Media

Ideal Influence, SATAQ-3 subscales, Total SATAQ-3, and BMI

11. Means and Standard Deviations of the Sociocultural Factors in

Rank Order. 


\section{LIST OF FIGURES}

Figures

Page

1. Figure Rating Scale..............................................

2. Body Image Satisfaction.............................................. 101 


\section{CHAPTER 1}

\section{INTRODUCTION}

We currently live at a time and place in history referred to as the Media Age (Singer \& Singer, 2001; Strasburger \& Wilson, 2002; Thompson, Heinberg, Altabe, \& Tantleff-Dunn, 1999). Media essentially permeate the everyday lives of people in American society. They are inundated with media-generated visual images and information. Visual images and information are displayed in magazines and on television, movies, and the Internet. Media have made life more convenient by educating, entertaining, and informing society. Many researchers believe that media have changed the way people view their world (Kitch, 2001; Singer \& Singer, 2001; Strasburger, 1995; Strasburger \& Wilson, 2002; Van Evra, 1998). In particular, visual imagery has made it easier for people to process and retain information, and its impact greater than words (Kitch, 2001; Singer \& Singer, 2001; Strasburger, 1995; Strasburger \& Wilson, 2002; Van Evra, 1998).

Media have become part of the American household $24 \Pi$. The average American in 1998 consumed 11.8 hours of media a day (TV-Turnoff Network Real Vision, 2004). In fact, many Americans have even become obsessed with media, tuning in wherever and whenever they can. When names of celebrities like Katie Moss, Halle Berry, Lindsay Lohan, and Alicia Keyes are encountered, a 
visual image of that celebrity immediately comes to mind. This is precipitated by electronic and print mediums' constant barrage of celebrities' images. Besides the visual images, people repeatedly hear and read details about celebrities' lifestyles. Media have created the celebrity culture and enticed people to learn about celebrities' personal and professional lives by inundating them with visual images and information. For example, there are many television celebrity gossip/news programs and magazines, such as Access Hollywood, Entertainment Weekly, Extra, Insider, People, and TV Guide to name a few, that keep us all abreast of news about celebrities. Some people, though, have been obsessed with the celebrity culture and tune in to learn all that they can about it. They feel like they know the celebrities and see them as "beautiful people" attractive, successful, and living the good life (Grogan, 1999; Kitch, 2001; Strasburger \& Wilson, 2002). Ultimately, some viewers want to emulate them. Many young women want to look like female celebrities (Botta, 1999; Grogan, 1999; Signorelli, 1997; Strasburger \& Wilson, 2002). That is, they want to be attractive, youthful, and thin. This is the prescribed standard of beauty for females in Western society today (Grogan, 1999; Jackson, 1992; Kitch, 2001; Thompson et al., 1999). Undeniably, the majority of female celebrities model this standard. This standard of beauty in which thinness is inordinately emphasized is displayed on a daily basis through media, and they cultivate this thin body image (Silverstein, Perdue, Peterson, \& Kelly, 1986; Thompson et al., 1999).

As a result, celebrities' body images seem real, normal, and attainable to females (Grogan, 1999; Jackson, 1999; Kitch 2001). Unfortunately, these 
images have become progressively thinner over time. Several studies have shown that women are portrayed as abnormally thin in the media. Garner, Garfinkel, Schwartz, and Thompson (1980) were the first investigators to document the new reality; the media ideal body image had shifted from curvaceous to linear. The authors analyzed the weight, height, and measurements of Playboy magazine centerfolds and Miss America pageant contestants from 1959 to 1978 . They found that there was a gradual but significant decline in the weights of both groups over the 20 -year period with a greater decline in the second decade (Garner et al., 1980).

Wiseman and colleagues (Wiseman, Gray, Mosimann, \& Ahrens, 1992) replicated the Garner et al. study by looking at the measurements of the Playboy centerfolds and Miss America contestants from 1979 to 1988 . The authors pointed out that the body weights were found to stabilize at $13-19 \%$ below expected weight with anything below $15 \%$ a concern for an eating disorder according to the Diagnostic and Statistical Manual of Mental Disorders-III-R (Wiseman et al., 1992). Results revealed that there were further decreases in body size of Miss America contestants over this period while the Playboy centerfolds leveled off at a very low level. In another study, Silverstein and colleagues (1986) conducted an analysis of bust-to-waist and hip-to-waist ratios of models from different mediums during 1901 to 1980 . The investigators found that television and movie models were slimmer than in the past. They also found that magazine models have become slimmer and linear for women since the 1930's. In a similar study during 1970-1990, Guillen and Barr (1994) also found 
that the models have become more linear and slimmer. Other studies of the representation of the female body in the media have reliably established that models have become thinner since the 1960s (Grogan, 1999; Jackson 1992). The findings of all these studies converge: The portrayal of the female body in media has become extremely thin, well below the recommended weight for height (Wiseman et al., 1992). Females accept this thin ideal as the standard for beauty even though the ideal body image has become thinner over the past 40 years (Grogan, 1999; Hesse-Biber, 1996; Thompson et al., 1999).

There are several beliefs about being thin that appeal to young women. For instance, they believe that achieving the thin ideal is attractive and will promote happiness and positive self-esteem (Hesse-Biber, 1996; Sault, 1994; Thompson et al., 1999). They will gain control of their lives if they are thin (Kitch, 2001; Sault, 1994). They will be successful (Jackson, 1992; Maine, 2000). Thin females will be accepted in society (Hesse-Biber, 1996; Nichter \& Vuckovic, 1994). They will be liked (Grogan, 1999; Nichter \& Vuckovic, 1994). More important, being thin will attract the opposite sex (Grogan, 1999; Hesse-Biber, 1996; Maine, 2000; Thompson et al., 1999). Media play a major role in promoting such beliefs.

It is through vicarious, subtle, and cumulative communication extolling the thin ideal that media transmit their message to females. Through modeling, media set the standard against which females assess their perceived body image. 
In contrast, the average female has become heavier over the years (Etcoff, 1999; Garner et al., 1980; Hesse-Biber, 1996; Thompson et al., 1999). In fact, $26 \%$ of the female population over 19 years old in America is currently obese and 26\% overweight (Centers for Disease Control and Prevention, 2006). Whereas, the female body portrayed in media is extremely thin (Guillen \& Barr, 1994; Wiseman et al., 1992). Researchers have indicated that it is difficult for females to attain this thin ideal portrayed in media (Garner et al., 1980; Grogan, 1999; Jackson, 1992; Thompson et al., 1999). Yet, females tend to accept this standard and want to achieve it (Grogan, 1999; Hesse-Biber, 1996; Thompson et al., 1999).

It is believed that females perceive their own body image - what one is in relation to a thin ideal like the media portray - what one wants to be. Many body image studies have reported a disparity between the thin ideal portrayed in media and females' personal perceptions of body image (Durkin \& Paxton, 2002; Garner et al., 1980; Grogan, 1999; Nichter \& Vuckovic, 1994; Tiggemann \& Pickering, 1996). This discrepancy has been linked to females' personal dissatisfaction with their bodies (Durkin \& Paxton, 2002; Grogan, 1999; HesseBiber, 1996; Jackson, 1992; Kitch, 2001; Strasburger \& Wilson, 2002; Thompson et al., 1999).

The purpose of this study is to investigate the effect of media on female adolescents' perceptions of their satisfaction with their body image. The present study inquires into how female adolescents' perceive their body image, personal ideal body image, and the media ideal body image. It examines the relationship 
between personal ideal body image and media ideal body image, and probes female adolescents' perceptions of their own body image. Specifically, this study looks at the relationship between female adolescents' perceived body image and their personal ideal body image. More importantly, it delves into the influence of media on female adolescents' perceived body image. 


\section{CHAPTER 2}

\section{LITERATURE REVIEW}

The literature review reveals the complexity of body image, and its importance to females. It tracks the concept of body image from infancy to present day. In this chapter, historical roots of society's view of body image are reviewed, and its importance in the self-concept of females. It, then, traces the history of the cultural ideal body image over the last two centuries. This review continues on to describe late adolescence development along with adolescents concern about their body image, and finally, explores the research of experiential factors that influence female adolescents' body image.

Body Image

Body image is a socioculturally derived entity that is shaped by the self and others' opinion of the self. In order to understand the concept of body image, the journey begins with reviewing past body image research to grasp how the concept of body image has evolved. Various body image terms and definitions from the literature are reviewed, and then for the present study, the sociocultural theory defines and explains body image. From this context, today's standards of beauty for females are described along with making a distinction between the sociocultural ideal body image and perceived body image. The 
body image section concludes with the history of society's ideal body image in America from the 1800 s to present day.

\section{Roots of Body Image Research}

The concept of body image emerged in the social and medical fields at the beginning of the twentieth century. John Horton Cooley (1902) coined the phrase "the reflected or looking glass self" in describing social self-concept. It is interesting to note that the "mirror" metaphor still has been referenced in many present day studies. In Human Nature and the Social Order, Cooley (1902) pointed out that one's social interaction with others help shape the self. He contended that "others" are the social mirror in which one looks to find the opinions towards oneself. Cooley concluded that once others' opinions are accommodated over time, it becomes part of the self.

From the 1900 to 1950 , body image research began in the medical field. The term "body schema" was used because it focused on the body and its connection to brain functions. Neurologists studied brain damage and its effect on the body. They were especially interested in the cause of lost sensations in parts of the body. There were also studies with lost limbs and its effect on the brain, like phantom limb pain. Researchers found that people eventually accommodate to the physical changes (Fisher, 1986). In the 1920s, Schilder (1950), a neurologist, was noted for his work in the psychological self-awareness of body schema. Schilder studied neurological problems and discovered that body attitudes towards one's own body influenced behavior. He broadened the study of body schema, or image as he called it, from neurology to the 
psychoanalytical and sociological disciplines. Moreover, he was the first researcher to show it was possible to apply body image concepts to everyday people. Schilder was the first researcher to acknowledge that body image was a multidimensional concept. He opened the door for the concept of body image to be studied outside of neurology. Beginning in the 1940s and 1950s, body image research broadened to other disciplines. For instance, research in selfperceptions of appearance was introduced. Secord and Jourard constructed one of the first scales to assess self-rating of attractiveness (Thompson et al., 1999). In addition, the psychodynamic perspective of body image research began in the 1950s. Fischer developed the body image boundaries theory that used Rorschach ink blots. He suggested that the concept of body image was served within the personality as a boundary between the self and environment (Shontz, 1993).

During the 1960s, Strunkard and colleagues had begun research in eating disorders and their relationship to body image (Thompson et al., 1999). The participants in their studies were underweight and overweight people. Other researchers joined the ranks and studied disturbed body image. In the late 1960s, Shontz's research focused on the cognitive-behavioral component of body image. His research dealt with the cognitive and perceptual perspectives of body experiences through the physically disabled. Body image research essentially had branched out to many disciplines by the 1960 s.

In recent years, body image research has tripled that of the 1970 s. Over the last century and into the $21^{\text {st }}$ century, body image research has grown 
tenfold. Clinical, medical, psychology, philosophy, anthropology, communication, and sociology disciplines have some aspect of body image research in progress. Body image research has found its place and time in scientific inquiry. Yet, there have been few connections across the disciplines with body image studies (Fisher, 1986; Pruzinsky \& Cash, 2002; Thompson et al., 1999).

\section{Body Image Definitions}

The term body image would seem on the surface easy to define.

However, from the thousands of studies in this field, there have been many ways that it has been presented. One definition, "the mental picture of the body" (Thomas, 1988, p. 1), only provided a physical description of body image. In a survey report, Garner $(1997$, p. 7) defined it as "a mental representation of the self" that denoted a psychological context. Researchers have used various definitions for body image over time, such as a picture of one's self in one's mind, how one feels about one's own body and appearance, mental construction of self, conception of and attitude toward one's physical appearance, physical appearance, and reflections and feelings one has about one's body to name a few. Grogan (1999, p. 1) described body image as "the person's perceptions, thoughts, and feelings about his or her body" - a psychosocial perspective. Thompson et al. (1999, p. 4) defined body image as the "internal representation of your own outer appearance - your own unique perception of your body". Similarly, Cash (1990, p. 51) viewed physical appearance as contrasting the "view from the inside", the physical self-concept, with the "view from the outside", the social object. This internal view has been associated with thoughts and 
feelings, whereas the external view was connected with the social image. These illustrations have been some of the definitions applied in body image research today.

Schilder's (1950) definition, one of the most quoted in body image research, "the picture of our own body which we form in our mind, that is to say, the way in which the body appears to ourselves" $(1950$, p. 11). It was one of the earliest body image definitions adopted by various disciplines. Schilder's definition has been cited in studies to illustrate that his body image concepts were less complex than today's multidimensional ones.

Thompson and colleagues (1999) have indicated that defining body image has been difficult, as there have been too many variations of the term. They also found that researchers sometimes used terms like physical appearance and body image interchangeably. Thompson et al. (1999, p. 10) compiled a list of 15 body image definitions using terms, such as weight satisfaction, size perception accuracy, appearance satisfaction, body esteem, and body image disturbance to list a few. Overall, there has been no consensus in defining body image.

As indicated, body image has been the all-encompassing term for many constructs. Body image has been the umbrella for any term found in body image research. Many researchers have agreed that body image was a multidimensional facet (Grogan, 1999; Jackson, 1992; Pruzinsky \& Cash, 2002; Thompson et al., 1999). Therefore, the focus of any research and its conceptual framework has to be determined in order to appropriately define body image. Pruzinsky and Cash (2002, p. 7) explained, "Ultimately, terminological lucidity 
can be obtained by precisely defining conceptual referents within the context of the multidimensionality of body experience." Accordingly, body image was defined and explained from the sociocultural perspective for the present study.

\section{Sociocultural Perspective}

Sociocultural theory maintains that cultural and social forces impact a person's thinking and behavior (Vygotsky, 1978). According to Jackson (1992, p. 35), "fundamental to the sociocultural perspective is the view that cultural values influence individual values and behaviors." Through social interactions, an individual also is affected by society's beliefs and values. The individual throughout her development adopts these beliefs and values. As human beings, the extent these beliefs and values influence one's perceptions depend on their personal importance. The acquired beliefs and values of culture and society become part of the person's ideology over time. The sociocultural theory essentially provides an understanding of how sociocultural beliefs and values influence a person's perceptions and attitudes (Vygotsky, 1978).

Within the sociocultural framework, body image is a multidimensional construct that consists of perceptual and attitudinal aspects. The term "body image ${ }^{n}$ is defined as a visual mental representation of the body. In other words, it is the visual image of the physical body reflected in the mind. "Body" in body image signifies the shape and size of the physical body. This mental view of body image has been influenced by culture and society throughout a person's lifespan. One carries that image around in one's mind. Granted that, a person's self perception also has an important role in body image construction. Cash and 
Fleming (2002, p. 278) explained, 'What is 'reflected' back to this individual has been created, at least in part, by her own beliefs and their derivative social behaviors." Therefore, the self and sociocultural elements are interfacing when molding the person's body image; but ultimately, it is the individual who determines what aspects of body image are accepted as relevant to her.

\section{Females}

Researchers have claimed that body image has been more important for females than males (Gimlin, 2002; Grogan, 1999; Hesse-Biber, 1996; Jackson, 1992). There have been several reasons why this has been true. One reason has been the task of finding a mate, one who will meet her expectations and provide a good home (Gimlin, 2002; Grogan, 1999; Jackson, 1992). It is believed that heavy women would have a more difficult time finding a mate than thin or average women. Another reason has been that females are relationshiporiented, so body image has made a difference when selecting friends as well as wanting to be liked (Nichter \& Vuckovic, 1994). Females have no desire to be rejected, which tends to be the case when fat (Jackson, 1992; Nichter \& Vuckovic, 1994; Pipher, 1994). In a patriarchal society, females have been valued more for their appearance than their intelligence, and they have heard this message since childhood (Jackson, 1992; Kitch, 2001; Maine, 2000). In Western society, females have been evaluated more often for their appearance than not (Grogan, 1999; Jackson, 1992; Maine, 2000). Attractive females generally have received better treatment in society (Cash \& Fleming, 2002; Grogan, 1999; Jackson, 1992). Most importantly, a positive body image has symbolized taking 
responsibility and control of one's life; something females have desired to achieve for over a century (Grogan, 1999; Sault, 1994; Thompson et al. 1999). Body image perceptions

How do females know whether their body image has met expectations? Sociocultural beliefs and values have been derived by culture and society at that time and place in history. Sociocultural concepts then have influenced the standards of beauty for females in Western society (Grogan, 1999; Jackson, 1992, 2002; Thompson et al., 1999). Jackson (2002) explained that culture has defined what made an appealing body and the self-perception of body appeal has depended on these standards. Presently, the standards of beauty for females in Western society are attractiveness, thinness, and youthfulness (Grogan, 1999; Jackson, 2002, Sault, 1994; Thompson et al., 1999).

Researchers have found these standards associated with happiness, success, and social acceptability (Grogan, 1999; Jackson, 1992). Body image has become the focal point of the standards for beauty; it is now the only malleable characteristic (Grogan, 1999). According to Thompson et al. (1999, p. 85), "Current societal standards for beauty inordinately stress the importance for thinness." Psychologically, thinness equates to being able to manage one's life (Grogan, 1999; Jackson, 1992; Sault, 1994). The accepted sociocultural standard for body image was thinness (Grogan, 1999; Jackson, 1992; Sault, 1994). Thus, a thin body image represents the sociocultural ideal. Henceforth, the sociocultural ideal may also be referred to the thin ideal, physical ideal, media ideal body image or some derivative. The sociocultural ideal body image is 
usually the standard against which females measure their body image. An individual perceives her body image in relation to the sociocultural ideal. This individual's perceived body image also is cultivated by her perceptions of "self" and others' attitudes towards her body. Sault (1994, p. 18) aptly stated, "What is most important for us is how our body image is perceived by us and others, and we continually try to make the perception fit the ideal." The perceived body image also referred to current body image generally has been measured against the sociocultural ideal. How does the perceived body image stack up to the sociocultural ideal body image? The response generally has been measured along a continuum of satisfied to dissatisfied dependent on the perceived body image (Grogan, 1999; Jackson, 1992; Thompson et al., 1999).

Ultimately, the perceived body image and sociocultural ideal body image have created two visual body images in women's mind - what I look like and what I desire to look like. By understanding how these images were perceived, an individual may realize how susceptible she is to sociocultural standards. The question is has this always held true or are females conforming to the sociocultural standards now?

\section{Evolution of the Sociocultural Ideal}

Body image has been a facet of society's standards of beauty. The standards of beauty have been touted throughout American history. Whenever the beauty ideals have changed, females tended to follow suit (Banner, 1983; Kitch, 2001; Steel, 1985; Thompson et al, 1999). They have attempted to emulate the sociocultural ideals for centuries (Jackson, 1992; Kitch, 2001; 
Thompson et al., 1999). By following the history of the sociocultural ideal in America, people have learned how society's standards of beauty have been transformed. Undeniably, the American female beauty ideal has changed over the last two centuries.

In the following periods, each one has presented a general overview of women's role during the time, description of the sociocultural ideal body image, fashion, and the rise of media.

$1800-1890$

The nineteenth century was the Victorian period in America. With the Industrial Revolution, there was a major increase in middle class society. This was a time when women's goals were to be beautiful, please others, and marry. Beauty conveyed moral character as well as physical beauty. The Victorian woman was continually pressed to cultivate her beauty. It was her first duty to society. Beauty was also associated with femininity. As quoted in Steele's Fashion and Eroticism (1985, p. 102), John Stuart Mill wrote, "'The great occupation of women' should be to 'adorn \& beautify' the family home ' $\&$ to diffuse beauty, elegance, \& grace everywhere'." It was not only her duty but also her desire to be beautiful. Victorian young women were raised to attract "good" suitors, marry, and become showpieces for their husbands. During the 1800s, the reproductive figure of the mature women's bodies conveyed beauty (Banner, 1983; Grogan, 1999; Thompson et al., 1999). In essence, a woman with a voluptuous physical figure was considered a beauty even without an attractive face (Steele, 1985). 
The ideal Victorian female figure was full bosomed, small waisted, and large hipped (Banner, 1983; Steel, 1985). Excessive fat was undesirable and unattractive. And thinness was considered worse than obesity. Victorian women were expected to have a curvaceous silhouette, a sign of their beauty. The small waist was extolled and the epitome of the ideal beauty. The corset was a staple for females during the Victorian age, as it compressed their stomachs to produce the "small waist" effect (Kitch, 2001; Steel, 1985). Women were also known to diet to make the fit of the corset more bearable. The aim for women was to try to achieve an eighteen-inch waist, as it was considered stylish (Banner, 1983). The corset was comprised of steel or whalebone and had a lasting affect on some women, such as death or internal problems attributed to tight lacing. Indeed, women endured this pain or worse in order to achieve that feminine physical ideal.

It was through clothing that the female figure was displayed. The fashions for the Victorian woman did not change much over the century. American fashions followed European models. Women were fully clothed so most parts of their bodies were not exposed in society. It was the silhouette that outlined the female figure. The dress during the 1800 to 1820 was empire style with the emphasis on well-endowed breasts. Padding was used when women were not adequately developed. It was thereafter that fashion moved the focus to the waist. From 1825 to 1890 , the corset enhanced the waist for a smaller effect. Moreover, the rest of the curvaceous figure was naturally endowed or padded to 
achieve the effect. Women did what they had to do in order to have an hourglass figure silhouetted.

Originally, the expression of beauty was communicated through art where visual form first revealed women's beauty. As industrialization spread, media had begun its ascent into capturing this expression of beauty and its ideal through print. The new technologies had begun producing fashion plates that imprinted images of how women should appear (Wolf, 2002). Fashion plates were printed in magazines like Godey's Lady Book that began circulation around the 1830s (Kitch, 2001). Illustrations of the "Steel-Engraving Lady" were presented in magazines. The steel-engraving lady, a lithograph image, represented the ideal physical body during the first half of the 1800s (Banner, 1983). Advertisements like soap also had begun using images of the steelengraving lady around mid-century. Magazines grew rapidly after the Civil War with over 700 titles (Kitch, 2001). They made it possible for women of all social classes to follow the dictates of European women, the trendsetters at the time. On the entertainment front around mid to late 1800 s, popularity arose in variety theatre and burlesque. The most popular stage actress at this time was Lillian Russell who weighed over 200 pounds and was the epitome of the American ideal beauty. Between magazines and entertainment, the image of the physical ideal began reaching all classes of women (Banner, 1983; Steel, 1985). $1890-1910$

The last decade of the nineteenth century and early part of the twentieth century defined a period of modernization in America. It was a time of 
"overturning Victorian social repression and achieving new freedoms for women" (Banner, 1983). Women were beginning to enter the workforce in white-collar jobs. Leisure time was spent bicycling, playing tennis, or some other physical activity. Exercise was the new craze and so was dieting; women did all this to get into shape. The new ideal was a healthy and athletic woman. Women's physical maturity was still prized more than the younger immature ones. This was the period of the "New Woman" in America (Banner, 1983; Kitch, 2001; Steel, 1985).

The physical ideal for women during this period was large bosoms, elongated waist, and slimmer hips. The $S$ shaped corset was worn to produce this effect. To emphasis the breasts, the full bosom was thrust forward, the waist pushed in, and the hips back. The physical ideal image was embodied in the "Gibson Girl" created by Charles Gibson for his illustrations in Life magazine during the 1890s (Banner, 1983; Steel, 1985). The Gibson girl was thinner than the 1800 s physical ideal, but still curvaceous. She became the first model of beauty created in America that gained international recognition (Banner, 1983). The Gibson girl became the dominant American beauty ideal.

The fashions during this time showcased the breasts and slimmer hips. Slimmer hips were likely a result of fewer petticoats. Women had begun wearing blouses, skirts and jackets moreso as a result of leisure time. The clothes were more form-fitting. Banner (1983) wrote that skirts became shorter and tight lacing was abandoned over time. The shirtwaist, an American invention, originally was worn by working class women and then spread to other classes. It 
was a mainstay of fashion from this period forward. In fact, manufactured clothing like the shirtwaist was the beginning of ready wear for women in America (Banner, 1983). It grew out of necessity as women worked and did not have time to go to fittings or to make their clothes.

Magazines were not widely distributed until the 1890 s when over 6,000 were in circulation (Kitch, 2001). The Gibson girl was the cover model for most magazines. Moreover, an advertising blitz was launched with the Gibson girl. Even clothes were named after her (Banner, 1983; Steel, 1985). According to Kitch (2001, p. 37), the "Gibson Girl rise to fame created a blueprint for the commercial uses of such a stereotype." Thus, the beginning of the American standard of beauty through media began. $1910-1920$

According to Valerie Steele (1985, p. 224), this period was considered "the dividing line between the $19^{\text {th }}$ century and the modern age of fashion and beauty". Women were striving for more independence and freedom during this Progressive Era. Women suffrage became organized and part of the public domain. It was a time when women of all social classes began interacting. Women continued taking care of their personal and physical selves. Young women were working and expressing themselves more openly, especially through their behavior and style. They were having fun and delaying marriage. The motto for women in America at the time was to "live right, eat right, exercise, and you will be beautiful" (Banner, 1983, p. 203). 
The 1910s fashionable physical ideal was slender, small breasts, slimmer hips, and long legs (Banner, 1983; Steel, 1985). This body type represented youth. Naturalness was fashionable both in face and form. Unfortunately, most women were only beginning the quest to be thin, a "desire more than a reality" (Steele, 1985). Women were trying to lose weight through diet and exercise in order to look good in the form-fitting clothes that had become fashionable. For most females, the girdles replaced the corset and brassieres became commonplace. Women found freedom with wearing fewer clothes. The clothes were more form fitting from skirts and blouses to dresses. Hemline rose and young women were sporting sleeveless, straight shifts.

The media, especially magazine distributions, were expanding during the 1910s. Posters from magazines became popular during World War I, and women graced the front cover three times as often then men (Kitch, 2001). Some magazine artists employed models that fit the physical ideal and used their illustrations for the magazine covers. At the same time, silent movies became popular. Film stars such as Theda Bara and Mary Pickford personified the American physical ideal of the time. The public was exposed to the modern American women as presented on magazine covers and in films. $1920-1930$

This period became known as the Flapper Era (Banner, 1983; Steel, 1985). The $19^{\text {th }}$ Amendment was passed, a major victory for women. Social rebellion and personal independence was the rule of the decade for women (Banner, 1983). It was the dawn of the "New Girl". Celebration of youth was 
excessive during this time. The flapper took time out for dancing and having fun before settling down to marriage. She was always in motion. Women spent more time and energy on their bodies, because the thin ideal was prevalent.

The physical ideal was slimmer yet. She was more vertical with a straight figure and tall (Banner, 1983). The flapper had a boyish body and short hair. She wore a lot of make up. Her long legs were exposed as rolled stockings fell down and her straight shift was just below her knees (Banner, 1983; Steel, 1985). Any women with full breasts who wore this fashion resorted to binding her breasts thereby doing muscular damage. The non-slim woman was not in fashion. Strict dieting was essential to maintain this physique or to try to achieve it. Early findings of eating disorders were diagnosed for some women during this period (Banner, 1983). Despite the health implications, women continued their diet regimes. In the meantime, all women tried to wear some version of the flapper style. There was more freedom of exposing their bodies with the sleeveless, straight shifts especially while doing the "Charleston". Beauty was enhanced when wearing this style on the boyish body (Banner, 1983).

There was a major marketing blitz associated with the flapper. A clothing line was developed. Magazines and novels immortalized the flapper image. In fact, the marketing effort rallied women throughout the country to imitate the flapper image (Banner, 1983; Kitch, 2001; Steel, 1985). Movies showcased the flapper having fun and dancing the night away, always on some man's arm. Most of the 1920s actresses embraced the flapper image. All this marked the 
beginning of advertising and commercializing the standard of beauty in America (Banner, 1983; Kitch, 2001).

$1930-1945$

The Great Depression and World War II caused major changes in lifestyles over 20 years. The nation was in a crisis. The frivolities of the 1920 s passed, and the country became more focused on improving the economy. Men and women acted more conservatively as they survived tough times. It was a time to work together and help one another. During the war years, women stepped in to provide homeland security for America while the men were abroad fighting (Kitch, 2001).

Women were seen as more sensual during this time. The desired physique was once again shapely. The physical ideal was slim but curvy with a defined waist (Banner, 1983; Grogan, 1999; Kitch, 2001). Fashions favored tailor-made suits - skirts and jackets - slimming and form fitting. Skirts were longer and shoulder pads were used to create broader shoulders. The hourglass shape was back for women.

Film stars like Greta Garbo and Rosalind Russell represented the sociocultural ideal beauty of the 1930 s and 1940 s. Another popular film star, Betty Grable, was the first pin up poster in America. Movies became popular because they took the country's mind off the depression and then the war (Banner, 1983). Magazines continued to engage the subscribers with their sociocultural ideal illustrated on the covers (Kitch, 2001). On the whole, 
magazines and movies became major communicators of the American ideal beauty (Banner, 1983).

1945-1965

The post war years brought with them the "Baby Boomers". The country was in a period of economic expansion. There were fewer jobs for women once the men returned from war. Women generally opted to marry and settled down to become "happy housewives" while the men supported their families (HesseBiber, 1996). Consumerism shifted to mothers and adolescents (Hesse-Biber, 1996). It was once again a time to celebrate youth.

The hourglass figure was still the physical ideal but more voluptuous. The large bosom and wide hips with a narrow waist much like a modified Victorian figure only without all the clothes (Banner, 1983; Hesse-Biber, 1996). Women were wearing girdles again as this helped showcase the curvaceous figure. Blonde hair became a big hit in the 1950 s as it symbolized femininity, sensuality, and unintelligence (Banner, 1983). Women were dying their hair in the belief that "blondes have more fun" (Banner, 1983). In fashion, clothes varied, but skirts lengthened to midcalf length and became fuller. Notwithstanding, leisure clothes revealed more of the body. Midriff tops and short shorts came into vogue. Twopiece swimming suits became common. There was a clear distinction between formal and leisure clothing.

The introduction of the Barbie ${ }^{\mathrm{TM}}$ doll occurred around 1959. She was an instant success with girls. Even four-years-old girls owned a Barbie doll. She 
reflected the perfect figure (Maine, 2000; Mann, 1994). Barbie was the perfect representation of the American sociocultural ideal for girls.

Television became a permanent fixture in households during the $1950 \mathrm{~s}$ and increased in popularity in the 1960s. Magazines and movies were still impacting the country with their messages and conveying the American standard of beauty. Hollywood provided a well-endowed new ideal image in Marilyn Monroe and later in Jayne Mansfield. In particular, Marilyn Monroe typified the era's major beauty ideal with her sexuality and childlike innocence (Banner, 1983). Magazines, the most influential medium of the time, still were using cover illustrations to embody the American ideal.

1965-1985

The late 1960s were a time of unrest, and the Civil Rights movement was going strong. Minorities and young people became disenchanted with the establishment in America, and there were demonstrations and riots. Women were burning bras and demanding equal rights. The contraceptive pill played a role in women gaining control over their bodies. Many returned to the workforce. Equally important, young people demanded freedom to explore and make their own decision about life. This was the turning point for American youth and their culture.

Twiggy made a big splash on the modeling scene in the mid 1960s with her twiglike body, short hair and mini skirt. She weighed 91 pounds and was $5^{\prime} 7^{\prime \prime}$ (Maine, 2000). Twiggy embodied youth and the fresh young look appealed to young women. The American ideal changed overnight. Once again, the ideal 
body was thin, straight, flat breasted, and long legged (Grogan, 1999; Maine, 2000). Thin was in. This physical ideal continued over the next 20 years. Clothes were minimal due to Twiggy's influence. Skimpy was in - mini skirts, halters, and dresses - showcasing the body. Thereafter, hemlines moved up and down depending on the fashion. After the 1960s, everyday clothes covered up the body. The body still was exposed more than in the past. Leisure clothes did not change much except for the bikini and popularity of jeans in the 1960 s. There was still a distinction between everyday clothes and leisure clothes.

On the health front, strict dieting was constant as the thin figure was difficult to sustain for many women. Starvation or liquid diets reigned. The idea was to lose weight fast and then keep it off. This concept continued through the 1980 s.

Media began gaining power during this time with the popularity of television and the explosion of new technology. Hollywood became less influential as portraying the American ideal. Television solidified its dominance of the media with the advent of cable stations during the 1970s (Strasburger \& Wilson, 2002). Because it had an immediate visual audience, advertisers moved to television (Banner, 1983). Movie videos became popular over this period. Unfortunately, magazines lost advertisers and had to change their format, such as using photographs instead of illustrations as well as becoming more specialized (Kitch, 2001). Basically, they had to regroup. With advances in technology, media were broadening their scope. 
1985- present

Americans currently live in a quick fix as well as a self-obsessed society (Grogan, 1999; Kitch, 2001). We work to spend money. In most households, there are two adults working outside the home. Women are getting better paying jobs, being selected as CEOs, and starting their own businesses (Wolf, 2002). Consumerism has increased exponentially. Children helped create the pop culture, in which they must own brand names and certain products. Parents usually accommodate them. At the same time, most members of society have become less active than in the past. As a result, two thirds of Americans are currently overweight (Centers for Disease Control and Prevention, 2006). Nevertheless, families are hurried, each with their own life that they rarely sit down together to eat. Actually, children spend more time with media or their friends than family (Strasburger \& Wilson, 2002). Above all, youth are still the focal group in society.

Since the mid '60s, thin has been the American physical ideal. That has not changed. During the late 1980s, muscle sculpting became part of the workout regime in order to achieve a tight, defined thin body (Grogan, 1999). That trend passed, but the thin ideal remained. Cosmetic surgery, like breast augmentation, has become the quick fix for anything that is flawed. Currently, girls request breast augmentation more than any other gift for high school graduation (Davis, 2004). Exercising as well as a healthy diet made a comeback around the 1990s (Maine, 2000). Today, most females are trying to lose weight in a healthier fashion. In contrast, obese girls are opting for gastric bypass as a 
quick fix to losing weight. At the same time, eating disorders gained public scrutiny around the 1980 s and continue to be a problem for some females (Grogan, 1999). In any event, more females are feeling the pressure to be thin (Garner, 1997; Grogan, 1999; Maine, 2000).

Clothes have changed towards a more casual style. Jeans became a permanent part of young women's attire. The '80s trend was workout clothes as well as tight clothes. The '90s saw holes in jeans and tight tops. Victoria's Secret became the place to buy undergarments. The thong made its debut for underwear and swimsuits. Tattooing and piercing were fashion statements (Maine, 2000). There are now plus size clothes sold in most stores and catalogs. At the start of the millennium, clothes were minimal again like the late ' 60 s. Currently, no distinctions can be made between everyday and casual clothes. Media have increased their power over this 20-year period (Strasburger \& Wilson, 2002). We now live in a celebrity culture. Celebrities in all the mediums have gained popularity. Models like Kate Moss and the heroin chics reflect the ideal body image. The Olsen twins and the "Friends" cast from television also mirror this ideal. In fact, television is still the most popular medium, and is viewed now even more than in the past. Despite the dominance of television, magazines continue to have a large readership today (Kitch, 2001). The print industry found a large readership with teenagers and created what they termed "teenzines", which has become a multibillion-dollar business. There is also the Internet that has become part of the current culture. Game and music videos also are popular with the youth today (Strasburger \& Wilson, 2002). Media have 
deluged us with many forms of communication. They all communicate the cultural ideal in some form or fashion.

Since the 1960s, television and magazines have become prominent communicators of the sociocultural ideal (Grogan, 1999; Maine, 2000; Thompson et al., 1999). This 20 -year period has seen many advances in technology, yet the physical ideal remained the same. The only difference today is that there are two models of thin - naturally thin and thin with enhanced breasts.

In summary, this historical review of the American sociocultural ideal has shown that trends were set by the times and beliefs of culture and society. In the 1800 s, the Victorian woman displayed her beauty through her curvaceous physical figure, an hourglass silhouette. It was her beauty that enabled her to catch the most prosperous man for a husband at the time. This was considered her duty. At the turn of the twentieth century, the silhouette was more voluptuous and women were still showcasing their beauty through the silhouette. Women were enjoying through recreation this beautification process to catch a prosperous husband. Over a twenty-year period, thin was the ideal and fewer clothes were worn but the body was still covered from the neck to knees. The flapper represented carefree young women who enjoyed flaunting their bodies. Their beauty regime offered internal pleasure for women before having to get serious and search for a future husband. After the flapper period, the curvaceous bodies returned where clothes were more provocative and showcased their bodies from the 1930 s through early 1960 s. Beautifying was both an internal and external need as women wanted to feel good about 
themselves as well as entice the men. From 1965 onward, the youth culture gained status and thinness became the standard of beauty. With this beauty management, women strived to feel good about themselves by looking good especially by being thin. Throughout the last two centuries, the thin body image embodied youth, whereas the curvaceous body personified the mature woman. Whenever the sociocultural ideal body image changed, women tried to follow the status quo. Whatever the sociocultural ideal, women wanted to model it.

\section{Adolescents}

Females have been the prime focus of body image research, because they have been self-conscious of their bodies since childhood (Brumberg, 1997; Mann, 1994). In particular, female adolescents worry more about body image than any other age group (Grogan, 1999; Maine, 2000; Mann, 1994). This developmental phase is one of change, self-consciousness, and identity search.

\section{Development}

As it can last over a decade, adolescence begins as early as 10 and sometimes lingers to age 30 . The essence of adolescence is fluctuation and change. Developmental transitions are notable in the physical, psychological, and social domains. For instance, puberty triggers change as adolescents' physical body and endocrine system matures (Berk, 2004). For many girls, their bodies become unfamiliar as they make all these adaptations. Physical, psychological, and social changes generally occur during early (10-13 years) and middle (14-17 years) adolescence, while late (17+ years) adolescence brings psychological and social changes (Berk, 2004). 
Late adolescence is a time to question identity by asking, "Who am I?" and "What do I want?" Adolescents explore their identity through trial and error during this phase of their life. In fact, adolescents' emergence of autonomy brings forth a clearer sense of their self as well as independence from their family and friends (Berk, 2004). In particular, adolescents make more decisions without relying on others. Cognitive maturity helps initiate this process. Overall, adolescents have higher reasoning levels in their problem solving, perspective taking, and abstract thinking at this stage than previously in their development (Berk, 2004).

Relationships are an essential part of female adolescents' lives. Because young women spend more time with peers, they are as important as family during late adolescence. This also is a time when young women narrow their relationships down to one or two close friends and usually a dating partner (Berk, 2004). Female adolescents rely on these select peers to be honest and forthright, especially with respect to their concerns about body image (Berk, 2004; Nichter \& Vuckovic, 1994; Pipher, 1994).

\section{Body Image}

Young women in late adolescence have acquired beliefs and values of their culture and society through family, friends, peers, institutions, and media during their development. Which beliefs and values these young women acquire depend on their individual relevance, and the particular beliefs and values have been filtered by the personal beliefs and values of each. Adolescents, however, are impressionable. In fact, the sociocultural preference for females to be thin is 
currently prevalent among adolescents (Brooks-Gunn \& Reiter, 1990; Grogan, 1999; Levine \& Smolak, 2002). Being thin is associated with social acceptance, while being fat, social rejection (Grogan, 1999; Jackson, 1992; Schwartz \& Brownell, 2002). Female adolescents have a strong need for peer acceptance and want to belong (Jackson, 1992; Nichter \& Vuckovic, 1994; Pipher, 1994). These are a few reasons why body image concerns are a top priority for female adolescents (Grogan, 1999; Harter, 1990; Levine \& Smolak, 2002; Pipher, 1994). "How I look" is associated with identity and psychological and social development (Berk, 2004; Pipher, 1994). As Joan Brumberg (1997, pg. 97) pointed out, "young women believe that the body is the ultimate expression of the self."

Most young women are self-critical of their body image, and many have a poor body image (Brooks-Gunn \& Reiter, 1990; Grogan, 1999; Harter, 1990; Levine \& Smolak, 2002). Even though most young women are familiar with their physical maturity, they still overestimate their body shape and size (Brumberg, 1997; Grogan, 1999; Levine \& Smolak, 2002). Young women in late adolescence must become reacquainted with their bodies, and somatic changes continue to be an important concern during this phase in life.

\section{Experiential Factors}

The sociocultural perspective introduced the idea that sociocultural concepts become integrated into female adolescents' thinking and behavior. They have acquired from family, friends, and media the sociocultural standards of their social environment. According to the sociocultural theory, these experiential factors of family, peers, and media influence adolescents' 
perceptions of their current and ideal body images. Three types of experiential factors are especially important toward influencing the perceptions and attitudes of body image: direct, vicarious, and the interaction of the direct and vicarious experiences.

In the current study, research in these three areas was explored. First, this study examined direct experiences of the dynamics of family and peers with female adolescents to determine what impact or whether they impacted adolescents' body image. Second, it explored vicarious experiences of the print and electronic media. Research of these vicarious experiences scrutinized the influence of media on female adolescents' body image. Finally, the interaction of experiential factors focused on the impact of the interactions of direct and vicarious experiences.

There are a few qualifiers in the studies presented herein. The majority of studies were self-reported, especially height and weight. Because females are the focus of this study, no results were given for males in the gender studies. Further, the majority of the body image research have a predominately White ethnic group. The White population generally represented over $70 \%$ of the participants in the various studies. Therefore, no distinctions were made with ethnicity, except in cases of comparison groups, like Black and White, and larger ethnic distributions. There also were some studies presented with an Australian population; it has been established that American and Australian women have similar patterns of body image perceptions (Grogan, 1999). 


\section{Direct Experiences}

Female adolescents are exposed from birth to the cultural and social values of their family and community. These social relationships help her to coconstruct values and beliefs throughout the formative years. Within this sociocultural environment, a female adolescent receives both verbal and nonverbal messages from her family and peers about the female ideal body image and her own body image. As a result, family and peers generally evaluate the adolescent's body image against the thin ideal body image (Nichter \& Vuckovic, 1994; Johnson, Roberts, \& Worell, 1999; Pipher, 1994). Family

The family teaches values, beliefs, and customs throughout childhood and adolescence. Typically, parents are the center of identification and transmit their messages to the children. Children's perception of body image is acquired through family members by observation and feedback of others' beliefs. Within the family, female adolescents interact daily with their families and are exposed to comments about their own physical development. The family transmits messages to the adolescent about how her body image stacks up to the sociocultural ideal (Johnson et al., 1999; Kearney-Cooke, 2002; Pipher, 1994; Thompson et al., 1999). How do the father, mother, and sibling(s) influence the self-perception of female adolescents' body image?

Parents. The majority of the body image studies involving parental influence focused on the mother's role (Kaplan, 1997; Thomas, 1989; StriegelMoore \& Cachelin, 1999; Wertheim, Paxton, Schutz, \& Muir, 1997). These 
studies suggest that parents send messages about her body image through modeling, feedback, and/or instruction to their daughters. Implicitly or explicitly, such messages impart the sociocultural ideal to female adolescents.

Levinson, Powell, and Steelman (1986) investigated the connection between parents' assessment of their daughter's weight and the daughter's perception of her weight. This study was conducted in 1970 with over 3178 females, $84 \%$ White and $16 \%$ Black, between the ages of 12 to 17 years. Data were obtained from Cycle III of the National Health Examination Survey. A physician who examined and interviewed participants as well as interviewed one parent conducted the study. The study showed that adolescents were discontented with their weight, thus confirming the notion that most adolescents are self-critical of their body images. The investigators found a strong relationship between the parents' assessment of the daughter's body image and the adolescents' perception of their body image. The researchers pointed out that even though there was a correlation, cause and effect were debatable. The study demonstrated that the parents' views of their daughter's body image affected how daughter's perceived their body image.

Female adolescents were aware of when their parents thought daughters should have a smaller shape and size. Dunkley, Wertheim, and Paxton (2001) conducted a study on sociocultural influences of body image dissatisfaction with 577 Australian adolescent girls ranging in age from 13 to 17 years. Self-reported questionnaires were used in this study. The researchers concluded that daughters thought their parents ( $41 \%$ fathers and $45 \%$ mothers) wanted them to 
be thinner than they actually were. Still, parents exerted the least amount of pressure to be thin when compared to other sociocultural agents. Daughters believed that their parents actually influenced their body image and dieting behavior. The results of this study supported the notion that those girls who showed the most body dissatisfaction and dietary restraint lived in a subculture that supported the thin ideal and encouraged dieting (Dunkley et al., 2001).

Levine and Smolak (2002) stated that direct comments about weight, body, and eating were potent sources of parental influence. In fact, parents tended to be more critical of their daughters' appearance once they reached adolescence. Striegel-Moore and Kearney-Cooke (1994) conducted a survey study of parents' evaluations of children's body image, eating habits and exercise behaviors, and parental efforts to influence their children's body image. In this study, 1276 adults who had at least one child between the ages of 2 to 16 years were surveyed. In particular, the adults with more than one child were asked to randomly select one child as the "target" child. The data indicated that the parents were basically satisfied with their target child's body image, eating habits, and exercise behaviors. Nevertheless, there was a strong relationship between parents who were concerned about their weight and their children's weight. These parents were more likely to instruct their daughters or model for them how to diet and lose weight. Equally important, the researchers found that parents became more critical about the children's body image as the children age. The findings suggested that this pattern helped to perpetuate body dissatisfaction among females as the messages of criticism escalated during adolescence. 
In a similar study, Kaplan (1997) interviewed 24 diverse young women ranging in age from 19 to 35 years with a mean age of 22 years. She explored their perceptions of their adolescent experiences. Kaplan discovered that parents had begun focusing on their daughters' appearance in adolescence. Some had started pressuring their daughters to be more feminine and conveying messages about the desired female body image. Negative comments by parents about their adolescents' body image were recalled as hurtful. In contrast, parents' satisfaction with their daughter's body image during adolescence was reflected by their daughter's own satisfaction with her body image. Jaffe and Lutter (1995) examined factors influencing body image with 152 female adolescents ranging in age from 11 to 17 years. The ethnic composition was 43.4\% White, 16.4 \% Black, 5.9\% American Indian, 7.2\% Hispanic, 2\% Asian, $6.6 \%$ Biracial, and $18.5 \%$ other. The surveyors determined that daughters' receiving positive comments from parents on appearance and achievements had a more positive body image than daughters receiving negative comments from their parents. In fact, they suggested that parents' comments had an impact on girls' body image and achievements. In another study, Thomas (1989) examined 102 Black women under 35 years old from Washington, D.C. She rated their perception of significant others with parents as one category. In this survey study, Thomas reported that parents' perceptions $(r=.64, p<.0001)$ were strongly related to the women's body image satisfaction and self-esteem. This study indicated the women's perceptions reflected their parents. 
As suggested in the preceding studies, Kearney-Cooke (2002) surmised from the body image research that parents' reactions towards children contributed to the development of their body image. Likewise, Garner (1997) surveyed 3452 women ranging in age of 13 to 90 years for the Psychology Today's 1997 Body Image Survey. Garner discovered that $31 \%$ of the women reported their mothers and $23 \%$ their fathers as having influenced their body image.

Mothers. A daughter is most likely to identify with her mother, and some even imitate their mother's actions. As the mother is generally the caretaker, daughters spend more time around her than the father. Some daughters acquire some of their mother's attitudes and behaviors especially how she feels towards her body. Kearnery-Cooke (2002, p. 102) claimed, “Today's young women are the first generation to be raised by mothers who typically reject their own bodies."

How do mothers influence their daughter's self-perception of body image? The Teen Lifestyle Project, a three-year longitudinal study, probed adolescents about their body image, dieting, and smoking. Nichter and Vuckovic (1994) interviewed 253 female adolescents in the eighth and ninth grades in the southwestern United States. They determined that the majority of the girls were within normal height and weight ranges. The ethnographic data indicated that familial dieting especially by the mothers significantly influenced these adolescents. In particular, Nichter and Vuckovic (1994) found that $30 \%$ of the participants had been told by their mothers that they needed to lose weight even though less than $5 \%$ were overweight. The researchers suggested that the 
mother's advice reflected the daughter's own worry about her body image. Sixtyeight percent of the participants reported that their mothers regularly dieted and some would diet along with their mother.

Hesse-Biber (1996) claimed that the mother often initiated the daughter into the rites of losing weight. In a survey study, Sanftner, Crowther, Crawford, \& Watts (1996) questioned 382 diverse female adolescents ranging in age from 9 to 15 and their mothers to find out whether there was a relationship between mothers and daughters' habits. There was a relationship between mothers and daughters' weight preoccupation and eating psychopathology at least after the daughters went through puberty. Sanftner et al. (1996) advised caution when interpreting their findings because mothers and daughters might have used similar tactics to cope with the cultural standards. If so, mothers' really did not exert much influence.

Rieves and Cash (1996) surveyed 152 college women about teasing and criticism of their body image. Thirty percent of the participants reported that their mothers had criticized their physical appearance. The study revealed that such criticisms affected the daughters' body image. The experimenters suggested that teasing might have long lasting effects.

Thompson et al. (1999) stated that the mothers' influence of female adolescents who were dissatisfied with their own bodies, encouraged their daughters to diet. In extreme cases of dieting and body dissatisfaction, the mothers' behaviors predicted the daughters' behaviors. These extreme mothers might be unaware of modeling such behaviors to their daughters. Mothers who 
had an eating disorder were significantly more likely to have daughters with the same disorder (Striegel-Moore \& Cachelin, 1999; Thompson et al., 1999).

Fathers. The role of the father varies widely in America. Some fathers have close and nurturing relationships with their daughters while others barely interact with them. According to Mary Pipher (1994, p. 117), "The main job of fathers is to teach their children to fit into broader society."

Kearney-Cooke (2002) suggested that the father's reaction to his daughter's changing body during adolescence played an important role in shaping her attitude about her body. She stated, "The father's views on the 'ideal' body for women, his attitudes toward women's bodies, his reactions to his daughter's changing body, and his comments about her maturity have an impact on her body schema and are likely to influence her body image development in either positive or negative ways" (Kearney-Cooke, 2002, p. 103). In a fatherdaughter relationship study, Mary Pipher (1994) reported that fathers could be supportive or unsupportive in these relationships. Supportive fathers did not criticize daughters' looks or physique. Fathers only tried to encourage their daughters to be mentally and physically healthy. Whereas nonsupportive fathers who emphasized the sociocultural standards, could damage the daughters' selfconfidence. The unsupportive fathers tended to be sexist and wanted their daughters to learn to please men. These fathers wanted their daughters to be accepted by society and encouraged them to lose weight in order to fit in. In essence, the unsupportive fathers influenced daughters to believe that their only 
value was physical attractiveness. Pipher (1994) suggested that daughters of unsupportive fathers would be more likely to internalize the sociocultural ideal.

In an ethnographic study, Kaplan (1997) examined the transitional period from preadolescence to adolescence of 24 undergraduate women ranging in age from 19-35. The author concluded that fathers pressured their daughters to lose weight, and conveyed messages about the sociocultural ideal through his remarks about other women around them. The young women reported feeling pressured to diet and worried about their appearance.

In a survey of midadolescent girls, fathers generally were reported as noninfluential in daughters concern of their body image (Wertheim et al., 1997). This study of $\mathbf{3 0}$ fifteen-year-old adolescent girls in Australia surveyed body concerns. In a rating of "pressure to be thin", fathers ranked ninth among ten listed sociocultural influences. Negative comments about their daughters' body image had some effect, but it was not long lasting. Rieves and Cash (1996) found that $24 \%$ of the 152 college women they surveyed reported that their fathers had criticized their body image. Unlike Wertheim et al., this study determined that the criticism could be associated with long-term body dissatisfaction. Finally, sexual abuse was found to contribute to adolescents' body dissatisfaction, weight concerns, eating disorders, and low self-esteem (Levine \& Smolak, 2002; Pipher, 1994).

Siblings. Relationships with siblings are complex during adolescence. Where the female adolescent fits within the family structure is an important element as well as the siblings' dynamics. Rieves and Cash (1996) examined 
siblings' social comparison with adolescents' body image attitudes and behaviors. They discovered that a more attractive sibling tended to perpetuate unfavorable self-evaluations of body image, whereas a less attractive sibling tended to enhance them. The research about siblings who had a better body image than their sister was inconclusive regarding whether it affected her personal body image evaluation. However, the perceived effects of a siblings' positive body image were significantly related to the sister's placing importance on her body image.

Sibling teasing was also explored in the Rieves and Cash (1996) study. Teasing a girl about her body image or some part of her body made lasting impressions. Brothers were found to be the worse culprits. Seventy-nine percent of the respondents cited their brothers as the main perpetrator. Thirty-six percent of the participants also identified sisters as doing their share of teasing. Siblings who teased their sister about her body promoted dissatisfaction (Levine \& Smolak, 2002; Rieves \& Cash, 1996).

Peers

Peers are the most important and influential social group for late adolescent females. Since female adolescents are immersed in American pop culture, they are repeatedly exposed to the thin sociocultural ideal body image. Peers discuss the sociocultural ideal regularly, and they often measure themselves against the standard (Nichter \& Vuckovic, 1994; Pipher, 1994). During these discussions, females generally receive feedback about their body image from peers. They also socially compare themselves to their peers. 
Ultimately, their body images are affected by these interactions, and due to their intimacy, dating partners are even more influential. Adolescents tend to believe that peers provide a more honest feedback about their body image than parents (Nichter \& Vuckovic, 1994; Pipher, 1994). As adolescents work through their struggles with body image, peers of both sexes are the ones female adolescents rely on for feedback and support.

Adolescents, especially those who are dissatisfied with their body image, often compare themselves to others. In Jaffee and Lutter's (1995) study on factors influencing body image, they found that $52 \%$ of the adolescents who have a low body image were likely to compare themselves to peers with "perfect" bodies. These authors pointed out that "perfect" body statements were selected more often than the same phrases the words with "perfect" omitted. The concept of a "perfect" body was important to the girls in this study as it implied the thin ideal body image. Those with a poor body image reported that this image was influenced by feedback from peers.

"I'm so fat!" was a common pronouncement among adolescents. In Nichter and Vuckovic's Teen Lifestyle Project (1994), the authors found that by the time a girl reached adolescence she was probably a competent user of the expression whether or not she actually practiced weight control. Fat talk described a wide range of feelings regarding no control in female adolescents' lives. "I'm so fat" was a call for support from peers for affirmation that one was not fat. This expression had many different contexts and peers were able to properly interpret them. Generally, girls who proclaimed "I'm so fat" were not 
overweight, but were unsatisfied with their body image. In fact, overweight girls tended not to use this expression, as it would call attention. The "I'm so fat" expression informed the girls about what others thought about them, ergo body image.

Girls liked to hear others tell them that they were not fat. Using the expression before a meal indicated that one knew she should be on a diet even though she was not overweight. Adolescents also used this expression to fit in with peers. Group fat talk allowed members to obtain affirmation that they looked good (Nichter \& Vuckovic, 1994). The group dieted together to support each other, if one felt fat then the others figured they needed to lose weight as well. Incidentally, dieting was short-term for this group - only a couple of hours or a day or, at most, a week.

Nichter and Vuckovic (1994) learned that $84 \%$ of the interviewed 523 female adolescents did not consider thinness to be a criterion for friendship except with the popular group. Basically, females who engaged in fat talk were presenting themselves to others as someone responsible about her body image. Female adolescents' experiences of the "I'm so fat" discourse and body dissatisfaction allowed them to reflect rather than deviate from their peers. According to Nichter and Vuckovic (1994), adolescents were attempting to reproduce the sociocultural ideal and negotiate peer acceptance through their "I'm so fat" discourse.

Peers often tease one another. Unfortunately, this behavior has become more mean spirited with today's adolescents as the teasers have become more 
graphic and the verbiage outrageous. In Rieves and Cash (1996) study, friends and peers were the second worse group to tease females about their body image. Sixty-two percent of peers and forty-seven percent of friends were reported teasing the participants in adolescence. Males were most likely to tease about weight and shape. Teasing by peers had a lasting effect on a female's body image. It also was associated with more dieting.

Friends. Friends have more personal knowledge about one another than family. Friends have similar likes and dislikes. Female friends spend endless amounts of time talking. One popular topic is body image. Friends tend to have similar perspectives about body image concerns, sociocultural ideal body image, and dietary habits (Grogan, 1999; Nichter \& Vuckovic, 1994). The Paxton, Schutz, Wertheim, and Muir (1999) study of interpersonal influences concluded that female adolescents within the friendship group were alike in concerns about body image and dieting behaviors. The 523 girls from Australia also reported talking about body image concerns and dieting. Paxton and colleagues (1999) confirmed that perceived friends' attitudes and behaviors toward weight and diet predicted a female adolescent's own body image concerns and dieting behavior - a mirror image.

Thomas's (1989) body image study revealed that close male friends' perceptions about Black women's body image and the women's own body image was a strong positive correlation $(r=.56)$. Close female friends' perceptions also were found to have a direct correlation with the women's body image $(r=.52)$. 
Thomas (1989) noted that the body image assessments were influenced by the women's self-report of the perception of the significant others.

Dating Partners. Dating partners are more vulnerable to one another's opinions than those found in any other type of peer relationship. Most often, relationships are based upon perceptions of physical appearance and personality (Berk, 2004; Tantleff-Dunn \& Gokee, 2002; Thompson et al., 1999). In fact, males are more likely to place emphasis on physical attractiveness when selecting a mate than females (Tantleff-Dunn \& Gokee, 2002; Thompson et al., 1999). Dating partners are considered one of the most important interpersonal factors in determining female's body image (Nichter \& Vuckovic, 1994;TantleffDunn \& Gokee, 2002; Thompson et al., 1999).

In Garner's Body Image Survey (1997), 40\% of the 3452 respondents stated that partners' opinions about women's body image were very important to their own body image. Garner explained that there was a connection between one's body image perceptions and the way one perceived how others feel about it. Mark and Crowther (1997, as cited in Thompson et al., 1999) studied the link between relationship satisfaction and body image attitude among young, unmarried women. They reported that the less satisfaction in the relationship the more a woman was dissatisfied with her body and vice versa.

In a study by Rieves and Cash (1999, as cited in Thompson et al., 1999), the Physical Appearance in Intimate Relationships Scale (PAIRS) measured female adolescent's perceptions of her dating partner's attitudes and behavior towards her appearance. PAIRS scores were negatively correlated with body 
dissatisfaction and less relational and sexual satisfaction for both partners. In another study, Thomas (1989) found the same relationship between male's perceptions of his female partner's body image and her own body image, at least among Black women. In essence, a male's relationship satisfaction was significantly related to satisfaction with his partner's body image (Tantleff-Dunn \& Gokee, 2002; Thomas, 1989; Thompson et al., 1999).

Some sexual partners actually monitor mates' weight and pressure their mates to lose weight (Grogan, 1999). In Gimlin's bodywork research (2002), women reported in interviews that their mates told them that their bodies did not meet cultural standards; some even made fat comments. Gimlin found these negative comments affected the women's body image. The more often the negative comments were made, the more the women's body image deteriorated. In fact, some women took aerobics classes to work out the flaws in their bodies. The women in Gimlin's (2002) bodywork study changed their behavior to please their partners and improve their self both mentally and physically.

Fear of losing one's dating partner to someone with a better body can put undue pressure on female adolescents to maintain a good body image (Ferron, 2000). Murray, Touyz, and Beumont (1995) explored through interviews the interpersonal influences on body image and weight by comparing females with eating disorders to female and male control groups. There were 80 women with eating disorders and 82 females and 69 males in the control groups. The interviewers discovered that $48 \%$ of the participants claimed that the opposite sex affected their eating, exercise, and body satisfaction with no difference 
between comparison groups. The opposite sex influenced the others' perceptions, attitudes, and behavior according to the research.

On the whole, the literature supports the notion that family and peers influence female adolescents' body image. Two behaviors are especially important - teasing and negative parental feedback. Teasing was one area where the family dynamics were unequivocally influential in the daughter/sister's body image. Negative parental feedback about the daughter's body image was another. Researchers found that criticism and/or teasing have both short and long term effects on a young woman's perceptions of her body image (Levine \& Smolak, 2002; Rieves \& Cash, 1996). In Garner's Body Image Survey (1997), teasing during adolescence had an unforgettable effect on women's feelings about their bodies. Women said that the negative result can last for decades no matter what shape they were (Garner, 1997). Undeniably, research has found a connection between teasing and body image problems (Garner, 1997; Jaffee \& Lutter, 1995; Levine \& Smolak, 2002; Rieves \& Cash, 1996; Tantleff-Dunn \& Gokee, 2002). A similar effect was also found with peer teasing. With regards to influence, Harter (1990) found that peers were more influential than family in adolescence and early adulthood. Research also has shown that criticism and social comparison with peers profoundly affect the body image of females (Jaffe \& Lutter, 1995; Nichter \& Vuckovic, 1994; Rieves \& Cash, 1996; Thomas, 1989). Through social comparisons, female adolescents were generally dissatisfied with their body image when comparing themselves to peers who had the thin ideal body image. Dating partners who criticized their girlfriends impacted the young 
women's body image. Basically, criticism and teasing were the two major traits influencing female adolescents' body image with family and peers. Through social comparison, female peers who were thin also impacted female adolescents. When it came to direct experiences, female adolescents receive direct and immediate feedback from family and peers, thereby having an instantaneous effect on their perceptions and attitudes towards their body image. There was no determination whether the effects of feedback were short or long term, however, teasing may have a long term effect.

\section{Vicanious Experiences}

\section{Media}

Media are powerful vicarious conveyors of sociocultural messages. Their messages are indirect, subtle, and cumulative (Strasburger, 1995; Strasburger \& Wilson, 2002). Today's youth have always had media as part of their lives. They wake up to it, use it throughout the day, and go to bed with it. Young people spend about one third to one half their waking hours with some form of media (Strasburger \& Wilson, 2002). They have had many years of viewing media. Clearly, media messages are being conveyed to adolescents.

Media play a major role in communicating the sociocultural ideal body image. It is displayed on a daily basis. And today's adolescents covet celebrities more than in past history (Brumberg, 1997; Grogan, 1999; Kitch, 2001).

According to Signorelli (1997), $69 \%$ of the girls surveyed even wanted to look like a character on television. Most female celebrities model the thin ideal; be it acting, singing and dancing, or fashion modeling. Adolescents receive the 
message from media that celebrities are happy, liked, in control, and have their selection of mates (Grogan, 1999; Tiggemann, 2002; Thompson et al., 1999). Undeniably, media glorify the thin ideal message. By the time adolescents are 18 , visual images of the thin ideal have been seen thousands maybe even millions of times, as television produces thirty images per second (Cole, Browning, \& Schroeder, 2003). Female adolescents have been deluged with the media ideal body image over time. With this prevalent visual icon, female adolescents may internalize the media's message and adapt it. It is the standard in which females may measure their current body image against. This distinction between the current and media ideal body images, in turn, generally influences adolescents' attitudes and behaviors.

Television, magazines, music videos and other mediums display women with perfect bodies and vicariously pressure female adolescents to conform (Strasburger \& Wilson, 2002). Media research has determined television programming, magazines, and music videos influence female adolescents; these mediums are the young women's preferred form of entertainment in late adolescence (Signorelli, 1997; Strasburger \& Wilson, 2002).

Media in general. Media have been identified most often as the source of the thin ideal for female adolescents (Tiggemann, Gardiner, \& Slater, 2000; Strasburger, 1995). In the Tiggemann et al. (2000) body concern study, 67 sixteen-year-olds from Australia were interviewed and identified various sociocultural influences as determinants of their desire to be thin such as media, fashion industry, and pressure from peers. Tiggemann et al. (2000) concluded 
that media was the most frequent and strongest determinant for these adolescent girls. They reported that the constant bombardment by media of thin women led them to believe that thinness was the sociocultural norm. This group realized that the thin ideal was unrealistic, but striving to be thin was still important to them. The girls also reported that they would be more confident when they were thin. However, a notable finding was that the desire to be thinner did not necessarily mean that girls were dissatisfied with their body image. Basically, this study revealed that media influenced the standard of the thin ideal and adolescents desire to be thin; however, the discrepancy between the sociocultural ideal and their current body image did not necessarily lead to a negative body image attitude.

In Garner's survey study (1997), the author investigated the growing gap between current and preferred body images. Garner (1997) found that $43 \%$ of the women reported that the "very thin or muscular models" portrayed by media made them feel insecure about their weight. Moreover, $48 \%$ of the women indicated that the very thin models made them want to lose weight, so they would have the same body shape. Garner (1997) discovered that media's impact was somewhat selective, because it most strongly influenced those who were dissatisfied with their shape and weight. In fact, $56 \%$ of the women reported being dissatisfied with their body image; young women especially were afflicted. When Garner compared his three body image survey studies, women from the 1997 study were more dissatisfied with their body image. In particular, results from the 1972 survey reported that $25 \%$ of the women were dissatisfied with their 
bodies, while the 1985 survey reported $38 \%$, and the 1997 survey reported $56 \%$.

The author concluded that more women were disenchanted with their body image than ever before. According to Garner (1997, p. 17), "thinness has become the preeminent yardstick for success or failure, a constant against which every women can be measured."

Some media studies applied the social comparison theory to investigate media's influence on body image attitudes and behaviors of women. Jaffe and Lutter (1995) explored factors influencing body image with 152 girls aged 11 to 17 years. Twenty-six of those 152 girls had a low body image. The survey found that girls with low body images compared themselves to the media ideal, whereas girls with high body images did so less often. The same results were found when the girls were assessed for social comparison with their peers.

In another social comparison study, Posavac, Posavac, and Posavac (1998) conducted three experiments to determine how media exposure affected body concerns with 370 college females between 18 to 25 years of age in a university setting. Posavac et al. (1998) learned that exposure to the media ideal had the potential to increase weight concern among most young women. The study suggested that the effect of media exposure on females' body concerns derive from social comparison. Furthermore, women who were satisfied with their body image did not change their views when exposed to the media ideal. The researchers noted that slides of attractive college women presented during the study had little effect on participants' body concerns, but the experts were unable to explain why this occurred. The study suggested that media exposure 
of the thin ideal had an effect on body concerns from a social comparison perspective among young women who were dissatisfied with their body image.

Another comparative study investigated the influence of media images on adolescents' body image and the thin ideal endorsement (Botta, 1999). The survey was administered to 214 high school girls who had an average age of 15.19 years from the midwest. The ethnic composition was $67.8 \%$ White, $15.4 \%$ Black, $11.2 \%$ other, and $5.6 \%$ unreported. Botta (1999) advanced that media impacted body image disturbance directly through body image processing and indirectly by enticing the adolescents to endorse the thin ideal. In other words, the adolescents' body image comparisons with celebrities were significantly related to body dissatisfaction.

In a survey study, Durkin and Paxton (2002) studied changes in body image satisfaction and psychological well being for female adolescents when exposed to the media ideal body image. The participants were 116 grade 7 (average age 12.9 years) and 125 grade 10 (average age 15.5 years) girls from Australia. The experimental group was exposed via fashion advertisements to the media ideal body image. The study determined that there was reduction in body satisfaction and mood following exposure to the media ideal. With the control group, the girls viewed fashion accessories advertisements such as make-up, jewelry, and shoes. The outcome from this group was that these images had no influence on body satisfaction. In essence, the authors established that media influence was through the models' images rather than the advertised products. Durkin and Paxton (2002) also found that exposure to the 
media ideal body image had a short-term negative influence on state body satisfaction and mood. Further, the media ideal body image was more influential with the older cohorts than the younger ones. The investigators indicated the importance of individual differences in short-term influence of viewing the media ideal. This inquiry supported the notion that the influence of media on adolescents' body image was temporary.

A few studies have found that the media ideal does not influence body image perception and attitudes. In the Polce-Lynch, Myers, Kilmartin, Forssmann-Falck, and Kliewer's qualitative study (1998), written narratives were used to probe gender and age patterns of body images, emotional expressions, and self-esteem in adolescents. There were 116 females out of 209 participants from private religious schools in the Southwest. There was a diverse ethnic group. The participants were enrolled in the $5^{\text {th }}$ grade ( 41 females), $8^{\text {th }}$ grade (36), and $12^{\text {th }}$ grade (39). The investigators had hypothesized that media influence would be recounted as a determinate of the girls' body image. Participants were asked an open-ended question about influences on body image. There were only one out of 116 female adolescents who claimed that the media ideal influenced her body image. This result indicated that these participants did not consider media influenced their body image however it was correlated with self-esteem for all age groups. In fact, a major finding for this study was that the girls were both positively and negatively influenced by body image. The authors suggested that media influence was unconscious. 
A meta-analysis on the effect of media on female body image obtained similar results. Holmstrom's (2004) search accrued over 3300 citations. Once the elimination process was completed, 34 studies were left in which effect size estimates were tallied. The results indicated that media had little effect on females' body image, a small effect size $(r=.11)$. Another interesting finding from this meta-analysis was that looking at overweight body images actually increased participants' body satisfaction, but this effect size was moderate $(r=$. 29). The effect size for heavy exposure to the media ideal was negative but of modest size $(r=-.39)$. That is, heavy media consumers also reported greater body satisfaction. These findings from this meta-analysis suggested there were little to no effect of media's influence on female body image.

Magazines. Magazines have been around longer than any other medium. Magazines', or teenzines, exposure gradually increases throughout adolescence. At least half of the female adolescents in America are exposed to teenzines (Currie, 1999; Fine, Mortimer, \& Roberts, 1990; Maine, 2000). Adolescents are enticed by the visual imagery and content. The three major focuses of magazines are appearance, fashion, and dating (Currie, 1999; Maine, 2000; Signorelli, 1997). The message most conveyed throughout female oriented magazines was beautifying the body. Specifically, Guillen and Barr (1994) conducted an analysis of a teenzine, Seventeen (known as the "best friend" of high school girls in America), to characterize nutrition and fitness messages presented between the even years of 1970-1990 (132 teenzines), to evaluate the changes in the messages, and to assess the body shape presented. These 
nutrition and fitness messages were coded and analyzed. The body shape was assessed by measuring bust to waist and hip to waist ratios in photographs of models wearing swimsuits or underwear. As a result, the revealed purpose for following the nutrition and fitness plans was to lose weight and become more attractive. Models' body shapes were found to be less curvaceous while the hip to waist ratio decreased overtime. Further, the nutrition and fitness messages emphasized body image and appearance. Seventeen conveyed the message that the sociocultural ideal body image was an expectation for adolescents. Essentially, magazines subscribed to the fixation of the sociocultural ideal body image.

Research has shown an association between reading teen or fashion magazines and body image attitudes. Field, Cheung, Wolf, Herzog, Gortmaker, and Colditz (1999) conducted a study on magazines influence and weight concerns among females. There were 548 girls in the $5^{\text {th }}$ through $12^{\text {th }}$ grades from the northeastern region of the United States surveyed. The majority of the girls were unhappy with their body image, which was strongly related to their frequency of reading magazines. Pictures from the magazines made a strong impression on the girls' body image. Sixty-nine percent of the girls indicated that magazine pictures influenced their ideal body image and $47 \%$ wanted to lose weight due to this influence. Girls who were frequent readers of magazines were twice as likely to diet and exercise than infrequent readers.

In the Levine, Smolak, and Hayden's (1994) study with 385 girls between 10 to 14 years of age, the girls were surveyed about eating behavior, body 
satisfaction, concerns with being slender, and cues from sociocultural factors. Of this focus group, approximately $60 \%$ of the girls had read at least one of the magazines presented in the study regularly and considered them an important source of information about the sociocultural ideal body image, diet, fitness, and beauty. The one sociocultural factor that emerged as a powerful predictor of investment in thinness, weight management behavior, and disturbed eating was magazines. The results indicated that internalizing the sociocultural ideal body image influenced body dissatisfaction and weight concerns for a significant number of girls.

In an experimental study with college women, Turner, Hamilton, Jacobs, Angood, and Dwyer (1997) assigned young women to two separate waiting rooms with different sets of magazines before they answered a survey about body image and dieting. The young women were exposed either to four fashion magazines or four newsmagazines. The group who were exposed to the fashion magazine recounted more dissatisfaction with their body image, more guilt about eating, and greater fear of getting fat than the group exposed to newsmagazine. This inquiry found that exposure to the media ideal triggered an increase in females' negative feelings about their body image. The print media endorse the thin ideal and present ways to achieve it.

Television. Young people spend more time watching television than any other leisure time activity except sleeping (Strasburger, 1995). In fact, television viewing accounts for almost half of the adolescents' free time (Van Evra, 1998). By the time adolescents' graduate from high school, they will have watched 
around 18,000 hours of television versus spending 12,000 hours in the classroom (Strasburger, 1995). On average, adolescents watch three hours of television per day (Singer \& Singer, 2001; Strasburger \& Wilson, 2002). Clearly, adolescents are being exposed to an inconceivable amount of visual icons conveying media's message.

Television advocates the thin ideal body image. Female characters on television typically have thin and almost perfect bodies, in which their weight is judged as average or below average, and very few overweight models. This was reported by Signorelli's study (1997), in which a content analysis on the reflections of girls in media was conducted. The study examined the body weight of characters and models from 25 favorite adolescents' television programs along with the accompanying commercials. Forty-six percent of the women were seen as thin on television and over half were seen as average weight. In commercials, $55 \%$ of the models were reported as average weight and $32 \%$ as thin.

Regarding the 400 girls between the ages of $10-17$ years surveyed in this study, $61 \%$ of the adolescent girls reported that the female characters in television shows were generally thinner than any women they have known. Essentially, the thin body image was represented in these television shows and commercials. In a similar study, Silverstein and colleagues (1986) explored the role of media in promoting the thin standard. They selected 33 of the most often watched television programs obtained from the Nielsen National Index. Eightytwo female characters over 18 years of age were rated. The raters determined that $69.1 \%$ of the female characters were rated as thin. Moreover, female 
characters were slimmer now compared to their counterparts since 1950. Ultimately, television promotes the sociocultural ideal body image.

According to Victor Strasburger (1995, p. 7), "television is a powerful medium and adolescents are susceptible to it." Since adolescents are in a malleable phase of life, television should influence adolescents' body image in some way since they spend time watching it. In the case of Myers and Biocca's study (1992), the researchers explored the role of television programming in the social construction of reality. This experimental study specifically addressed how television advertising and programming influenced young women's perception of their body image and mood. The participants were 76 college women between the ages of 18 to 24 years from the South. The experiment consisted of watching television programs, filling in a mood-gauging checklist, estimating with a body image detection device, and answering a questionnaire. The participants were divided into four experimental groups and one control group. The researchers indicated that women overestimated their body image. Another result was that women's body image was elastic. According to this study, the participants who watched less than 30 minutes of television and advertising changed the perception of their body image. Overall, these findings suggested that television's message of the ideal body image immediately led to participants' thoughts about their body image which, in turn, led to fluctuations and disturbances of their own body image. Basically, women were influenced by the television messages to be thin and it affected their body image attitude negatively, however, it was only a short-term effect. 
In another experimental study, Cattarin and colleagues (Cattarin, Thompson, Thomas, \& Williams, 2000) examined the role of appearance comparison as a factor in the negative assessment associated with exposure to televised images of attraction. The participants were 180 female college students with an average age of 23 years. The experiment entailed watching 12minute videotapes of commercials, completing a visual analogue scale, and answering questionnaires. Three instructional conditions were created for watching the videotapes: comparison, distraction, and neutral. The comparison condition was to compare themselves with the models; the distraction condition was to focus on the product; and the neutral condition was to watch the commercial like one did at home. In addition, there were two variations of videotapes: The experimental one presented the thin model and the control one presented non-thin models. The participants were assigned one of six different treatment conditions. The results from this experiment concluded that female television icons have the ability to affect, positively or negatively, both mood and body image satisfaction within a normative female group. The participants reported watching around ten hours of television per week, so were exposed more often to television icons. This experiment revealed that the participants in the social comparison condition were more negatively affected than the distraction and neutral conditions. Young women were influenced, positively or negatively, by media's ideal body image.

Correlational studies have provided evidence of a link between media ideal body image and female's body dissatisfaction. Specifically, Tiggemann and 
Pickering (1996) investigated the role of television in adolescents' body dissatisfaction and drive for thinness. The participants were 94 Australian female adolescents with an average age of 15.5 years. They recounted what television programs and how often they watched in a week's time. This study determined the television programs that the girls watched were important. In particular, these programs with the stereotypical women - thin - were positively correlated with body dissatisfaction. Regarding personal assessments, the girls perceived that they were slightly overweight, dissatisfied with their body image, and had a high drive for thinness. This study concluded that specific television programs transmitting the thin ideal body image negatively influenced the adolescents' body image.

In another survey study, Botta (2000) assessed the comparison between Black and White adolescents' perceived media ideal and current body images. A shift has been occurring with non-white ethnic groups being influenced by the media ideal body image especially with non-white thin celebrities' representatives. This study demonstrated that shift. The author tested the comparison of Black and White adolescents' body image with 145 White and 33 Black females with an average age of 15 years. The Black girls were more satisfied with their body image than White girls. They also had larger individual ideal body images than White girls. Botta (2000) found that Black girls compared themselves to media images while viewing television. The Black and White adolescent girls who were heavy viewers idealized television images to the same extent. Furthermore, the more they internalized the media ideal then the 
stronger their drive to be thin, the more dissatisfied they were with their bodies, and the thinner their personal ideal body image. Botta (2000) surmised that there still was a difference in Black girls' responses and the impacts of the media ideal; however, the gap was narrowing.

Music Videos. Music videos are more than television and music; they are self-reinforcing (Strasburger \& Wilson, 2002). When a person hears the song after seeing a particular music video, then that visual imagery of the video pops into her mind. Music videos have altered television viewing, music listening, and record buying habits of adolescents whom defines its audience (Strasburger \& Wilson, 2002). Presently, the average adolescent watches between 30 to 60 minutes per day of music videos (Strasburger \& Wilson, 2002). They are immensely popular with adolescents.

Females on music videos are depicted as sex objects, who are physically attractive, scantily dressed, and provocative dancers. They also represent the media ideal body image. To illustrate, Signorelli (1997) reported that $43 \%$ of the females in music videos were thin with another $32 \%$ at average weight. Television studies rarely separate music videos from television programs when considering the body weight of characters. In any event, music videos more frequently represent the thin ideal than television programs, probably due to wearing less clothes and gyrating provocatively (Strasburger \& Wilson, 2002).

Music videos are believed to have a greater effect on females' body image as the thin ideal is reinforced not only when watching the music video, but also when hearing the song. Studies have indicated that music videos influenced 
female adolescents' body image. Tiggemann and Pickering (1996) investigated the role of television however separated music videos data and female adolescents' body image. Questionnaires were given to 94 Australian girls. Body dissatisfaction and the drive for thinness were assessed. The girls reported watching on average 1.62 hours of music video programs in a week's time. The results indicated that only music videos predicted the drive for thinness $(r=.25$, $p<.05)$. The researchers suggested that music videos' models could be deliberately demonstrating to young women how their bodies should appear and how to behave. The findings also indicated that music videos influenced female adolescents drive for thinness.

In another correlational study, Borzekowski, Robinson, and Killen (2000) examined the relationship between electronic media use and female adolescents' body image. They surveyed approximately 837 ninth graders in California. The ethnic composition was $36 \%$ Latino, $24 \%$ White, $22 \%$ Asian, $8 \%$ Black, and $10 \%$ other. The study found that total media usage was not significantly linked to body image. Only hours spent watching music videos were influential and that relationship was slight. Yet, this relationship disappeared when controlling for level of body fatness and ethnicity. Music videos were the only electronic media in this study to demonstrate a relationship with adolescents' body image (Borzekowski et al., 2000).

In an experimental study, Tiggemann and Slater (2004) tested the effect of the thin ideal as presented in music videos on young women's body image. There were 84 college women between 18 and 30 years old with the average 
age of 20 from Australia participating in this experiment. The experiment entailed watching one of two videotapes, filling in visual analogue scales, and answering questionnaires. There were two videotapes in which one was an appearance tape that had the thin ideal represented and the other a tape that had ordinary looking people and scenes represented. This study acknowledged that the brief exposure to music videos with thin models led to increased body dissatisfaction, but no impact on mood. Tiggemann and Slater (2004) suggested thin models in music videos that influenced the negative body image of adolescents were mediated by social comparison.

To summarize, media are a constant in adolescents' lives. Media reflect the sociocultural ideal body image as well as maintain it. Through modeling, media repeatedly transmit the thin message. Television, magazines, and music videos, the three popular mediums of female adolescents, send a strong vicarious message that adolescents receive and internalize. Research has been presented of different empirical proof, descriptive, correlational, experimental, and personal accounts studies, converging to offer evidence of a link between media influence and female adolescents' body image dissatisfaction. Several inquiries revealed that media influence female adolescents' body image dissatisfaction (Durkin \& Paxton, 2002; Garner, 1997; Levine et al., 1994; Turner et al., 1997). On the other hand, several studies indicated that the media ideal do not influence female adolescents' body image dissatisfaction (Holmstrom, 2004; Polce-Lynch et al., 1998; Tiggemann et al., 2000; Tiggemann \& Pickering, 1996). 
Modeling, exposure, and social comparison are the underlying processes that mediate the influence of the media ideal on body image as demonstrated in these studies. According to the social comparison studies, female adolescents socially compare themselves to the media ideal and report being dissatisfied with their body image on a consistent basis (Cattarin et al., 2000; Garner, 1997; Jaffee \& Lutter, 1995; Tiggemann et al., 1996). The media exposure studies demonstrate a relationship between media exposure of the thin ideal and body image dissatisfaction with heavy viewers (Borzekowski et al., 2000; Field et al., 1999; Levine et al., 1994; Tiggemann \& Pickering, 1996). Furthermore, a few of the media exposure inquiries are unable to determine whether media exposure is a short term or long term effect on female adolescents' body image dissatisfaction (Durkin \& Paxton, 2002; Myers \& Biocca, 1992). Meanwhile in several media studies, media's message is conveyed through modeling of the thin ideal. Through modeling, the influence of the media ideal is related to female adolescents' body image dissatisfaction (Garner, 1997; Tiggemann et al., 2000)

Many researchers agreed that individual differences play an important role in media influence and body image perceptions (Durkin \& Paxton, 2002; Grogan, 1999; Myers \& Biocca, 1992; Thompson et al., 1999). This may be why the results of the media studies varied on body image dissatisfaction. What is established is that media influence body image perceptions just not necessarily body dissatisfaction. 
Overall, these vicarious experiences are shown to be influential on female adolescents' body image. Signorelli (1997) aptly expressed it, "The message sent in even one television show, or movie, or magazine, or advertisement, or music video do not stand alone. They become part of a larger sphere of influence in girls' lives and thus have the power to reinforce - or the potential to challenge - all other messages girls are sent."

\section{Interactions of Direct and Vicarious Experiences}

Direct and vicarious experiences have been evaluated separately; nevertheless, it is informative to consider the interactions of these experiences. The sociocultural agents of family, peers, and media elicited influential as well as noninfluential results on female adolescents' body image when studied separately. Because body image is a lifelong development, any sociocultural agents that interact in female adolescents' lives should influence them in some way.

The sociocultural ideal body image has been established with both direct and vicarious experiences. The thin icon may vary among the sociocultural agents due to years of adaptation. Nevertheless, thin is thin; the difference is a matter of degree. Sociocultural agents endorse a thin ideal. The transmissions of the thin ideal message vary - media are subtle, while the others are direct. One is nonverbally communicated, while the other is verbally communicated. The culmination of these primary sociocultural agents should have a strong impact on female adolescents' body image. 
Levine et al. (1994) surveyed 385 girls between the ages of 10 to 14 years about the influence of family, peers, and media on body dissatisfaction. The majority of girls reported receiving clear messages from media, peers, and family that slenderness was important and attainable through dieting and other methods. In fact, $12.5 \%$ of the girls reported reading at least one magazine regularly and receiving a strong drive for thinness message from peers and parents. The intercorrelations among the sociocultural influences were weak to moderate. The researchers suggested it reflected large individual differences in the amount and source of sociocultural pressure for thinness. The study claimed that body dissatisfaction and weight concerns reflected confirmation of the sociocultural ideal body image.

In another multifactorial study, Dunkley and colleagues (2001) examined the role of sociocultural agents in determining body satisfaction and dietary restraint of adolescent girls. There were 577 Australian girls approximately 15 years of age. Participants answered questionnaires and had their height and weight measured. Many of the girls stated that family, peers, and media influenced their perception of what their body should look like and how they attempted to lose weight. The best predictor of body dissatisfaction was the girl having a larger body size than her peers. The combined influence of these sociocultural agents predicted body dissatisfaction and dietary restraint better than any one sociocultural agent did separately.

McCabe and Ricciardelli (2003) investigated the role of parents, peers, and media in body image and body changes strategies among female 
adolescents. The participants were Australian girls with an average age of 13 years. The researchers determined that parents were a more important communicator of the thin ideal than peers and media. Sociocultural influences from the girls' best friend and mother were important predictors of body changes strategies. Media were not a major predictor of body image and body change strategies, however they encouraged girls to lose weight.

Van den Berg and associates (Van den Berg, Thompson, ObremskiBrandon, \& Coovert, 2002) examined the Tripartite Influence Model with 150 young women between the ages of 18 to 22 years from a southern university. The model proposed that family, peers, and media contributed to the development of body image perceptions and eating disturbances in females. Adjustments were made to the model during the study. Once adjusted, findings of the revised model determined that social comparison mediated the influence of family and media on body dissatisfaction. This, then, influenced dieting and bulimic behaviors. This effect was found especially true for media as they had a strong path leading to appearance comparison. The influences of peers had a direct impact on dieting. This study found that through social comparison the sociocultural agents influenced female adolescents' body image.

Qualitative studies provided another form of evaluation in determining the sociocultural influences on female adolescents' body image. One qualitative study explored sociocultural pressures to be thin. Wertheim and colleagues (1997) queried 30 females around the age of 15 years from Australia. Semistructured interviews explored the influence of family, peers, and media on 
adolescents' body concerns, dieting, and weight watching. One of the strongest correlations in this study was that social comparison led to body concerns. Findings determined a strong role of sociocultural influences leading to healthy and unhealthy body attitudes and eating behaviors. Girls reported that media put forth the strongest pressure to be thin for adolescents. Yet, family and peers reinforced this influence. These findings indicated that sociocultural agents influenced female adolescents' body image.

In another qualitative study, Tiggemann and associates (2000) investigated body concerns of adolescent girls through group interviews. There were 67 females around 16 years of age from Australia. The sociocultural agents in this study were peers and media. The authors found that media and peers, especially media, exerted the most pressure to be thin. The girls wanted to be thinner, however they were not necessarily dissatisfied with their bodies. Results indicated that peers and media influenced adolescent girls, and the girls wanted to be thinner due to this influence.

To sum up, these multifactorial studies confirmed a moderate relationship with sociocultural factors and adolescents' body image dissatisfaction. Three out of the six studies actually conducted intercorrelations analyses among the sociocultural agents and determined that the combination of the agents influenced body dissatisfaction (Dunkley et al., 2001; van den Berg et al., 2002; Wertheim et al., 1997). These investigators suggested that the combination of sociocultural influences was better than any one sociocultural agent separately (Dunkley et al., 2001; van den Berg et al., 2002; Wertheim et al., 1997). Half of 
these multifactorial studies indicated that media had a stronger influence than family and peers (Tiggemann et al., 2000; van den Berg et al., 2002; Wertheim et al., 1997). Visual imagery may have been a factor in this result. Additional research in the interactions of multiple sociocultural agents and their impact on body image would be beneficial in solidifying these outcomes.

\section{Summary and Predictions}

Family, peers, and media have established that the sociocultural ideal body image was thin. They have communicated this thin standard to female adolescents. In particular, media have been a powerful and consistent conveyor of this message (Singer \& Singer, 2001; Strasburger \& Wilson, 2002). Literature has indicated that family, peers, and media as well as their interactions have contributed to the influence of female adolescents' body image. Through direct experiences, family and peers' criticism and teasing have been established as the major contributing traits in influencing female adolescents' body image dissatisfaction. These experiences were direct and immediate. With vicarious experiences, the processes of modeling, exposure, and social comparisons have been substantiated in mediating the influence of the media ideal on female adolescent's body image dissatisfaction. These experiences were indirect, subtle, and cumulative. The interactions of family, peers, and media have been established as affecting female adolescents' body image dissatisfaction; specifically, media has been shown to have a stronger influence than family and peers. In essence, the literature has revealed that the influence of media on female adolescents' body image perceptions has been more consistent than the 
influence of family and peers. This study explores media's influence on body image perceptions relative to other sociocultural factors.

Studies have established that the ideal body images projected thinness. Most researchers have agreed that the media ideal reflected the sociocultural standard (Grogan, 1999; Sault, 1994; Thompson et al., 1999). It, however, has been unclear whether the personal ideal body image also referred to as the ideal body image mirrored the media ideal body image. Studies have used the personal ideal body image and media ideal body image interchangeably as if they were the same degree of thinness. Basically, there has been no to little distinction made between these two ideal body images. Hence, this study examines the relationship between media ideal body image and personal ideal body image.

Several studies have shown that female adolescents have a heavier current body image than the media ideal (Garner, 1997; Thompson et al., 1999; Tiggemann et al., 2000). It has been established that a gap exists between media ideal body image and female adolescents' current body image (Grogan, 1999; Sault, 1994; Tiggemann et al., 2000). The literature has indicated that this discrepancy was a measure of dissatisfaction with adolescents' current body image, whereas when there was no difference, adolescents were satisfied with their body image (Durkin \& Paxton, 2002; Posavac et al., 1998; Turner et al., 1997). Several studies also have ascertained that young women were not necessarily dissatisfied with their body image even when a difference existed (Botta, 2000; Myers \& Biocca, 1992; Tiggemann et al., 2000). Thus, this study 
investigates the relationship between media ideal body image and current body image.

The ultimate goal of this study is to elaborate and advance the findings from prior research on the effect of media on female adolescents' perceptions of their satisfaction with their body image. Therefore, the following predictions were posed:

1. Female adolescents perceive the media ideal body image as thin.

2. Female adolescents perceive their personal ideal body image as thin.

3. Female adolescents' personal ideal body image is correlated with the media ideal body image.

4. Female adolescents are dissatisfied with their current body image.

5. Among female adolescents, the discrepancy between personal ideal body image and current body image is inversely correlated with their satisfaction of their body image.

6. Female adolescents perceive that the media ideal body image is thinner than their current body image.

7. Among female adolescents, the discrepancy between media ideal body image and current body image is inversely correlated with their satisfaction of their body image.

8. When asked to rank the relative influence of several sociocultural agents, female adolescents will rate media as the strongest. 
9. Female adolescents who rank media as a more important influence on their ideas about their bodies are less satisfied with their body image than those who rank media as less important. 


\section{CHAPTER 3}

\section{METHOD}

The previous chapter presented nine predictions about female adolescents' perceptions of their body image and the role of media. In order to test these predictions, data were collected from the typical college female regarding perceived body image, personal ideal body image, media ideal body image, and media influence. This study was designed to assess the interactions of perceived body image with media ideal body image and personal ideal body image. It will also examine the relationship between media ideal body image and personal ideal body image. The influences of sociocultural factors on female adolescents' perceptions of their body image will be investigated as well. This chapter explains the measures, participants, research settings, and procedures that were employed to test the predictions.

\section{Measures}

\section{Body Mass Index}

Developed by the National Center for Health Statistics (Centers for Disease Control and Prevention, 2004; Jaffe \& Lutter, 1995), the Body Mass Index (weight/height ${ }^{2}$ ) serves as a measure of weight for height. This index was selected to gage the reality of the participants' perceptions of their body image. Self-reported height and weight of the participants were calculated to identify 
their Body Mass Index (BMI). Studies have found self-reported height and weight were valid (Bulik, Wade, Heath, Martin, Stunkard, and Eaves, 2001; Field et al., 1999; Rosenblum \& Lewis, 1999). This index established whether people were underweight, normal, overweight, or obese according to the BMI Table of the Centers for Disease Control and Prevention (CDC; 2004). The BMI norms are as follows: underweight range is $<19$, normal range is 19 up to 25 , overweight range is 25 up to 30 , and obese is 30 and over.

\section{Figure Rating Scale}

Stunkard, Sorenson, and Schulsinger (1983) developed the Figure Rating Scale in the early 1980 s in order to measure the perception of body image. It has been used in numerous body image studies ever since (Monteath \& McCabe, 1997; Must, Phillips, Stunkard, \& Naumova, 2002; Thompson \& Altabe, 1991). This scale was selected because of its simplicity, easy administration, and robustness.

Figure 1

Figure Rating Scale

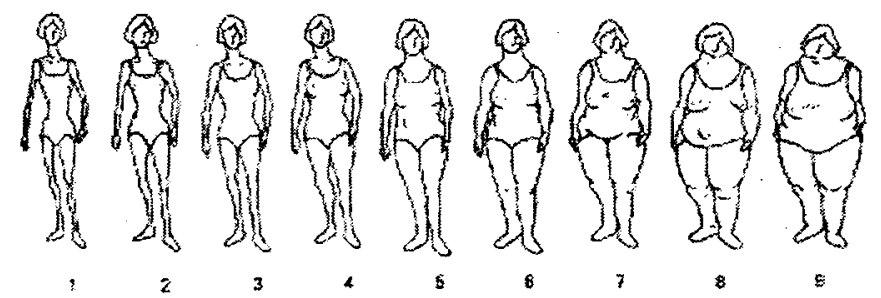


The figure drawing scale consisted of nine female silhouettes that ranged from extremely thin to extremely obese. Numbers 1 through 9 were assigned to rate the figures with " 1 " designating the extremely thin figure and " 9 " the extremely obese figure. Participants were given 3 figure rating scales to identify a figure that represented their (a) current body image, (b) ideal body image, and (c) media ideal body image (see Appendix A). These ratings were calculated to assess the discrepancies as well as the relationships between (a) ideal body image and media ideal body image, (b) current body image and ideal body image, and (c) current body image and media ideal body image. The discrepancies (a) between current body image and the ideal body image and (b) between current body image and media ideal body image constituted measures of body dissatisfaction. The Figure Rating Scale had a good test-retest reliability (.89) and adequate validity according to Thompson and Altabe (1991).

Bulik, Wade, Heath, Martin, Stunkard, and Eaves' study (2001) established norms for the Figure Rating Scale using a large Caucasian sample. Data were collected from sets of twins both females and males ranging in age from 18 to 100 . The data from 16,728 female twins established (a) norms for females on the Figure Rating Scale, (b) BMI norms for females on the Figure Rating Scale, and (c) obesity and thinness classifications. The classification of thinness on the Figure Rating Scale provides an external standard to document thinness. Bulik and associates (2001, p. 1523) indicated that the Figure Rating Scale was a "potentially accurate method" to classify individuals as thin. Figures 1 through 4 of the Figure Rating Scale represented thinness with figure 4 as the 
"optimal cut-off." For the present study, the classification of thinness will determine whether the ideal body images as rated on the Figure Rating Scale by the participants are thin.

Sociocultural Attitudes Towards Appearance Questionnaire-3

Kevin Thompson developed the Sociocultural Attitudes Towards Appearance Questionnaire to measure media influence (see Appendix B). The third version of the Sociocultural Attitudes Towards Appearance Questionnaire-3 (SATAQ-3) was validated in 2004 (Thompson, van den Berg, Roehrig, Guarda, \& Heinberg, 2004).

The 30-item SATAQ-3 was comprised of four subscales assessing media influence in the following areas: information, 9 items; pressure, 7 items; general internalization, 9 items; and athlete internalization, 5 items. Each item was rated on a 5-point scale ranging from 1 - "definitely disagree" to 5 - "definitely agree." The information subscale evaluated media as an information source of the thin standard (e.g., TV programs are an important source of information about fashion and "being attractive."). General internalization assessed internalizing the thin ideal represented in media (e.g., I compare my body to the bodies of people who are on TV.). Athlete internalization assessed internalizing the athletic body represented in media (e.g., I compare my body to that of people who are athletic.). The pressure subscale evaluated the pressure felt from media to meet the thin standard (e.g., l've felt pressure from TV or magazines to lose weight.). The total scale analyzed the overall media influence (Thompson et al., 2004). 
Eight items $(3,6,9,12,13,19,27$, and 28$)$ were reversed keyed, so they were recoded for analyses.

The SATAQ-3 had a high convergent validity with measures of body image and eating disturbance (Thompson et al., 2004). The SATAQ-R, a second version of the SATAQ, had an internal consistency of .86 and test-retest validity of .86 (Thompson \& van den Berg, 2002).

\section{Body Image/Media Survey}

The Body Image/Media Survey was developed to obtain additional information about media and body image. This survey measured body image satisfaction, ranking of sociocultural factors, and media influence (see Appendix C). Survey experts and young women reviewed this instrument to (a) provide feedback, (b) check for clarity, and (c) make suggestions for improvement.

Nine scale items were rated on a 5-point scale ranging from 1 - "strongly disagree" to 5 - "strongly agree." The body image satisfaction scale consisted of 6-items assessing body image satisfaction (e.g., I am satisfied with my body shape.). This scale queried body shape, weight, and body aspects. Three items $(2,4,6)$ were recoded on the body image satisfaction scale, as they were reverse ordered. The 3 -item media ideal influence scale evaluated media influence on body image (e.g., The way women appear in music videos influences how I would like to look.).

Sociocultural factors were ranked according to how important they were in influencing young women's ideas about their body image. The five factors were 
family/guardians, peers, self, media, and males. A 1 was assigned to the "most important" influence and a 5 for the "least important."

Two personal history questions were asked about cosmetic surgery and eating disorders, because they have been found to impact perceptions of body image (Grogan, 1999; Jackson, 1992; Thompson et al., 1999). With regards to media exposure, the participants were asked to write-in how many hours per week they were engaged in media. The last section presented two open-ended questions about media influence and its message.

\section{Demographic Indices}

Participants also provided demographic information about their age, ethnicities, and permanent geographic locations (see Appendix D).

\section{Participants}

Participants were recruited from the Piedmont area of Virginia during the summer and fall of 2005 . Fifteen colleges were identified in the region that served primarily young women between 18 to 21 years of age. An administrator was contacted at each college about initial interest in the study. A packet of information about the study was sent to the identified colleges. The packet contained the following materials: (a) introductory letter with the details of the study (see Appendix E), (b) Informed Consent Agreement for the participants (see Appendix F), (c) study procedures (see Appendix G), and (d) the survey packet. A follow-up telephone call was made to determine their interest and availability. 
Four sites were selected based on three types of higher education systems: two-year, four-year, and career. Directors from each location granted permission to conduct the study at his/her site. Dates, times, and study setup were arranged at a meeting with the administrator. The Institutional Review Board's letter of approval for this study was given to each participating college. A private women's college was selected for the four-year site. At this residential college, there were around 800 students (J. Vondrasek, personal communication, September 16,2005$)$. The other colleges were commuter schools. The two-year site was a community college with over four thousand students (T. Atkins-Brady, personal communication, June 30, 2005). Based on the limited enrollment of young women between 18 to 21 years of age, two branches of the career college participated in this study. Less than two hundred students were enrolled at the first career college site (S. Gough, personal communication, July 19, 2005), while over three hundred students were enrolled at the second career college site (S. Coleman, personal communication, September 30, 2005).

Participants consisted of 108 young women from two-year, four-year, and career colleges in the Piedmont area of Virginia. They ranged in age of 18 to 21 years with an average age of $19.63(S D=1.04)$. Fifty-one percent of the females were white, $32 \%$ were black, and the rest belonged to several nonwhite minority groups. 


\section{Research Settings}

Research settings varied among the college sites. The two-year college setting was located outside between two buildings where students generally entered. This study was conducted during five days in July. Eighteen surveys were completed at this time. With a low response rate during the summer, the administrator granted permission for me to return during the fall semester. The return visit occurred during two days in September. Twenty-five surveys were collected. Forty-three surveys were completed at the two-year college. This accounted for $40 \%$ of the total surveys collected.

The second study site was at the career college during mid July. The research setting was situated inside the main entrance of their building where students generally passed. Eight surveys were gathered over the five days. These surveys accounted for $7 \%$ of all the surveys collected.

At the four-year college, the study was conducted during two days in September. This study site was positioned inside the main entrance of the dining hall. The collection of surveys stopped at the four-year college once the same number as gathered at the two-year college was reached. That is, 43 surveys were collected which accounted for $40 \%$ of the overall surveys gathered.

The final study site was at the second branch of the career college. The study was conducted during four days in October. This setup was different in that the Director of Healthcare Education implemented the study. I met with her prior to conducting the study in order to discuss procedural protocol. With permission from the instructors, the director approached all the day classes prior 
to their break time in order to recruit young women for the study. The volunteers met with her during break. She followed the procedures as outlined below. Each day the collected surveys were placed in a folder. At the end of the week, I picked up the completed surveys and study materials. Fourteen surveys were completed, which accounted for $13 \%$ of all the surveys compiled.

\section{Procedures}

A table and chairs were provided at the study sites. The research locations were highly trafficked areas on campus. At each location, a site folder was maintained that consisted of procedures, site information sheet (see Appendix $\mathrm{H}$ ), and interested-in-the-results form (see Appendix I). A "Young Women's Body Image Study" sign was designed with the intent of drawing young women to the table. This sign was placed on top of the table along with the study materials.

At the two-year college where the study was first conducted, the sign and materials read that the survey took $\mathbf{3 0}$ minutes to complete; however, most of the young women completed the survey packet in 15 minutes. Therefore, the sign and scripted instructions were amended to reflect this time change after the first site.

Many young women approached the table asking questions about the body image study. With the young women who agreed to participate, the purpose of the study was explained. Volunteers were informed that anonymity was guaranteed, as the survey packet was to be returned in an envelope without any identifying information. I handed out the Informed Consent Agreement and 
an envelope that contained a list of body image definitions and the survey packet. Participants were asked to review the Informed Consent Agreement before signing it. They were not informed that the body image study was focused on media influence, except if they read the title on the Informed Consent Agreement. Participants were apprised that it would take approximately 15 minutes to fill-in the surveys. They were told that the survey packet contained a cover sheet with demographic information, three Figure Rating Scales, the Sociocultural Attitudes Towards Appearance Questionnaire-3 (SATAQ-3), and the Body Image/Media Survey. Participants were asked to select the best answer that reflected how they thought and felt about body image. They were instructed that when they had questions to ask me. Participants proceeded to fillin the survey packet. Once completed, the packets were returned along with the signed Informed Consent Agreement on top of the envelope. At that time, participants were informed that if they were interested in receiving the results from this study, then they were to write their e-mail address on the "Interested in the Results" form. If they did not have an e-mail address, they were told to write down their last name and address. Once this was completed, participants were thanked for their participation and time with the study. 


\section{CHAPTER 4}

\section{RESULTS}

The present investigation was designed to examine the effect of media on female adolescents' satisfaction with their body image. The groundwork was laid in the last chapter by describing the instruments, participants, and procedures for this study. With that established, the female adolescents' responses to the surveys were analyzed and the results presented in this chapter. Results have been organized by demographic data, descriptive data, and predictions.

\section{Demographic Data}

Table 1 presents the means and standard deviations of age, height, weight, and Body Mass Index. Additional demographic data provided an overall profile of the participants in this study. 
Table 1

Means and Standard Deviations for the Demographic Indices

\begin{tabular}{lccc}
\hline & $N$ & Mean (M) & Standard Deviation \\
& 108 & 19.63 & 1.04 \\
\hline Age & 108 & 64.31 & 2.86 \\
Weight & 108 & 150.75 & 42.63 \\
Body & 108 & 25.52 & 6.53 \\
\hline
\end{tabular}

Participants were between 18-21 years of age with an average age of 19 years 7 months. Age cohorts consisted of twenty-nine 18 -years old $(27 \%)$, fortytwo 19-years old (39\%), twenty-one 20-years old (19\%), and sixteen 21-years old (15\%). Within this group of college women, ethnic factions were composed of $51 \%$ white (55), $32 \%$ black (34), $14 \%$ "other" (15), and $3 \%$ unreported (4).

Geographic locations were requested as some college women may have had a permanent domicile outside the Piedmont area in Virginia. Sixty-eight percent (73) of the participants hailed from the Piedmont area with another $10 \%$ (11) from outside this area but still in Virginia. Overall, $86 \%$ (93) of the respondents were from the Mid Atlantic region while $14 \%$ (15) resided outside this region.

Participant's mean height was 5 feet, 4 inches and ranged from 4' 10 " to 5'11'. Median height for this group was 5'4". A 151 pounds was the mean weight of this group. They weighed between 92 to 295 pounds with a median 
weight of 139 pounds. Respondents' mean weight was higher than their median weight due to the data being positively skewed; that is, a few individuals distorted the distribution upward by being excessively overweight.

Self-reported weight and height were used to calculate Body Mass Index (weight/height ${ }^{2}$ ). The mean BMI for these participants was 25.52. A BMI of 25 up to 30 was considered overweight. Median BMI was 23.72, which was in the normal range. This variation between the mean and median BMI score was due to the top $10 \%$ of the participants having a BMI of 33.4 and more. According to the BMI Table for the Centers for Disease Control and Prevention (2004), 7\% (8) of the respondents were in the undenweight range, $50 \%(54)$ were in the normal range, $23 \%(25)$ were in the overweight range, and $20 \%(21)$ were in the obese range.

\section{Descriptive Data}

\section{Figure Rating Scales}

In Table 2, the means and standard deviations of the Figure Rating Scales are displayed for the current body image, personal ideal body image, and media ideal body image. (See Appendix A for the Figure Rating Scales.) 
Table 2

Means and Standard Deviations for the Figure Rating Scales

\begin{tabular}{lccc}
\hline & $N$ & $M$ & $S D$ \\
\hline Current body image & 107 & 4.29 & 1.20 \\
Ideal body image & 108 & 3.53 & .74 \\
Media ideal body image & 108 & 2.03 & .74 \\
\hline
\end{tabular}

The Figure Rating Scale consisted of 9 figures ranging from 1 - "extremely thin" to 9 - "extremely obese." Figure 4 represented the mean rating of the current body image. Ratings ranged from figure 2 to 8 with a median rating of 4 . One respondent failed to select a figure that reflected her current body image.

The mean rating of the personal ideal body image was midway between figures 3 and 4 . Ratings of the personal ideal ranged from figure 2 to 5 . A median rating for the ideal body image was 3.5 .

With the media ideal body image, figure 2 reflected the mean response of these participants. Media ideal body image ratings ranged from figure 1 to 4 with a median rating of 2 .

Sociocultural Attitudes Towards Appearance Questionnaire-3

Table 3 displays the means and standard deviations of the Sociocultural Attitudes Towards Appearance Questionnaire-3 (SATAQ-3). This media influence measure was comprised of four subscales (information, pressure, general internalization, and athlete internalization) and a total scale. (See Appendix B for the SATAQ-3.) 
Table 3

Means and Standard Deviations for the Sociocultural Attitudes Towards Appearance Questionnaire-3

\begin{tabular}{lccc}
\hline & $N$ & $M$ & $S D$ \\
\hline Media Information & 108 & 26.56 & 8.13 \\
Media Pressure & 108 & 21.99 & 7.53 \\
General Internalization & 108 & 25.75 & 8.35 \\
Athlete Internalization & 108 & 15.36 & 4.60 \\
Total & 108 & 88.88 & 22.55 \\
\hline
\end{tabular}

Each item from the SATAQ-3 responded to using a 5-point scale ranging from 1 - "definitely disagree" to 5 - "definitely agree." Scores for the items on each of the four subscales and total scale were then summed. Media as the information source of the thin ideal was a 9-item subscale that ranged in scores from a minimum of 9 to a maximum of 45 and a midpoint of 27 . Media pressure to meet the thin standard had scores that ranged from a minimum of 7 to a maximum of 35 with 21 the mid range on this 7 -item subscale. The 9-item general internalization of the thin standard subscale spanned from 9 to 45 points (27 mid range), however, the maximum range for this group was 42 . None of the participants definitely agreed with every item on the general internalization subscale. The 5-item subscale of the internalization of the athletic body message ranged from 5 to 25 points with 15 the mid range. The athlete 
internalization subscale had 2 missing values from 2 participants: one was item 20 and the other was item 23 . These missing values were replaced with the average of the participant's athlete internalization subscale scores. With the 30 item SATAQ-3 scale of media influence, the scores had a possible range from a minimum of 30 to a maximum of 150 ; however, this group's maximum range was 141. None of the participants definitely agreed with every media influence item. Ninety was the middle score of the range on the total scale of the SATAQ-3.

\section{Body Image/Media Survey}

Means and standard deviations for the Body Image/Media Survey (BIMS) are exhibited in Table 4. This media and body image measure consisted of body image satisfaction, ranking of sociocultural influences, media ideal influence, media exposure, and media influence. (See Appendix C for the Body Image/Media Survey.) 
Table 4

Means and Standard Deviations for the Body Image/Media Survey

\begin{tabular}{lccc}
\hline & $N$ & $M$ & $S D$ \\
\hline Body Image Satisfaction & 108 & 17.55 & 5.54 \\
Media Ideal Influence & 108 & 7.98 & 3.46 \\
Media Exposure (hrs./wk.) & 106 & 11.09 & 17.44 \\
Sociocultural Influences & & & \\
$\quad$ Family/Guardians & 107 & 3.00 & 1.44 \\
Peers & 107 & 3.01 & 1.01 \\
Self & 107 & 2.23 & 1.42 \\
Media & 107 & 3.57 & 1.45 \\
Males & 107 & 3.20 & 1.14 \\
\hline
\end{tabular}

Responses to each item of the body image satisfaction and media ideal influence scales were on a 5-point scale of 1 - "strongly disagree" to 5 - "strongly agree." Scores for the items in each set were tallied. The 6-item body image satisfaction scale ranged in scores from 6 to 30 with 18 the midpoint. There was a missing value for item 4 so an average of the individual's scores were calculated and then replaced the missing value. A coefficient alpha of .82 revealed that the body image satisfaction scores were reliable for participants in this study. With the 3-item media ideal influence scale, the scores ranged from 3 to 15 with a mid range of 9 . Twenty-one participants strongly disagreed with every item on this scale that media influenced how they would like to look, 
whereas 4 participants strongly agreed with that assessment. A coefficient alpha of .88 indicated that the media ideal influence scores were reliable.

Media exposure was another media variable of the BIMS. Two participants did not respond to the media exposure item. The mean media exposure was 11.09 hours per week with a median time of 7 hours per week. Time spent engaged in media spanned from 0 to 140 hours per week. Two participants spent more than 100 hours per week engaged in media, while the rest of the participants spent 40 hours and less per week. These two extreme cases of media exposure accounted for the higher mean score.

The ranking section in this survey consisted of 5 sociocultural factorsfamily/guardians, peers, self, media, and males-that influenced female adolescent's ideas about their body. Sociocultural factors were ranked from 1 "most important" to 5 - "least important." There was one missing value for all of the sociocultural factors, because one participant did not respond to this section. Twenty-five out of the 108 participants (23\%) gave the same ranking to one or more of the sociocultural factors; therefore, averages were computed from their ranking responses. The range was 1 to 5 with 3 as the midpoint. Median for each factor was as follows: 2 for self; 3 for each of the family/guardians, peers, and males; and 4 for media.

The personal history items revealed no reported cases of cosmetic surgery and 12 reported cases of eating disorders. Of the 12 cases of eating disorders, there were 4 participants with anorexia nervosa, 6 with bulimia, and 2 did not report the type of eating disorder. When asked the length of time of their 
eating disorder, 4 cases reported a year or less, 2 cases reported two years, 1 case reported three years, 1 case reported four years, 2 cases reported five years, and 2 cases did not respond. In the written response section regarding the type and length of time with an eating disorder, half (6) of the female adolescents stated that they no longer have an eating disorder, while the other half did not designate.

In the last section of the BIMS, there were two open-ended questions regarding media influence. All data from the responses were coded then pared down to the categories posted in Tables 5 and 6.

Table 5 displays the responses regarding media influence on female adolescent's feelings about their bodies. 
Table 5

How does the way females appear in media influence how you feel about your body?

Media influence in how you feel about your body Frequencies $\%$

It doesn't 28 25.9

Be thinner 12

Bad 12

Change how I look 11 10.2

Self-conscious 10

9.3

Good

8

7.4

Fat 7

6.5

Want a healthy body 5

\section{6}

Learn about style from media models 5

4.6

Compare myself to how they look

4

Want to look like them

4

3.7

No response

2

Note. $N=108$.

Twenty-six percent of the participants indicated that the models represented in media did not influence their feelings about their bodies. Seventyfive percent (81) of the participants responded to one of the top 6 categories about media influence of their body image. 
With regards to the second open-ended question, the female adolescent were asked about the most powerful message of the female body that media communicated. Table 6 presents their responses.

Table 6

What is the most powerful message about the female body that media convey to you?

Media's most powerful message about the female body Frequencies \%

Be thin

58

53.7

Appearance is important

12

11.1

Have a fit and healthy body

10

9.3

Lose weight

6

5.6

Nothing

6

5.6

Be sexy

4

Have a shapely body

4

3.7

Be content with your body

4

3.7

No response

4

3.7

Note. $N=108$.

Fifty-four percent of the respondents felt that the most powerful message of media was to be thin. The other categories had a response rate of $11 \%$ and under. The top three responses garnered $74 \%(80)$ of the participants' views of media's most powerful message about the female body. 


\section{Predictions}

\section{Prediction 1}

To test the first prediction that female adolescents perceive the media ideal body image as thin, a comparison of the female adolescents' ratings of their media ideal body image was made with Bulik, Wade, Heath, Martin, Stunkard, and Eaves' thinness classification (2001). In general, Bulik and associates (2001, p. 1523) recommended that the Figure Rating Scale was a "potentially accurate method" to classify thinness. Figures 1 through 4 of the Figure Rating Scale represented thinness with figure 4 as the "optimal cut-off."

All of the respondents $(N=108)$ selected ratings 1 through 4 . The mean rating of the media ideal body image was $2.03(S D=.74)$. Figure 2 of the Figure Rating Scale reflected the average media ideal body image. This met the authors' thinness classification.

Fifty-four percent (58) of the participants expressed that the thin ideal body image was the most powerful message media conveyed to them. As one young woman wrote, "Be thin! It is conveyed as the only healthy and pretty body." This "be thin" message echoed the participants' media ideal rating that the media ideal was thin.

\section{Prediction 2}

To examine the second prediction that female adolescents perceive their personal ideal body image as thin, the Figure Rating Scale was employed to assess the participants' perceptions of their ideal body image. The thinness classification recommended by Bulik and colleagues (2001) was used once again 
to gage whether the personal ideal body image was thin. Figures 1 through 4 on the Figure Rating Scale represented thinness.

Ninety-one percent (98) of the participants $(N=108)$ selected figures 2 through 4 with no one selecting figure 1 . The mean rating of the personal ideal body image was $3.53(S D=.74)$, falling midway between figure figures 3 and 4 . Female adolescents' personal ideal body image was determined thin by Bulik and associates' thinness classification (2001).

\section{Prediction 3}

To test the third prediction that female adolescents' personal ideal body image is correlated with the media ideal body image, a Pearson product-moment correlation was computed to determine whether there was a relationship between female adolescents' personal ideal body image and media ideal body image of the Figure Rating Scales. A positive linear correlation was found between personal ideal body image and media ideal body image $(r=.27, p<.01)$. The effect size was medium as set forth by Cohen's "medium" effect size conventions for correlations (i.e., . 3; 1988, chap. 3). When the personal ideal increased, the media ideal also increased.

Frequencies of the discrepancy scores between media ideal and personal ideal indicated that $90 \%(97)$ of the respondents reported the media ideal as thinner than their personal ideal. A dependent $t$-test was conducted to determine whether the media ideal was thinner than the personal ideal body image.

Results indicated that the media ideal body image $(M=2.03, S D=.74)$ was thinner than the personal ideal body image $(M=3.53, S D=.74), t(107)=17.42$, 
$p<.001$. The media ideal was considerably thinner than the personal ideal with a mean difference of $1.51(S D=.90)$. The effect size $\left(E S=M_{P}-M_{M} / S D_{1}\right)$ was calculated $(d=1.68)$. It was twice as big as Cohen's "large" effect size (i.e., .8; 1988, chap. 5).

Pearson correlations were also computed to ascertain whether there were any linear relationships among the ideal body images and body image and media influence measures. The discrepancy between personal ideal body image and media ideal body image was positively correlated with $\mathrm{BMI}(r=.42, p<.001)$, indicating that participants with high BMls generally had a larger discrepancy between their personal ideal and the media ideal. This effect size was medium (Cohen, 1988). Other than being linearly correlated with the personal ideal, the media ideal did not have a significant linear relationship with any of the body image and media influence measures.

Table 7 displays the correlations of the personal ideal with other body image and media influence measures. 
Table 7

Correlations of Personal Ideal Body Image and Current Body Image with Body Image Satisfaction, Media Ideal Influence, SATAQ-3 subscales, Total SATAQ-3, and $\mathrm{BMI}$

\begin{tabular}{lcc}
\hline & Personal Ideal Body & Current Body \\
& Image & Image \\
& $(N=108)$ & $(n=107)$ \\
\hline Body Image Satisfaction (BIMS) & -.14 & $-.60^{\star \star}$ \\
Media Ideal Influence (BIMS) & -.12 & .08 \\
Media Information (SATAQ-3) & $-.20^{\star}$ & -.06 \\
Media Pressure (SATAQ-3) & .02 & $.31^{\star \star}$ \\
General Internalization (SATAQ-3) & $-.19^{\star}$ & .12 \\
Athlete Internalization (SATAQ-3) & $-.24^{\star}$ & .05 \\
Total SATAQ-3 & -.18 & .13 \\
BMI & $.55^{\star \star}$ & $.82^{\star \star}$ \\
\hline
\end{tabular}

Note. ${ }^{\star} p<.05 .{ }^{\star \star} p<.001$.

Personal ideal body image was negatively correlated with three SATAQ-3

subscales: media information $(r=-.20)$, general internalization $(r=-.19)$, and athlete internalization $(r=-.24)$. These were small effect sizes as stipulated by Cohen's "small" effect size for correlations (i.e., .1; 1988, chap. 3). The thinner the participants' personal ideal body image, the more they agreed with the media message about thinness, the more they agreed that they had internalized that 
message, and the more they agreed they had internalized the notion that they should have an athletic physique. The personal ideal was also significantly correlated with the body mass index $(r=.55)$. This effect size was large as set forth by Cohen's "large" effect size for correlations (i.e., .5, 1988, chap. 3). Heavier female adolescents tended to select a heavier personal ideal.

\section{Prediction 4}

Two measures were implemented to determine whether female adolescents were dissatisfied with their current body image. They were (a) the discrepancy scores between current body image and ideal body image of the Figure Rating Scales, and (b) the body image satisfaction scale of the Body Image/Media Survey.

A dependent $t$-test was administered to compare the ratings of current body image with personal ideal body image. Ratings from 107 participants were evaluated because one individual did not report her current body image. First, the discrepancies from the current body image and the ideal body image were calculated. The dependent $t$-test evaluated body image satisfaction/dissatisfaction. A discrepancy constituted a measure of body dissatisfaction.

Frequencies of the discrepancy scores revealed that $63 \%(68)$ of the participants had a larger current body image than personal ideal, $30 \%(32)$ had no difference, $6 \%(7)$ had a larger personal ideal than current body image, and $1 \%$ (1) had no score. Results of the dependent $t$-test indicated that the average personal ideal body image $(M=3.54, S D=.75)$ was thinner than the average 
current body image $(M=4.29, S D=1.20), t(106)=8.11, p<.001$. The personal ideal body image was significantly thinner than the current body image, a mean difference of $.75(S D=.96)$. The effect size $(d=.78)$ was large according to Cohen's effect size conventions (1988). The results also indicated that a statistically significant correlation existed between current body image and ideal body image $(r=.60, p<.001)$. The effect size was large (Cohen, 1988). A heavy current body image generally yielded a heavy personal ideal. A positive or negative discrepancy score between the current body image and personal ideal body image signified body image dissatisfaction; that is, two thirds of the participants were dissatisfied with their body image and almost a third of them were satisfied.

Table 7 also presents Pearson correlations that were computed to determine whether there were any linear relationships with the current body image and body image and media influence measures (See page 98.). Current body image was negatively correlated with body image satisfaction $(r=-.60)$. The effect size was large (Cohen, 1988). Current body image was positively correlated with media pressure $(r=.31)$, a medium effect size (Cohen, 1988). The heavier the female adolescent's current body image, the more they felt the pressure from the media to be thin and the less they were satisfied with their body. Current body image also was significantly correlated with the body mass index $(r=.82)$. This effect size was large (Cohen, 1988). That is, thin female adolescents usually selected a thin personal ideal. 
The 6-item body image satisfaction scale was also used to ascertain whether the female adolescents $(N=108)$ were satisfied with their body image. The mean for body image satisfaction was $17.55(S D=5.54)$. The range for this scale was 6 to 30 with a midpoint of 18 . There were $55(51 \%)$ respondents who fell below the midpoint, $7(7 \%)$ respondents at the midpoint, and $46(42 \%)$ respondents who were above the midpoint. Figure 2 presents the distribution of the body image satisfaction scores.

Figure 2

Body Image Satisfaction

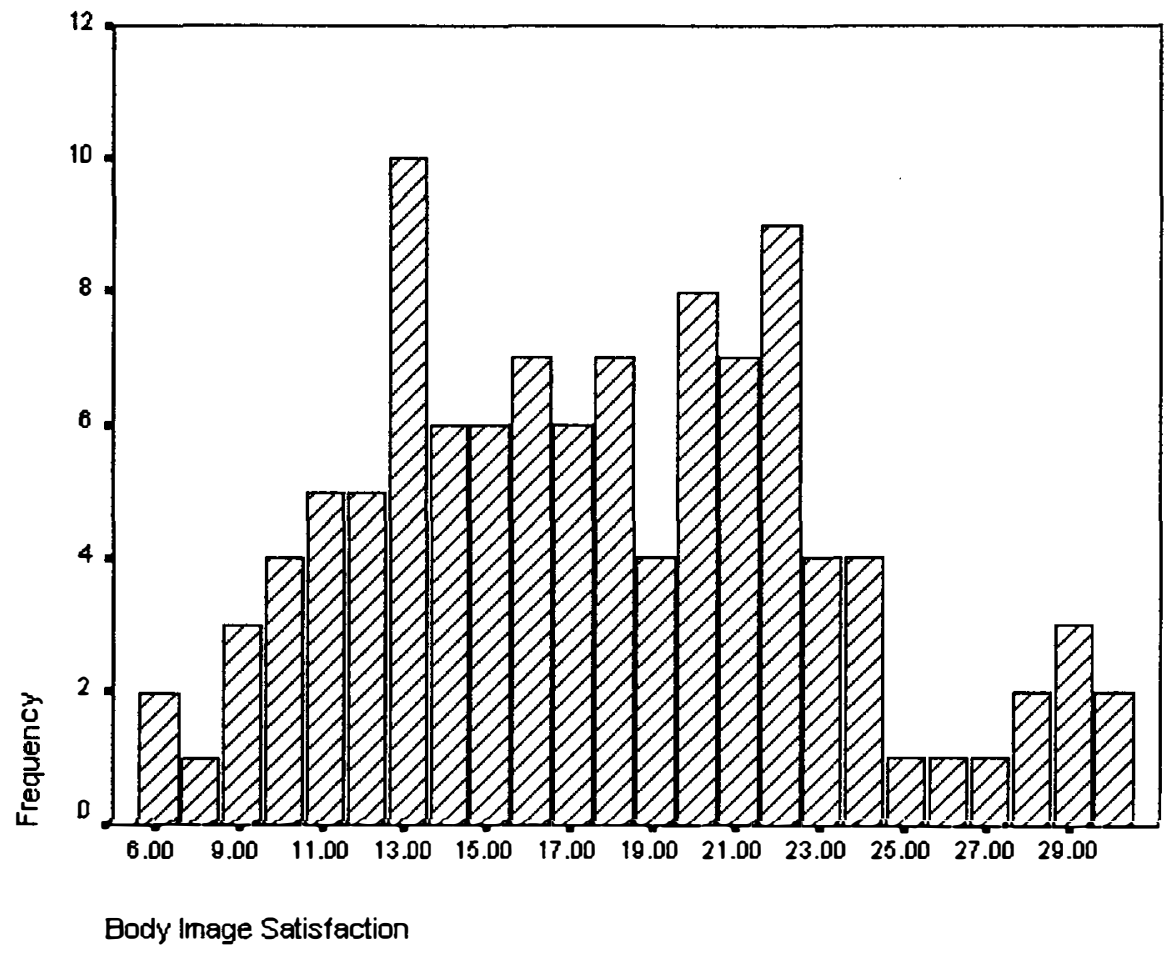

Frequencies of the total scores on the body image satisfaction scale were heavily distributed between two modes, 13 and 22 . Sixty-five percent (70) of the participants' total scores on the body image satisfaction scale were distributed 
from 13 to 22 . Eighteen percent (20) of the participants fell below mode 13 and $17 \%$ (18) were above mode 22 . If the scale was sectioned into thirds according to the respondents' total scores, above 20 was the first third accounting for $32 \%$ (35) of the participants, 15 through 20 was the second third accounting for $35 \%$

(37) of the participants, and 6 through 14 was the last third accounting for $33 \%$ (36) of the participants.

Table 8 presents the items on the body image satisfaction scale along with the participants' responses. 
Table 8

Percentages and Frequencies of the Items on the Body Image Satisfaction Scale

\begin{tabular}{|c|c|c|c|}
\hline & $\begin{array}{c}\text { Strongly } \\
\text { Agree/Agree }\end{array}$ & Neutral & $\begin{array}{c}\text { Strongly } \\
\text { Disagree/Disagree }\end{array}$ \\
\hline \multicolumn{4}{|l|}{ I am satisfied with my body } \\
\hline shape. & $47 \%(51)$ & $21 \%(23)$ & $32 \%(34)$ \\
\hline \multicolumn{4}{|l|}{ I like the appearance of my } \\
\hline stomach and hips. & $26 \%(28)$ & $19 \%(21)$ & $55 \%(59)$ \\
\hline \multicolumn{4}{|l|}{ I am pleased with how I look in } \\
\hline a swimsuit. & $24 \%(26)$ & $26 \%(28)$ & $50 \%(54)$ \\
\hline \multicolumn{4}{|l|}{ I am not or should not be on a } \\
\hline diet. & $38 \%(41)$ & $16 \%(17)$ & $46 \%(50)$ \\
\hline I am satisfied with my weight. & $34 \%(37)$ & $18 \%(19)$ & $48 \%(52)$ \\
\hline \multicolumn{4}{|l|}{ I am pleased with the way } \\
\hline clothes fit me. & $45 \%(49)$ & $29 \%(31)$ & $26 \%(28)$ \\
\hline
\end{tabular}

Note. $N=108$.

Two individuals were satisfied with every item and two individuals were dissatisfied with every item on this scale. Otherwise, results indicated that the participants' satisfaction or dissatisfaction responses varied from item to item; that is, they may have been dissatisfied with one item while satisfied with another. Almost half of the participants were satisfied with their body shape, while a third were dissatisfied and a fifth neither satisfied nor dissatisfied. Some 
of the participants were pleased with the way clothes fit. Almost half of them were dissatisfied with their weight. Half of the female adolescents were not pleased with the way they looked in a swimsuit. Over half of them did not like the appearance of their stomach or hips. Some of the participants were or thought they should be on a diet.

\section{Prediction 5}

The fifth prediction that the discrepancy between personal ideal body image and current body image is inversely correlated with female adolescents' satisfaction of their body image was examined. First, the discrepancies between current body image and ideal body image of the Figure Rating Scales was calculated. The discrepancy scores ranged from -2 to 3 with a mean difference of $.75(S D=.96)$. The mean on the 6 -item body image satisfaction scale of the Body Image/Media Survey was $17.55(S D=5.54)$ with a range from 6 to 30 .

Finally, a correlation was computed to determine whether there was a significant relationship between the difference of current body image and ideal body image with current body image satisfaction.

Results revealed that there was a statistically significant negative correlation with body image satisfaction and the discrepancy between current body image and ideal body image $(r=-.63, p<.001)$. This effect size was large (Cohen, 1988). Female adolescents who were more satisfied with their body image had a smaller discrepancy between their current body image and the personal ideal. 
Table 9 displays Pearson correlations that were computed to determine whether there were any relationships with body image and media influence measures and the discrepancy between current body image and ideal body image.

Table 9

Correlations of Current Body Image and Personal Ideal Discrepancy with Media Ideal Influence, SATAQ-3 subscales, Total SATAQ-3, and BMI

$$
\text { Current Body Image - Personal Ideal }
$$

Discrepancy

\begin{tabular}{ll}
\hline Body Image Satisfaction (BIMS) & $-.63^{\star \star \star}$ \\
Media Ideal Influence (BIMS) & .18 \\
Media Information (SATAQ-3) & .08 \\
Media Pressure (SATAQ-3) & $.37^{\star \star \star}$ \\
General Internalization (SATAQ-3) & $.29^{\star \star}$ \\
Athlete Internalization (SATAQ-3) & $.26^{\star}$ \\
Total SATAQ-3 & $.30^{\star \star}$ \\
BMI & $.60^{\star \star \star}$
\end{tabular}

Note. $n=107 .{ }^{\star} p<.01 .{ }^{\star \star} p<.005 .{ }^{\star \star \star} p<.001$.

The discrepancy between current body image and ideal body image was positively correlated with media pressure $(r=.37)$, general internalization $(r=$ $.29)$, athlete internalization $(r=.26)$, and total media influence $(r=.30)$. The 
effect sizes for these correlations were medium (Cohen, 1988). Greater discrepancy between current body image and ideal body image was associated with perceptions of more media pressure to be thin, more internalizing of the thin ideal, more internalizing of the athletic ideal, and more overall media influence. The discrepancy between current body image and personal ideal was significantly correlated with body mass index $(r=.60)$, a large effect size (Cohen, 1988). Heavy female adolescents had a heavier current body image relative to a heavy personal ideal.

\section{Prediction 6}

The sixth prediction, female adolescents perceive that the media ideal body image is thinner than their current body image, was examined. The frequencies of the discrepancy scores between current body image and media ideal body image revealed that $94 \%$ (102) of the participants indicated that the media ideal body image is thinner than their current body image. A dependent $t$ test was conducted to test for significance. Results from the $t$-test showed that the media ideal body image $(M=2.02, S D=.74)$ was significantly thinner than the current body image $(M=4.29, S D=1.20), t(106)=18.12, p<.001$. The media ideal was considerably thinner than the current body image, a mean difference of $2.27(S D=1.30)$. The effect size was large $(d=1.75$; Cohen, 1988). The current body image was not statistically significant with the media ideal $(r=.17, p=.089)$. Female adolescents' body image had little association with the media ideal. 


\section{Prediction 7}

The seventh prediction that the discrepancy between media ideal body image and current body image is inversely correlated with female adolescents' satisfaction of their body image was tested. The discrepancy between current body image and media ideal body image of the Figure Rating Scales ranged from -2 to 5 with a mean difference of $2.27(S D=1.30)$. On average, the current body image was heavier than the media ideal. In order to assess whether there was a linear relationship between discrepancy and satisfaction, the Body Image/Media Survey (BIMS) scales of body image satisfaction and media ideal influence were used along with the subscales (information, pressure, general internalization, and athlete internalization) and total scale of the Sociocultural Attitudes Towards Appearance Questionnaire-3 (SATAQ-3). Correlations were computed to discover whether there was a significant relationship between the female adolescent's satisfaction of their current body image and the discrepancy between their current body image and media ideal body image. Table 10 displays the intercorrelations of the BIMS, SATAQ-3 subscales and total scale, and BMI with the current body image and media ideal body image discrepancy and body image satisfaction scale. 
Table 10

Intercorrelations of the Current Body Image and Media Ideal Body Image

Discrepancy, Body Image Satisfaction, Media Ideal Influence, SATAQ-3

subscales, Total SATAQ-3, and BMI

\begin{tabular}{cc}
\hline Current Body Image - & Body Image \\
Media Ideal & Satisfaction \\
Discrepancy &
\end{tabular}

\begin{tabular}{lcc}
\hline Body Image Satisfaction (BIMS) & $-.47^{\star \star}$ & - \\
Media Ideal Influence (BIMS) & .04 & $-.36^{\star \star}$ \\
Media Information (SATAQ-3) & -.05 & -.02 \\
Media Pressure (SATAQ-3) & $.26^{\star}$ & $-.42^{\star \star}$ \\
General Internalization (SATAQ-3) & .14 & $-.35^{\star \star}$ \\
Athlete Internalization (SATAQ-3) & .05 & -.16 \\
Total SATAQ-3 & .12 & $-.28^{\star}$ \\
BMI & $.73^{\star \star}$ & $-.55^{\star \star}$ \\
\hline
\end{tabular}

Note. $n=107 .{ }^{\star} p<.01 .{ }^{\star \star} p<.001$.

The discrepancy between current body image and media ideal body image was negatively correlated with body image satisfaction $(r=-.47)$, a large effect size (Cohen, 1988). The discrepancy was positively correlated with media pressure $(r=.26)$; that is, the pressure from media to be thin. This effect size was medium (Cohen, 1988). Female adolescents who were heavy had a large discrepancy between current body image and media ideal body image, less body 
image satisfaction, and more felt pressure from media to be thin. The discrepancy between current body image and media ideal body image also was significantly correlated with the BMI ( $r=.73$ ), a large effect size (Cohen, 1988). Female adolescents who were heavy had a heavy BMI and a large discrepancy between current body image and media ideal body image.

In addition to being negatively correlated with the discrepancy between current body image and media ideal body image, body image satisfaction was negatively correlated with perceived pressure from the media $(r=-.42)$, internalization of the media ideal $(r=-.35)$, total media influence $(r=-.28)$, and media ideal influence $(r=-.36)$. The effect sizes for these correlations were medium (Cohen, 1988). Less body image satisfaction was associated with more perceived pressure from media to be thin, more internalization of the media ideal, more overall media influence (SATAQ-3), and more media ideal influence (BIMS). Body image satisfaction was also negatively correlated with BMI ( $r=$ $.55)$, a large effect size (Cohen, 1988). That is, female adolescents who were thin had a thin BMI and greater satisfaction with their body image.

The final purpose of this study was to determine whether there was a "best set" of predictors of female adolescents' satisfaction with their bodies from the following: participants' current and ideal body images, their BMI, their perception of the media ideal, the discrepancy between their current body image and ideal, the discrepancy between their current body image and media ideal, discrepancy between personal ideal and media ideal, their perception of whether they heard the message of thinness from media, the degree to which they 
internalized the thin ideal and athletic ideal, their perception of whether they felt pressure from media to be thin, and were influenced by it. A stepwise multiple regression analysis was conducted to predict body image satisfaction from these measures. The first predictor of body image satisfaction was the discrepancy between current body image and personal ideal body image. The correlation of the discrepancy between current body image and personal ideal was -.629 . This accounted for a statistically significant amount of body image satisfaction variance, $R^{2}=.40$, adjusted $R^{2}=.39, F(1,105)=68.64, p<.001$. The second predictor that entered was the media ideal influence. This increased the correlation from -.629 to -.674 and the $R^{2}$ from .40 to .46 , a statistically significant increase $\left(R^{2}\right.$ change $\left.=.06, F[1,104]=11.31, p=.001\right)$. The third predictor that entered was BMI. This increased the correlation to -.725 and the $R^{2}$ to .53 for an $R^{2}$ change $=.07, F(1,103)=15.29, p<.001$. Three factors accounted for fiftythree percent of the variance in body image satisfaction. When female adolescents had a greater discrepancy between their body image and personal ideal, more influence of the media ideal, and a heavier BMI, the less satisfied they were with their bodies.

\section{Prediction 8}

To test the eighth prediction that female adolescents will rate media as the strongest when asked to rank the relative influence of several sociocultural agents, the five most prevalent sociocultural factors that influence female's ideas about their bodies were presented to the participants on the Body Image/Media Survey. The sociocultural factors were family/guardians, peers, self, media, and 
males. These five factors were ranked from 1 - "most important" to 5 - "least important." In this sample, there was one participant who did not rank the sociocultural factors. Table 11 exhibits the means and standard deviations for the five sociocultural factors in order of importance.

Table 11

Means and Standard Deviations of the Sociocultural Factors in Rank Order

\begin{tabular}{lll}
\hline Sociocultural Factors & $M$ & $S D$ \\
\hline 1. Self & 2.23 & 1.42 \\
2. Family/Guardians & 3.00 & 1.44 \\
3. Peers & 3.01 & 1.01 \\
4. Males & 3.20 & 1.14 \\
5. Media & 3.57 & 1.45 \\
\hline
\end{tabular}

Note. $n=107$.

Frequencies of the "most important" ranking with each sociocultural factor were $44 \%$ (48) self, $13 \%$ (14) family/guardians, $12 \%$ (13) media, $7 \%$ (8) males, and $6 \%(6)$ peers. "Self" was ranked the most important influence of participants' ideas about their body images while "media" was the least important one. Thirtynine participants (36\%) ranked media as the least important influence. When asked how do females in media influence how you feel about your body, $26 \%$ (28) of the participants responded that media did not have any influence. Media was ranked as exerting the least important influence on body image. 


\section{Prediction 9}

This ninth prediction that female adolescents who rank media as a more important influence on their ideas about their bodies are less satisfied with their body image than those who rank media as less important was examined. A correlation was computed to test for significance of the relationship between body image satisfaction $(M=17.55)$ and media ranking $(M=3.57)$. The correlation was $.02(p=.88)$. There was no linear relationship between body image satisfaction and media ranking. 


\section{CHAPTER 5}

\section{DISCUSSION}

Research based on the sociocultural theory has shown that cultural and social experiences influence a person's perceptions. To what extent they do so is dependent on how relevant those values and standards are to the person. Researchers have demonstrated that perceptions of body images are susceptible to sociocultural influences. Direct experiential factors, like family and peers, as well as vicarious experiential factors, like media, have been shown to influence female adolescents' perceptions of their body image. The effect of media on female adolescents' perceptions of their body image was examined in the present study. Participants divulged their perceptions of current body image, personal ideal body image, and media ideal body image. This study also probed the relationship between media ideal body image and personal ideal body image. The association between actual body image and personal ideal and the influence of the media ideal on participants' current body image also were investigated. Finally, sociocultural factors were examined to determine their importance as an influence on participants' perceptions of their body image.

Prior to addressing the results, it is important to consider whether the participants are typical of young women in college. Although similar in most ways to college women in other studies, the participants of the present study 
weighed higher than average on the Body Mass Index (BMI). In fact, $43 \%$ of them exceeded the norms. Many body image researchers have reported BMI averages in the normal range for their respondents (Cattarin et al., 2000; Fingeret, Gleaves, \& Pearson, 2004; Morrison et al., 2004). Yet, according to the Centers for Disease Control and Prevention (2006), the mean BMI for women over 19 years old in America is 26.5 , clearly overweight by today's standards. Thus, the BMls of the participants in the present study are representative of the female population in America.

In summary, participants believed that both the media and personal ideal body images are thin. Participants' perceived body images were heavier than their ideal images. Most wanted to be thinner, and more participants were dissatisfied than satisfied with their bodies. They were especially displeased with their overall weight and specific body aspects. For these individuals, direct experiences were more influential in shaping their body image perceptions than vicarious experiences. Media did not appear to influence participants' satisfaction with their body image. Each of these findings will be discussed in more detail in their respective section: (a) ideal body images (Predictions 1, 2, and 3); (b) female adolescents' body image and interactions with ideal body images (Predictions 4 through 7); and (c) sociocultural factors (Predictions 8 and 9).

\section{Ideal Body Images}

Do female adolescents perceive the media ideal body image as thin? Using the thinness classification recommended by Bulik and associates (2001), 
all of the participants rated the media ideal body image as thin. This was reiterated by over half of them in their response to what was the media's most powerful message about the female body. A response by a 20 -year-old was typical, "Be thin! Almost all TV shows, commercials, and magazine ads show women who are impossibly thin." Thus, the findings support the first prediction of the study. Others have documented this thin ideal through analyses of models' measurements. These studies found that the media ideal is thin, especially when compared to the past (Garner et al., 1980; Guillen \& Barr, 1994; Silverstein et al., 1986; Wiseman et al., 1992). Although there are no current studies, these authors pointed out that women who look at media are exposed to a thinner ideal than in the past.

Personal ideal body image is defined by how young women desire to look. According to Bulik and associates' thinness classification (2001), most rated their personal ideal body image as thin. The mean rating of the personal ideal was slightly below the optimal cutoff of the thinness classification. That is, the heavier participants of this study were above the norms of the BMI. This finding supports the second prediction that female adolescents perceived their personal ideal as thin. The present study compared the mean personal ideal body image with the recommended thinness classification of the Figure Rating Scale. The Figure Rating Scale was designed as a continuum that ranged from extremely thin to extremely obese with figure 5 as the midpoint. It has been assumed that figures below the midpoint (figure 5) were thin figures. Bulik and associates (2001) have now quantified the classification of thinness on the Figure Rating Scale. The 
present study relied on their external standard to support the claim that the personal ideal body image is thin.

With the ideal body images established as thin, is the personal ideal related to the media ideal? Results, in general, support the third prediction of the study. Although not large, there was a moderate (according to Cohen's correlation convention; 1988), significant correlation between these two ideal body images $(r=.27, p<.01)$. When the personal ideal was thinner, the media ideal tended to be as well. Although correlated, the two ideal images did not have the same means. Most of the participants selected a media ideal thinner than their personal ideal. Cross tabulations also showed that the media ideal was thinner than the personal ideal at both high and low ends of the figure ratings on the Figure Rating Scale. In fact, $39 \%$ of the participants' personal ideal was only one figure away from the media ideal. Yet, the media ideal was significantly thinner than the personal ideal. The size of the mean difference was large, $1.51(S D=.90)$, thus the personal ideal usually was heavier relative to the media ideal.

It also is interesting to find that the discrepancy between media ideal and personal ideal was moderately correlated with the BMI. Individually, the media ideal was not linearly correlated with the BMI $(r=.04)$, but the personal ideal was. Therefore, the personal ideal was aligned with the BMI while the media ideal was not. Heavier participants maintained a heavier personal ideal relative to their media ideal. The personal ideal also had a small negative correlation with media as an information source of the thin ideal, internalization of the thin 
ideal, and internalization of the athletic ideal. When the personal ideal was thin, media were a source of information about the thin ideal and athletic ideal, and these ideals were internalized.

What do the findings of the ideal body images reveal? The large mean difference between the two ideal images indicated that the personal ideal was a considerably heavier figure than the media ideal. According to body image literature, the media ideal is thinner than the personal ideal (Fallon, 1990; Grogan, 1999; Monteath \& McCabe, 1997; Thompson et al., 1999; Turner et al., 1997). Not only does this finding confirm body image literature, but extends it by determining that the personal ideal was notably heavier relative to the media ideal. "Notably" is considered greater than one figure away. To illustrate, several participants selected figure 4 for their personal ideal and figure 2 for the media ideal. The measures defined two distinct ideal body shapes, one thin and one very thin. The personal ideal and media ideal, however, were moderately correlated. When the personal ideal increased in shape, the media ideal increased as well. Heavier participants usually exhibited a larger gap between their personal ideal and media ideal.

Generally, participants with a thin personal ideal internalized the media message of the thin ideal or athletic ideal. The thin or athletic ideal portrayed by media, however, were less influential with heavier participants who had a heavier personal ideal relative to the media ideal. The findings suggest that when the personal ideal and the media ideal were similar in body shape, participants internalized the message of the thin or athletic ideal from media. 
Body Image and Interactions with Ideal Body Images

Current body image is defined by how female adolescents mentally picture their bodies. A realistic perception of current body image is closely associated with body mass, a measure of weight for height. A number of investigations, however, found that females tend to overestimate or underestimate their body shape (Grogan, 1999; Monteath \& McCabe, 1997; Myers et al., 1992). In the present study, current body image was substantially correlated with the Body Mass Index (weight/height ${ }^{2}$ ). The magnitude of the effect was large. In fact, twothirds of the variance was attributed to the BMI. Many of the participants selected a current body image that closely resembled their actual body shape. Participants' current body image also was significantly correlated with body image satisfaction and moderately correlated with pressure from media to be thin. That is, participants who were thin had a thin body image, felt less pressure from the media to be thin, and were more satisfied with their bodies.

\section{Current Body Image and Personal Ideal}

What is the relationship between current body image and personal ideal body image? Results indicate that there was a significant relationship between current body image and personal ideal. When the current body image was heavy, the personal ideal was heavy. In other words, participants' personal ideal images were closely related to their current body images. Actual body shape also tended to be correlated with both the current body image and personal ideal. That is, heavy participants tended to have both a heavy current body image and a heavy personal ideal. For example, several participants, who were overweight 
according to the BMI, selected figure 5 on the Figure Rating Scale (FRS) for their current body image and figure 4 for their personal ideal. When the current body image and personal ideal were not close in shape, there was a distinct difference between the body images. In support of this claim, the discrepancy between current body image and personal ideal also was significantly correlated with BMI. Hence, heavy participants maintained a heavier body image relative to a heavy personal ideal. The personal ideal was aligned with the current body image. In general, the personal ideal was usually thinner, but some were the same or heavier, than the actual body image. Almost a third of the participants liked their body image, while over half wanted to be thinner, and a few wanted to be heavier.

Are female adolescents dissatisfied with their body image? This study predicted that they are dissatisfied with their body image. Two measures were used to analyze body image satisfaction. Discrepancy scores from the Figure Rating Scale determined that participants were dissatisfied with their body image. The personal ideal body image was significantly thinner than the participants' actual body image. The size of the mean difference was large, .75 (SD = .96). Over two-thirds of the participants were dissatisfied with their body image. More participants were dissatisfied than satisfied with their body image. This result replicates findings from previous studies using the Figure Rating Scale (Bulik et al., 2001; Fingeret et al., 2004; Morrison et al., 2004; Thompson et al., 1999).

A different distribution of dissatisfaction was demonstrated from the Body Image Satisfaction scale of the Body Image/Media Survey. The midpoint of the 
measure represented a neutral viewpoint, and all scores below it signified dissatisfaction. Fifty-one percent of the respondents were dissatisfied with their body image while $42 \%$ were satisfied. This scale also differed from the Figure Rating Scale in that the items on the body image satisfaction scale directly addressed body shape, weight, and body sites. Clear patterns emerged when these items were analyzed. The first item directly assessed satisfaction with one's body shape. Almost half of the participants were satisfied with their body shape, whereas a third were dissatisfied. When these participants were satisfied with their body shape, they generally were satisfied with their weight. Most of the participants who were dissatisfied with their body shape were dissatisfied with their weight. Many studies found that females who are not satisfied with their weight are generally dissatisfied with their body image (Dunkley, 2001; Garner, 1997; Grogan, 1999; Posavac et al., 1998; Turner et al., 1997; Wertheim et al., 1997). Participants who were satisfied with their body shape usually were not on a diet, whereas those who were dissatisfied usually were on a diet or felt that they should have been. Many of the participants were displeased with the way they looked in a swimsuit and disliked the appearance of their stomach and hips regardless of their body shape evaluation. Research also found that areas associated with weight gain like stomach, hips, and waist usually generate dissatisfaction in women (Garner, 1997; Monteath \& McCabe, 1997; Thompson et al., 1999). Several participants were pleased with the way clothes fit, especially when they were satisfied with their body shape. Findings of the Body Image Satisfaction scale demonstrate that it not only assessed satisfaction of 
body image, but also specific aspects of body image. More participants were dissatisfied with their body image as well as specific aspects of their body !riage except body shape and how clothes fit.

The Figure Rating Scale (FRS) and Body Image Satisfaction (BIS) scair assessed affective and cognitive aspects of body image. Truby and raxtor. (2002, p. 186) conveyed that the figure rating scale is "less abstract aro ieds:s less verbal fluency to comprehend than questionnaires." Specificall, tre $\mathrm{fH}$ relied on visual interpretations, whereas the BIS relied on textual internstit: The FRS was less threatening than the BIS, which was more senstiv $=$ if feelings. For instance, social desirability (i.e., My friends are dietirg treste...... should be dieting.) may have influenced the BIS responses more so that FRS responses. These scales offered two perspectives of the respond perceptions of their body image. The FRS rating consisted of extremely the extremely obese figures, whereas the BIS rating consisted of direct statem. about the body rated on a 5-point scale of strongly disagree to strongiv acras particular, more participants were satisfied with their bodies on the than the FRS. This result was attributed to the evaluation of severa! $a, \ldots$ body image on the BIS scale compared to the visual evaluation of twe images on the FRS. On the whole, more participants were dissatisfied wit: thet? bodies on both the FRS and BIS scale. Through the collective renderings the FRS and BIS scale, the findings, in general, support the fifth prediction that female adolescents are dissatisfied with their body image. 
When addressing the relationship between body image satisfaction and the discrepancy between current body image and personal ideal, results reveal a large, significant inverse correlation between these two scales $(r=-.63, p<$ .001). Hence, the greater the discrepancy, the less satisfied participants were with their bodies. When cross tabulations of the Figure Rating Scale and Body Image Satisfaction scale were analyzed to determine body image evaluation, clear patterns emerged that supported this result. Satisfaction patterns were identified when participants' scores yielded satisfaction with their body image on both the Figure Rating Scale and Body Image Satisfaction scale. Results of the satisfaction patterns indicate that $24 \%$ of the participants were satisfied with their body image. Likewise, dissatisfaction patterns were identified when participants' scores established dissatisfaction with their bodies on both scales. The results of the dissatisfaction patterns indicate that $46 \%$ of the participants were dissatisfied with their body image. In all, $70 \%$ of the participants were identified as either satisfied or dissatisfied with their bodies on both scales. It was predicted that female adolescents' satisfaction with their body image is inversely correlated with the discrepancy between participants' body image and their personal ideal. The present findings support this sixth prediction.

Although several analyses of the study revealed that the personal ideal closely resembled the current body image, many participants wanted to change their body shapes. In fact, many wanted a thinner body than they presently have. This result replicates findings from previous body image studies (Bulik et al., 2001; Fingeret et al., 2004; Grogan, 1999; Monteath \& McCabe, 1997; 
Thompson et al., 1999). Other investigators found that most women are dissatisfied with their bodies (Garner, 1997; Grogan, 1999; Monteath \& McCabe, 1997; Posavac et al., 1998). In this study, almost half of the participants were dissatisfied. This finding is a result of the collective analyses of two distinct satisfaction measures, the Figure Rating Scale (visual scale) and the Body Image Satisfaction scale (textual scale), that provided a more refined assessment of body image evaluation.

Results of the body image evaluation on the Body Image Satisfaction scale, in general, support the results of the Figure Rating Scale as well as provide details on specific aspects of the body. Grogan (1999) concluded that questionnaires offer a more detailed assessment of satisfaction with various aspects of the body. In the present study, many participants were satisfied with their body shape especially when it was clothed. When dissatisfied with their body shape, participants were definitely dissatisfied with their weight. In general, they were particularly dissatisfied with their stomach and hips especially when wearing a swimsuit.

As identified by both of the body image satisfaction scales, many participants were either satisfied or dissatisfied with their bodies. Participants whose current body image was similar to their personal ideal generally were satisfied with their body image, whereas participants whose current body image was heavier than their personal ideal generally were dissatisfied. This finding is consistent with the self-ideal discrepancy, that is the discrepancy between perceived body image and the personal ideal constitutes body dissatisfaction 
(Bulik et al., 2001; Fingeret et al., 2004; Morrison et al., 2004; Thompson et al., 1999).

Additional analyses of the discrepancy between current body image and personal ideal identified particular aspects of body image that were influenced by media. This discrepancy was moderately correlated with media pressure to be thin, general internalization of the thin ideal, internalization of the athletic ideal, and total media influence. Findings suggest that media, especially the pressure from media to be thin and internalization of the thin or athletic ideal, influenced participants whose body image was notably heavier than their personal ideal and were dissatisfied with their bodies.

\section{Current Body Image and Media Ideal Body Image}

Do female adolescents perceive the media ideal body image as thinner than their current body image? All but five of the respondents indicated that the media ideal body image was thinner than their current body image. This result supports the sixth prediction that female adolescents perceive the media ideal as thinner than their actual body image. This finding replicates conclusions from prior investigations comparing current body image and media ideal (Garner et al., 1980; Grogan, 1999; Monteath \& McCabe, 1997; Morrison et al., 2004; Thompson et al., 1999; Turner et al., 1997). In fact, the media ideal was considerably thinner than the current body image. The size of the mean difference was large, $2.27(S D=1.30)$, suggesting that there was little association between current body image and the media ideal. There was no statistical significance between these two measures $(r=.17, p=.089)$. 
Interestingly, there was no evidence of a relationship between participants' body image and the media ideal.

Findings also support the seventh prediction that the discrepancy between current body image and media ideal body image is inversely correlated with female adolescents' satisfaction of their body image. A large, significant negative correlation was determined between body image satisfaction and the discrepancy between current body image and media ideal $(r=-.47, p<.001)$. That is, the greater the discrepancy between body image and the media ideal, the more dissatisfied the participants were with their bodies. All but two of the participants exhibited a discrepancy between their current body image and the media ideal on the Figure Rating Scale. When analyzing cross tabulations of the Figure Rating Scale and Body Image Satisfaction scale to determine body image satisfaction, patterns emerged that supported this result. Fifty-two percent of the participants were identified as either satisfied or dissatisfied with their bodies on both the Figure Rating Scale and Body Image Satisfaction scale. Results of the satisfaction patterns indicate that $2 \%$ of the participants were satisfied with their body image. Whereas, the results of the dissatisfaction patterns indicate that half of the participants were dissatisfied with their body image.

Participants' body images were substantially heavier than the media ideal. Current body image ranged from 2 to 8 on the Figure Rating Scale, whereas the media ideal ranged from 1 to 4 . Only two participants' body images resembled the media ideal. No discernible pattern was found between current body image 
and the media ideal. These findings verify that perceived body image and the media ideal were not related.

The relationship between body image satisfaction and the discrepancy of current body image with the media ideal was strong. Distributions of both satisfaction and dissatisfaction discrepancy scores were skewed. In fact, only two participants were satisfied with their body image, whereas the rest were dissatisfied according to the results of the Figure Rating Scale. Body dissatisfaction was prominent when analyzing the self-media ideal discrepancy. Monteath \& McCabe (1997) found that larger discrepancies from the societal ideal are correlated with higher levels of body dissatisfaction. At face value this may be true, however, cross tabulations of two body image satisfaction scales provided a different perspective of the dissatisfaction distribution. Half of the participants were dissatisfied with their bodies when the body image satisfaction scale was factored into the analysis. Even though several participants were satisfied with their bodies on the body image satisfaction scale, only two participants' body image were the same as the media ideal on the Figure Rating Scale. Using the media ideal in a self-ideal discrepancy produces skewed results, because many of the participants' current body images were not close to the media ideal. Large discrepancies also suggest that media influenced a greater number of participants.

In spite of this, results show that over half of the participants conveyed either satisfaction or dissatisfaction with their bodies on both the FRS and BIS. Participants whose current body image was similar to the media ideal generally 
were satisfied with their body image, whereas participants whose current body image was heavier relative to the media ideal generally were dissatisfied. This finding replicates Monteath and McCabe's (1997) result that larger discrepancies of women's actual body size to the societal ideal are related to body dissatisfaction.

Additional analyses of the discrepancy between current body image and media ideal identified particular aspects of body image that were influenced by media. This discrepancy was significantly correlated with BMI and moderately correlated with media pressure. In other words, heavy participants, who had a heavy body image but a thin media ideal, felt more pressure from media to be thin and were dissatisfied with their bodies. This finding accords well with Garner's (1997) conclusion that media influence most strongly women who are distinctly heavier than their cultural ideal and dissatisfied with their shape.

\section{Predictors of Body Image Satisfaction}

A stepwise multiple regression analysis was conducted with body image satisfaction as a criterion. Variables of body images, discrepancies, BMI, SATAQ-3, and media ideal influence scale were entered to designate the "best set" of predictors of body image satisfaction. The three best predictors of body image satisfaction were the discrepancy between current body image and personal ideal body image, media ideal influence, and BMI. In fact, $53 \%$ of the variance in body image satisfaction was accounted for by these three factors alone. 
The discrepancy between current body image and personal ideal accounted for most of the variance in body image satisfaction. Of the three predictors, discrepancy scores were significantly correlated with body image satisfaction. The greater the discrepancy between current body image and personal ideal, the less satisfied participants were with their bodies. As discussed previously, this finding confirms previous studies' conclusions that the self-personal ideal discrepancy indicates body satisfaction (Bulik et al., 2001; Fingeret et al., 2004; Morrison et al., 2004; Thompson et al., 1999). In fact, the self-personal ideal discrepancy exhibited a stronger relationship with body image satisfaction than the self-media ideal discrepancy. The self-personal ideal discrepancy revealed that $70 \%$ of the participants conveyed either satisfaction or dissatisfaction with their bodies, whereas only $52 \%$ conveyed this with the selfmedia ideal discrepancy. The self-media ideal discrepancy scores identified only two participants satisfied with their bodies, while the self-personal ideal discrepancy scores identified a third satisfied. Furthermore, a significant relationship between current body image and the personal ideal was found, while no evidence of a relationship between current body image and the media ideal was ascertained. The findings indicate that the personal ideal is the better ideal image to use in body image evaluation. Jacobi and Cash (1994) expressed that ideals shaped from one's own perceptions are more germane in body image evaluation, a conclusion clearly demonstrated in the present study.

Influence of the media ideal was the second entered predictor of body image satisfaction. The media ideal influence scale assessed the influence of 
women from different mediums on how participants would like to look. The media ideal influence was moderately correlated with body image satisfaction. In general, participants who agreed that they were influenced by the media ideal were less satisfied with their bodies, whereas participants who disagreed were more satisfied. This finding supports prior studies that found women who are influenced by media are dissatisfied with their bodies (Monteath \& McCabe, 1997; Tiggemann \& Pickering, 1996; Turner et al., 1997). In combination with both the Figure Rating Scale and the Body Image Satisfaction scale, only a small cohort of participants were identified as influenced by the media ideal and dissatisfied with their bodies. These participants perceived that the thin ideal portrayed by media contributed to their body dissatisfaction.

BMI was also a predictor of body image satisfaction. As established in this study, BMI was significantly correlated with current body image, personal ideal body image, and their discrepancies. Body dissatisfaction usually was attributed to a heavy BMI, whereas body satisfaction usually was attributed to a thin BMI. This result is consistent with Monteath and McCabe's (1997) finding that increasing BMI is correlated with a higher level of body dissatisfaction.

The combined influence of these three measures predicted body image satisfaction better than any one measure. The present findings suggest that heavy participants, who had a heavier body image relative to their personal ideal and were influenced by the media ideal, were dissatisfied with their body image. 


\section{Sociocultural Factors}

According to the literature, a number of sociocultural factors influence young women's perceptions about their body image (Dunkley et al., 2001; Grogan, 1999; Levine et al., 1994; Thompson et al., 1999; van den Berg et al., 2002). The five sociocultural factors assessed by this study were family/guardians, peers, males, self, and media. Over the years, researchers have used rating scales to assess the importance of sociocultural factors' influences (Dunkley et al., 2001; Irving, 1990; Thompson et al., 1999). The consensus from many of these studies is that sociocultural factors are important influences on body image perceptions. However, studies that depended on rating scales have not revealed the relative influence of each factor. For example, ratings cannot determine whether media are more influential than peers. In the present study, participants were asked to rank sociocultural factors so their order of importance would be ascertained.

This study predicted that female adolescents would rate media as the strongest influence of several sociocultural factors. Sociocultural factors were ranked in order of most to least influential in shaping participants' body image: self, family/guardians, peers, males, and media. Participants rated self as the strongest influence in shaping their body image perceptions. It is not surprising that participants selected self as the most important influence at this developmental phase. The evolving self generally is the focal point during the identity critical period in late adolescence. Female adolescents are coming to terms with their identity especially the physical self. They are autonomous and 
rely on their sense of self at this stage in their lives (Berk, 2004; Jackson, 1992; Pipher 1994). It is surprising that family was ranked second especially with college women. Family members are influential throughout young women's lives; however, late adolescence is predominately a time of strong peer relationships (Berk, 2004; Brumberg, 1997; Pipher 1994). Researchers generally have found that peers are more influential with their own age group regarding ideas about body image during adolescence (Grogan, 1999; Jackson, 1992; Pipher, 1994; Thompson et al., 1999). Nevertheless, family being selected as the second most important influence may be attributed to many of the participants living at home, where they still have everyday interactions with family members.

Results do not support the eighth prediction that female adolescents rate media the strongest when ranking the relative influences of sociocultural factors. Media were the least important influence on these participants' perceptions of their body image. Over half indicated that media were less or least influential of the sociocultural factors. Several participants indicated that media did not have any influence on how they felt about their bodies. Why did media have little influence on many of the participants? Many perceived that the media ideal was too thin. A response by a 19-year-old was typical, "They are too skinny and I wouldn't want to be that small." Many participants also felt that it was unrealistic. The media ideal was not representative of their thin ideal. Media were least influential of these sociocultural factors for this age group.

Nevertheless, a small cohort of participants identified media as the most important influence. These participants perceived media as very influential in 
shaping their body image. Their perceptions aligned with the results of numerous studies defining media as an important influence on young women's perceptions of body image (Dunkley et al., 2001; Grogan, 1999; Hargreaves \& Tiggemann, 2003; Monteath \& McCabe, 1997; Tiggemann et al., 2000; Wertheim et al., 1997).

The findings also do not support the ninth prediction that female adolescents who rank media as an important influence in shaping their body image are less satisfied with their bodies. No linear correlation was determined between media rank and body image satisfaction. No evidence of a relationship was found. Findings indicate that media influence in participants' perceptions of their body image was unrelated to their satisfaction with their bodies. This result disconfirms Monteath and McCabe's (1997) finding that many women are influenced by the societal ideal, and it affects how women feel about their bodies. In fact, none of the other sociocultural factors were correlated with body image satisfaction. One measure assesses influence on body image perceptions, while the other evaluates body image satisfaction. Sociocultural factors are influential in shaping body image perceptions, yet they are not overtly influential in the personal assessment of body image evaluation. As previously discussed, body image evaluation warrants the interactions of a few variables especially the discrepancy between body image and personal ideal. Participants generally perceived that the media ideal had little influence in shaping their personal body image and did not determine their satisfaction with their bodies. 


\section{Summary}

Female adolescents perceive that direct experiences with self, family, and friends are more influential in their perceptions of their body image than vicarious experiences with media. In general, they believe that media have little influence in shaping their body image.

Female adolescents perceive the media ideal as considerably thinner than their personal ideal. In fact, the media ideal is very thin, whereas the personal ideal is thin. They are two distinct ideal body shapes. Female adolescents are heavier than these ideal body images. Their current body image usually reflects their own body shape. Female adolescents' body images are closely related to their personal ideal images even though they are thinner. For example, female adolescents who are heavy also have a heavier body image relative to a heavy personal ideal. In general, they want a thinner body than they currently have. Female adolescents whose body image is heavier than their personal ideal generally are dissatisfied with their bodies, whereas female adolescents whose body image is similar to their personal ideal generally are satisfied.

Media generally influence female adolescents whose personal ideal images are notably thinner than their body images yet similar to the media ideal. These adolescents internalize the message from media to be thin, and then feel the pressure from media to be thin. Female adolescents who are influenced by the media ideal are dissatisfied with their bodies. In the present study, only a small group of participants indicated that they were influenced by media. 
In essence, media have little influence on female adolescents' perceptions of their body image. Hence, the thin ideal as portrayed by media has little influence on female adolescents' personal ideal. In fact, female adolescents do not perceive the media ideal as representative of their thin ideal. As a 19-yearold expressed, "They are too skinny." Female adolescents want to be thinner but not as thin as the media ideal. Another 19-year-old conveyed this group of participants' sentiments, "Trying to look like a media model is unrealistic." On the whole, the very thin ideal portrayed by media generally does not influence female adolescents' satisfaction with their body image.

\section{Limitations}

This study has several limitations. First, mailing surveys to all female students at the recruited colleges guaranteed reaching more females than recruiting on site. Of course, there was no guarantee of the return rate with mailings. Personal contact was important to the researcher in case recruits had questions or offered suggestions about the study. The researcher relied on the "Young Women's Body Image Study" sign to recruit young women at the designated college campuses. Surveys were distributed to a convenient sample of females who approached the area. These volunteers were motivated to participate. This may have produced a biased sample, because young women who were too shy or not at that particular location on campus at the time were missed. By handing out surveys on site, the researcher may have missed including a larger, more diverse group of young women. 
A second limitation of this study pertains to procedural protocol. In order to exert some control over the settings, the researcher recited scripted instructions to the volunteers when distributing the survey packets. At various times during the study, several young women wanted to participate within minutes of each other. The researcher condensed the scripted instructions to accommodate them. In addition, a faculty member from the second branch of the career college conducted the study at her site. Even though the researcher went through the procedures with the faculty member, she was unable to control the setting and relied on the faculty member to follow procedural protocol. No visual images, like the body image study sign, were present to engage volunteers at this location. Any combination of procedural inconsistencies may have affected response patterns.

Another limitation concerns instrumentation. This study was the initial application of the Body Image/Media Survey (BIMS). Strong reliability estimates were determined for the body image satisfaction scale (.82) and media ideal influence scale (.88). With regards to the body appearance open-ended question, there were several responses that clearly did not answer the question. For future research, the question will be restated to make it clearer. In addition, almost a quarter of the participants repeated numbers in their ranking of sociocultural factors when the numbers should have been used only once. Averages were computed for these ranking responses. Further, the semantics of "most important influence" and "least important influence" may have biased the ranking. "Important" may have been considered relevant to direct experiences, 
such as self, family, peers, and males, and not as relevant to vicarious experiences, like media, for the participants. These descriptors will be changed to "most influential" and "least influential".

A final limitation concerns response bias. The Sociocultural Attitudes Towards Appearance Questionnaire-3 and the Body Image/Media Survey had reversed order items to control response bias. Directions to "select the best answer that reflects how you think and feel" also were given to the volunteers before they answered the surveys in order to minimize response bias. Participants, however, may have answered questions from a socially desirable response set. Some of the participants' responses may have been selected in order to seek approval from friends or the researcher. When filling in their surveys together, two or more of the participants may have collaborated on their responses.

\section{Future Research}

One criticism of several prior studies was that young women who do not attend college have been neglected in body image research. In fact, many of the previous body image studies with the same age cohorts were conducted on fouryear college campuses. This was most likely a result of researchers' convenience. In order to study a more representative group of young women in America, participants were drawn from two-year, four-year, and career colleges. Only $20 \%$ of the participants, however, represented career colleges, because of the limited number of females between 18-21 years old in attendance. Some of the participants represented the four-year college like the samples in other body 
image research. Future research needs to include a larger, more representative sample of female adolescents especially non-college bound females and those attending two-year and career colleges.

When evaluating the media ideal body image, there were interesting results that, in turn, elicited questions about this ideal. For example, the media ideal is a very thin ideal according to several studies (Garner et al., 1980; Silverstein et al., 1986; Wiseman et al., 1992) and the present study. Has the thin ideal portrayed by media become too thin? The media ideal also was considerably thinner than the personal ideal. In fact, they were two distinct ideal images. Why was there such a large discrepancy between two ideal images? Furthermore, no relationship was established between the media ideal and current body image. These findings led the researcher to question whether the media ideal mirrors the sociocultural norms. Body image literature has indicated that media reflect the sociocultural norms (Grogan, 1997; Jackson, 1992, 2002; Thompson et al., 1999; Tiggemann, 2002). Sociocultural norms are determined by culture and society as a whole. Yet, participants perceived that the media ideal was unrealistic, and had little influence on their perceptions of their personal ideal and actual body images. These participants are part of society; ergo, they should have some affect on sociocultural norms. Are the media ideal and the sociocultural ideal one in the same? Future studies could clarify whether the media ideal truly reflects the sociocultural norm of the ideal body image.

Interactions of the media ideal and personal ideal also produced some interesting results. A moderate relationship was determined between the media 
ideal and personal ideal, yet the media ideal was considerably thinner than the personal ideal. Results also indicated that the media ideal was not even representative of the participants' thin ideal images. Were these findings attributed to a heavier group of participants? Or were they a result of the age cohort? The relationship between the personal ideal and media ideal is an important area of media-body image research and needs further attention.

Prior research found that media are an important influence on body image perceptions (Grogan, 1999; Monteath \& McCabe, 1997; Tiggemann et al., 2000; Wertheim et al., 1997). Based on the sociocultural factors included in this study, media were determined to be the least important influence in shaping female adolescents' body image except for a small cohort. Other studies generally rated sociocultural factors, whereas this study ranked them. Ranking established an order to the sociocultural factors' importance in their influence on participants' body images. Yet, this ranking did not determine the absolute importance of each factor. Even though media were the least influential factor relative to the other sociocultural factors, were they still deemed important by the participants? This issue could be addressed by asking participants how important each factor's influence is to them. The ranking of sociocultural factors' influence on body image perceptions and their overall importance to the individual need further investigation.

A longitudinal approach also could explore the sociocultural factors' influence on perceptions of body image. For example, longitudinal research would reveal whether the influences of sociocultural factors vary 
developmentally. When assessing media, female adolescents' combined histories of exposure to media, media usage, and media's impact on body image would determine the cumulative effect of media influence. In brief, longitudinal research would identify whether there are patterns of media influence on body image.

Media exert influence on female adolescents whose personal ideal is considerably thinner than their body image, but similar to the media ideal, and are dissatisfied with their bodies. Thin participants do not fit this profile, yet media influence some. Are there particular aspects of body image of thin females that are influenced by media? Media influenced only a small cohort of participants in the present study. Why so few? Was this a function of the age group? Were fewer females swayed by the media ideal?

As demonstrated in this study and prior research, a complex association exists between media and body image. It is through research such as this study that we advance our knowledge of media influence. The present study identified several directions for future research of the influence of media on females' satisfaction with their body image. 


\section{REFERENCES}

Banner, L. (1983). American beauty. New York: Alfred A. Knopf.

Bear, G., Minke, K., \& Thomas A. (Eds.)(1997). Children's needs II: Development, problems, and altematives. Bethesda, MD: National Association of School Psychologists.

Berk, L. (2004). Development through the lifespan ( $3^{\text {rd }}$ ed.). Boston: Allyn and Bacon.

Bermudez, J., Marcel, A., \& Eilan, N. (Eds.) (1995). The body and the self. Cambridge, MA: The MIT Press.

Botta, R. (1999, Spring). Television images and adolescent girls' body image disturbance. Joumal of Communication, 49 (2), 22-41.

Botta, R. (2000, Summer). The mirror of television: A comparison of black and white adolescents' body image. Joumal of Communication, 50 (3), 144-159.

Borzekowski, D., Robinson, T., \& Killen, J. (2000). Does the camera add 10 pounds? Media use, perceived importance of appearance, and weight concerns among teenage girls. Joumal of Adolescent Health, 26 (1), 36-41.

Bracken, B. (Ed.) (1996). Handbook of self-concept: Development, social, and clinical considerations. New York: John Wiley \& Sons, Inc.

Brooks-Gunn, J. \& Reiter, E. (1990). The role of pubertal processes. In S. Feldman \& G. Elliott (Eds.), At the threshold: The developing adolescent (pp. 16-53). Cambridge, MA: Harvard University Press.

Brumberg, J. (1997). The body project: An intimate history of American girls. New York: Vintage Books.

Bulik, C., Wade, T., Heath, A., Martin, N., Stunkard, A., \& Eaves, L. (2001). Relating body mass index to figural stimuli: Population-based normative data for Caucasians. International Joumal of Obesity, 25, 1517-1524.

Buss, A. (2001). Psychological dimensions of the self. Thousand Oaks, CA: Sage Publications. 
Cash, T. (1990). The psychology of physical appearance: Aesthetics, attributes, and images. In T. Cash \& T. Pruzinsky (Eds.), Body images: Development, deviance, and change (pp. 51-79). New York: Guilford Press.

Cash, T. \& Fleming, E. (2002). Body image and social relations. In T. Cash \& T. Pruzansky (Eds.), Body image: A handbook of theory, research, and clinical practice (pp. 277-286). New York: Guilford Press.

Cash, T. \& Pruzinsky, T. (Eds.)(1990). Body images: Development, deviance, and change. New York: Guilford Press.

Cash, T. \& Pruzinsky, T. (Eds.)(2002). Body image: A handbook of theory, research, and clinical practice. New York: Guilford Press.

Cattarin, J., Thompson, J. K., Thomas, C., \& Williams, R. (2000). Body image, mood, and televised images of attractiveness: The role of social comparison. Joumal of Social and Clinical Psychology, 19 (2), 220-239.

Centers for Disease Control and Prevention (2004, December). BMI-Body Mass Index: BMI for adults: What does it mean? Retrieved October 2005, from http://unw.cdc.gov/nccdphp/dnpa/bmi

Centers for Disease Control and Prevention (2006, March). Healthy weight, overweight, and obesity among U.S. adults. Retrieved March 22, 2006, from http://unw.cdc.gov/nchs/data/nhanes/databriefs/adultweight.pdf

Cohen, J. (1988). Statistical power analysis for the behavioral sciences $\left(2^{\text {nd }}\right.$ ed.). Hillsdale, NJ: Lawrence Erlbaum Associates, Publishers.

Cole, D., Browning, E., \& Schroeder, F. (2003). Encyclopedia of modem everyday inventions. Westport, CT: Greenwood Press.

Collins, J. (1981). Self-recognition of the body and its parts during late adolescence. Joumal of Youth and Adolescence, 10 (3), 243-254.

Cooley, C. H. (1902). Human nature and the social order. New York: Charles Scribner's Sons.

Currie, D. (1999). Girl talk: Adolescents magazines and their readers. Toronto, Canada: University of Toronto Press.

Davies, E. \& Furnham, A. (1986). Body satisfaction in adolescent girls. British Joumal of Medical Psychology, 59, 279-287. 
Davis, R. (2004, July 29). Teens' cosmetic dreams don't always come true: Decisions on plastic surgery can be premature. USA Today, pp. D1, D2, D8.

Douglas, M. (1996). Natural symbols. London: Routledge.

Duncan, M., Dodd, L., \& Al-Nakeeb, Y. (2005). The impact of silhouette randomization on the results of figure rating scales. Measurement in Physical Education and Exercise Science, 9 (1), 61-66.

Dunkley, T., Wertheim, E., \& Paxton, S. (2001). Examination of a model of multiple sociocultural influences of adolescent girls' body dissatisfaction and dietary restraint. Adolescence, 36 (142), 265-279.

Durkin, S. \& Paxton, S. (2002). Predictors of vulnerability to reduced body image satisfaction and psychological wellbeing in response to exposure to idealized female media images in adolescent girls. Joumal of Psychosomatic Research, 53, 995-1005.

Etcoff, N. (1999). Survival of the prettiest: The science of beauty. New York: Doubleday.

Fallon, A. (1990). Culture in the mirror: Sociocultural determinants of body image. In T. Cash \& T. Pruzinsky (Eds.), Body images: Development, deviance, and change (pp. 80-109). New York: Guilford Press.

Feldman, S. \& Elliott, G. (Eds.)(1990). At the threshold: The developing adolescent. Cambridge, MA: Harvard University Press.

Ferron, C. (1997). Body image in adolescence: Cross-cultural research - results of the preliminary phase of a quantitative survey. Adolescence, 32 (127), 735745.

Field, A., Cheung, L., Wolf, A., Herzog, D., Gortmaker, S., \& Colditz, G. (1999). Exposure to the mass media and weight concerns among girls. Pediatrics, 103 (3), e36.

Fingeret, M., Gleaves, D., \& Pearson, C. (2004, January 7). On the methodology of body image assessment: The use of figural rating scales to evaluate body dissatisfaction and the ideal body standards of women. Body Image, 1(003), 207-212. Retrieved October 2005, from http://wuw.elsevier.com/locate/bodyimage

Fine, G., Mortimer, J., \& Roberts, D. (1990). Leisure, work, and the mass media. In S. Feldman \& G. Elliott (Eds.), At the threshold: The developing adolescent (pp. 225-252). Cambridge, MA: Harvard University Press. 
Fisher, S. (1986). Development and structure of the body image (Vol. 1). Hillsdale, NJ: Lawrence Erlbaum Associates, Publishers.

Garner, D. (1997, February). The 1997 body image survey results. Psychology Today, 1-13.

Garner, D., Garfinkel, P., Schwartz, D. \& Thompson, M. (1980). Cultural expectations of thinness in women. Psychological Reports, 47, 483-491.

Gimlin, D. (2002). Body work: Beauty and self-image in American culture. Los Angeles: University of California Press.

Grogan, S. (1999). Body image: Understanding body dissatisfaction in men, women, and children. New York: Routledge.

Guillen, E. \& Barr, S. (1994). Nutrition, dieting, and fitness messages in a magazine for adolescent women, 1970-1990. Joumal of Adolescent Health, $15,464-472$.

Haimovitz, D., Lansky, L., \& O' Reilly, P. (1993). Fluctuations in body satisfaction across situations. Intemational Joumal of Eating Disorders, 13 (1), 77-84.

Hargreaves, D. \& Tiggemann, M. (2003). Longer-term implications of responsiveness to 'thin-ideal' television: Support for a cumulative hypothesis of body image disturbance? European Eating Disorders Review, 11 (6), 465477.

Harter, S. (1990). Self and identity development. In S. Feldman \& G. Elliott (Eds.), At the threshold: The developing adolescent (pp. 352-387). Cambridge, MA: Harvard University Press.

Hesse-Biber, S. (1996). Am I thin enough yet? The cult of thinness and the commercialization of identity. New York: Oxford University Press.

Holmstrom, A. (2004). The effects of the media on body image: A meta-analysis. Joumal of Broadcasting \& Electronic Media, 48 (2), 196-217.

Irving, L. (1990). Mirror images: Effects of the standard of beauty on the selfand body-esteem of women exhibiting varying levels of bulimic symptoms. Joumal of Social and Clinical Psychology, 9 (2), 230-242.

Jackson, L. (1992). Physical appearance and gender: Sociobiological and sociocultural perspectives. New York: State University of New York Press. 
Jacobi, L. \& Cash, T. (1994). In pursuit of the perfect appearance: Discrepancies among self-ideal percepts of multiple physical attributes. Joumal of Applied Social Psychology, 24 (5), 379-396.

Jaffee, L. \& Lutter, J. (1995). Adolescent girls: Factors influencing low and high body image. Melpomene Joumal, 14 (2), 14-22.

Johnson, N., Roberts, M., \& Worell, J. (Eds.)(1999). Beyond appearance: A new look at adolescent girls. Washington, DC: American Psychological Association.

Kaplan, E. (1997). Women's perceptions of the adolescent experience. Adolescence, 32 (127), 715-734.

Kearnery- Cooke, A. (2002). Familial influences on body image development. . In T. Cash \& T. Pruzinsky (Eds.), Body image: A handbook of theory, research, and clinical practice (pp. 99-107). New York: Guilford Press.

Kitch, C. (2001). The girl on the magazine cover: The origins of visual stereotypes in American mass media. Chapel Hill, NC: The University of North Carolina Press.

Levine, M. \& Smolak, L. (2002). Body image development in adolescence. In T. Cash \& T. Pruzinsky (Eds.), Body image: A handbook of theory, research, and clinical practice (pp. 74-82). New York: Guilford Press.

Levine, M., Smolak, L., \& Hayden, H. (1994). The relation of sociocultural factors to eating attitudes and behaviors among middle school girls. Joumal of Early Adolescence, 14 (4), 471-490.

Levinson, R., Powell, B., \& Steelman, L. (1986). Social location, significant others and body image among adolescents. Social Psychology Quarterly, 49 (4), 330-337.

Maine, M. (2000). Body wars: Making peace with women's bodies. Carlsbad, CA: Gurze Books.

Mann, J. (1994). The difference: Growing up female in America. New York: Warner Books, Inc.

McCabe, M. \& Ricciardelli, L. (2003). Sociocultural influences on body image and body changes among adolescent boys and girls. Joumal of Social Psychology, 143 (1), 5-26.

Monteath, S. \& McCabe, M. (1997). The influence of societal factors on female body image. The Joumal of Social Psychology, 137 (6), 708-727. 
Morrison, T., Kalin, R., \& Morrison, M. (2004). Body-image evaluation and bodyimage investment among adolescents: $A$ test of sociocultural and social comparison theories. Adolescence, 39 (155), 571-592.

Murray, S., Touyz, S. \& Beumont, P. (1995). The influence of personal relationships on women's eating behavior and body satisfaction. Eating Disorders: The Joumal of Treatment and Prevention, 3, 243-252.

Must, A., Phillips, S., Stunkard, A., \& Naumova, E. (2002). Expert opinion on body mass index percentiles for figure drawings at menarche. Intermational Joumal of Obesity, 26, 876-879.

Myers, P. \& Biocca, F. (1992). The elastic body image: The effect of television advertising and programming on body image distortions in young women. Joumal of Communications, 42 (3), 108-133.

Neisser, U. (Ed.) (1993). The perceived self: Ecological and interpersonal sources of self-knowledge. Cambridge, England: Cambridge University Press.

Nichter, M. \& Vuckovic, N. (1994). Fat talk: Body image among adolescent girls. In N. Sault (Ed.), Many mirrors: Body image and social relations (pp. 109-131). New Brunswick, NJ: Rutgers University Press.

Paxton, S., Schutz, H., Wertheim, E., \& Muir, S. (1999). Friendship clique and peer influences on body concerns, dietary restraint, extreme weight-loss behaviors, and binge eating in adolescent girls. Joumal of Abnormal Psychology, 108 (2), 255-266.

Phelps, L., Augustyniak, K., Nelson, L., \& Nathanson, D. (1997). Adolescent eating disorders, chronic dieting, and body dissatisfaction. In G. Bear, K. Minke, \& A. Thomas (Eds.), Children's needs II: Development, problems, and altematives (pp. 909-915). Bethesda, MD: National Association of School Psychologists.

Pipher, M. (1994). Reviving Ophelia: Saving the selves of adolescent girls. New York: Ballantine Books.

Polce-Lynch, M., Myers, B., Kilmartin, C., Forssmann-Falck, R., \& Kliewer, W. (1998). Gender and age patterns in emotional expression, body image, and self-esteem: A qualitative analysis. Sex Roles, 38 (11/12), 1025-1048.

Posavac, H., Posavac, S., \& Posavac, E. (1998). Exposure to media images of female attractiveness and concern with body weight among young women. Sex Roles, 38 (3/4), 1-12. 
Pruzinsky, T. \& Cash, T. (2002). Understanding body images: Historical and contemporary perspectives. In T. Cash \& T. Pruzinsky (Eds.), Body image: A handbook of theory, research, and clinical practice (pp. 3-12). New York: Guilford Press.

Rieves, L. \& Cash, T. (1996). Social developmental factors and women's bodyimage attitudes. Joumal of Social Behavior and Personality, 11, 63-78.

Rosenblum, G. \& Lewis, M., (1999). The relations among body image, physical attractiveness, and body mass in adolescence. Child Development, 70 (1), 50-64.

Sanftner, J., Crowther, J., Crawford, P. \& Watts, D. (1996). Maternal influences (or lack thereof) on daughters' eating attitudes and behavior. Eating Disorders: The Joumal of Treatment and Prevention, 4, 147-159.

Sault, N. (Ed.) (1994). Many mirrors: Body image and social relations. New Brunswick, NJ: Rutgers University Press.

Sault, N. (1994). Introduction: The human mirror. In N. Sault (Ed.), Many mirrors: Body image and social relations (pp. 1-28). New Brunswick, NJ: Rutgers University Press.

Schilder, P. (1950). The image and appearance of the human body: Studies in the constructive energies of the psyche. New York: International Universities Press, Inc.

Schwartz, M. \& Brownell, K. (2002). Obesity and body image. In T. Cash \& T. Pruzinsky (Eds.), Body image: A handbook of theory, research, and clinical practice (pp. 200-209). New York: Guilford Press.

Shontz, F. (1993). The ecological self in historical context. In U. Neisser (Ed.), The perceived self: Ecological and interpersonal sources of self-knowledge (pp. 89-101). Cambridge, England: Cambridge University Press.

Signorelli, N. (1997, April). A content analysis: Reflections of girls in the media (Publication No. 1260). Retrieved October 2004, from the Kaiser Family Foundation Web site: http://www.kff.org/entmedia/1260-gendr.cfm

Silverstein, B., Perdue, L., Peterson, B., \& Kelly, E. (1986). The roles of the mass media in promoting a thin standard of bodily attractiveness for women. Sex Roles, 14 (9/10), 519-532.

Singer, D. \& Singer, J. (2001). Introduction: Why a handbook on children and the media? In D. Singer \& J. Singer (Eds.), Handbook of children and the media (pp. xi-xvii). Thousand Oaks, CA: Sage Publications. 
Singer, D. \& Singer, J. (Eds.) (2001). Handbook of children and the media. Thousand Oaks, CA: Sage Publications.

Steele, V. (1985). Fashion and eroticism: Ideals of feminine beauty from the Victorian Era to the Jazz Age. New York: Oxford University Press.

Strasburger, V. (1995). Adolescents and the media: Medical and psychological impact. Thousand Oaks, CA: Sage Publications.

Strasburger, V. \& Wilson, B. (2002). Children, adolescents, \& the media. Thousand Oaks, CA: Sage Publications.

Striegel-Moore, R. \& Franko, D. (2002). Body image issues among girls and women. In T. Cash \& T. Pruzinsky (Eds.), Body image: A handbook of theory, research, and clinical practice (pp. 183-191). New York: Guilford Press.

Striegel-Moore, R. \& Kearney-Cooke, A. (1994). Exploring parents' attitudes and behaviors about their children's physical appearance. Intemational Joumal of Eating Disorders, 15 (4), 377-385.

Stunkard, A., Sorenson, T., \& Schulsinger, F. (1983). Use of the Danish adoption register for the study of obesity and thinness. In S. Katy, L. Rowland, R. Sidman, \& S. Matthysse (Eds.), The genetics of neurological and psychiatric disorders (pp. 115-120). New York: Raven Press.

Synnott, A. (1992). Tomb, temple, machine, and self: The social construction of the body*. British Joumal of Sociology, 43 (1), 79-110.

Tantleff-Dunn, S. \& Gokee, J. (2002). Interpersonal influences on body image development. In T. Cash \& T. Pruzinsky (Eds.), Body image: A handbook of theory, research, and clinical practice (pp. 108-116). New York: Guilford Press.

Thomas, V. (1989). Body-Image satisfaction among black women. The Joumal of Social Psychology, 129 (1), 107-112.

Thompson, J. \& Altabe, M. (1991). Psychometric qualities of the figure rating scale. International joumal of Eating Disorders, 10 (5), 615-619.

Thompson, J., Heinberg, L., Altabe, M., \& Tantleff-Dunn, S. (1999). Exacting beauty: Theory, assessment, and treatment of body image disturbance. Washington, DC: American Psychological Association. 
Thompson, J. \& van den Berg, P. (2002). Measuring body image attitudes among adolescents and adults. In T. Cash \& T. Pruzinsky (Eds.), Body image: $A$ handbook of theory, research, and clinical practice (pp. 142-154). New York: Guilford Press.

Thompson, J., van den Berg, P., Roehrig, M., Guarda, A., \& Heinberg, L. (2004). The Sociocultural Attitudes Towards Appearance Scale-3 (SATAQ-3):

Development and validation. Intermational Joumal of Eating Disorders, 35 (3), 293-304.

Tiggemann, M. (2002). Media influences on body image development. In T. Cash \& T. Pruzinsky (Eds.), Body image: A handbook of theory, research, and clinical practice (pp. 91-98). New York: Guilford Press.

Tiggemann, M., Gardiner, M., \& Slater, A. (2000). "I would rather be size 10 than have straight A's": A focus group study of adolescent girls' wish to be thinner. Joumal of Adolescence, 23 (6), 645-659.

Tiggemann, M. \& Pickering, A. (1996). Role of television in adolescent women's body dissatisfaction and drive for thinness. Intemational Joumal of Eating Disorders, 20 (2), 199-203.

Tiggemann, M. \& Slater, A. (2004). Thin ideals in music television: A source of social comparison and body dissatisfaction. International Joumal of Eating Disorders, 35, 48-58.

Truby, H. \& Paxton, S. (2002). Development of the children's body image scale. British Joumal of Clinical Psychology, 41 (2), 185-203.

Turner, S., Hamilton, H., Jacobs, M., Angood, L., \& Dwyer, D. (1997). The influence of fashion magazines on the body image satisfaction of college women: An exploratory analysis. Adolescents, 32 (127), 603-614.

TV-Turnoff Network Real Vision (2004, September). Facts and figures about our TV habit. Retrieved September 2004, from http://unw.tvturnoff.org/images/facts\&figs/factsheets/FactsFigs.pdf

TV-Turnoff Network Real Vision (2005, July). Facts and figures about our TV habit. Retrieved July 2005, from http://unw.tvturnoff.org/images/facts\&figs/factsheets/FactsFigs.pdf

Van den Berg, P., Thompson, J. K., Obremski-Brandon, K., \& Coovert, M. (2002). The tripartite influence model of body image and eating disturbance: $A$ covariance structure modeling investigation testing the mediational role of appearance comparison. Joumal of Psychosomatic Research, 53, 1007-1020. 
Van Evra, J. (1998). Television and child development ( $2^{\text {nd }}$ ed.). Mahwah, NJ: Lawrence Erlbaum Associates, Publishers.

Vygotsky, L. (1978). Mind in society: The development of higher psychological processes. Cambridge, MA: Harvard University Press.

Wertheim, E., Paxton, S., Schutz, H., \& Muir, S. (1997). Why do adolescent girls watch their weight? An interview study examining sociocultural pressures to be thin. Joumal of Psychosomatic Research, 42 (4), 345-355.

Wiseman, C., Gray, J., Mosimann, J., \& Ahrens, A. (1992). Cultural expectations of thinness in women: An update. Intemational Joumal of Eating Disorders, 11 (1), 85-89.

Wolf, N. (2002). The beauty myth: How images of beauty are used against women. New York: Perennial. 
Appendix A

Figure Rating Scales 
\#

FIGURE RATING SCALE

Circle the number under the figure that best represents your current body image.

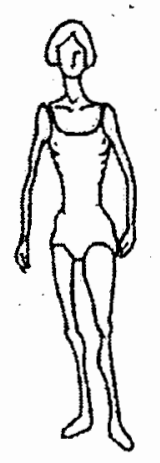

1

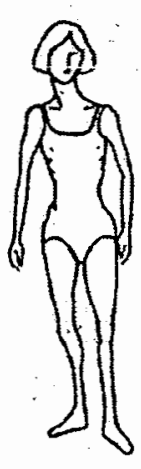

2

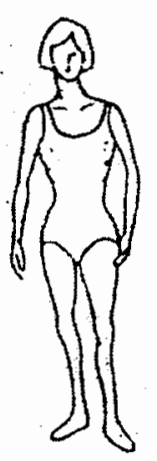

3

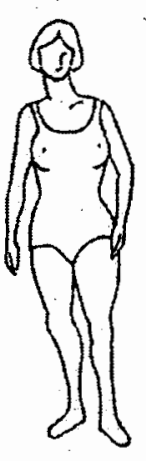

4

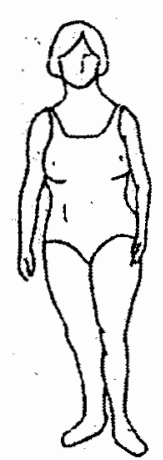

5

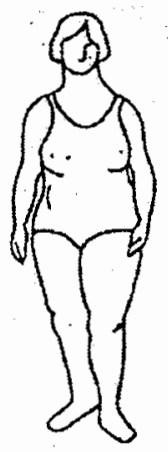

6

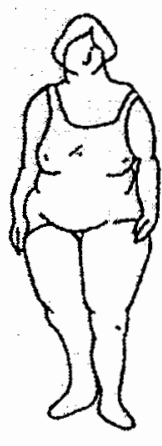

7

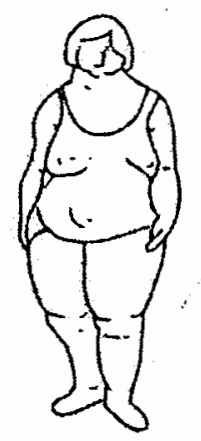

8

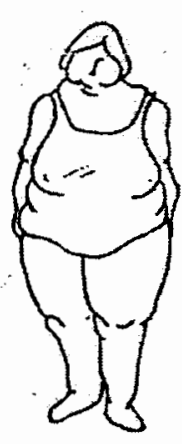

9

(c) Strunkard, AJ, Sorenson, T., \& Schulsinger, F. 
\#

\section{FIGURE RATING SCALE}

Circle the number under the figure that best represents your ideal body image.

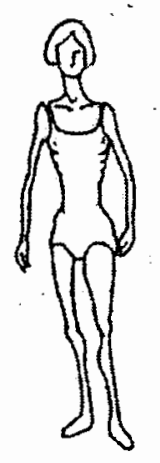

1

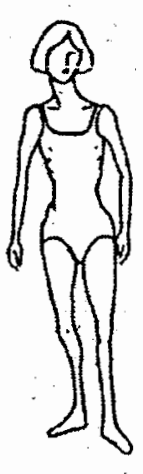

2

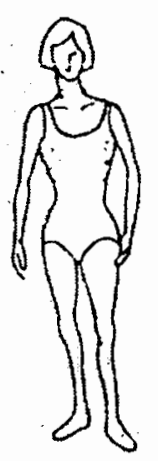

3

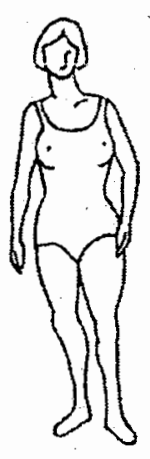

4

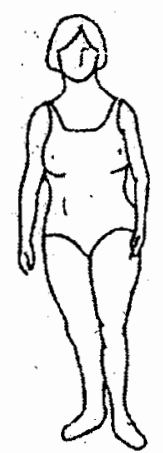

5

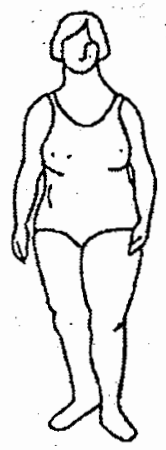

6

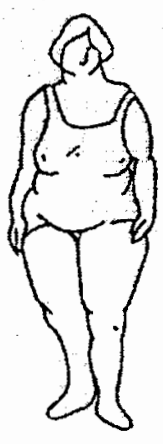

7

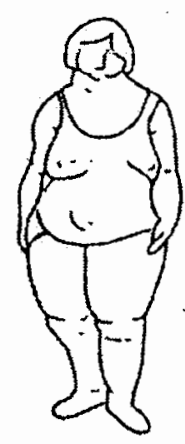

8

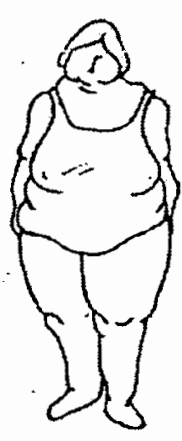

9

CStrunkard, AJ, Sorenson, T., \& Schulsinger, F. 
\#

\section{FIGURE RATING SCALE}

Circle the number under the figure that best represents the media ideal body image.

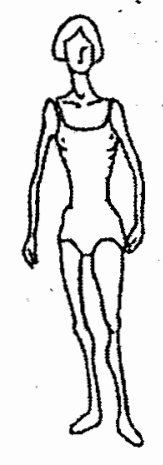

1

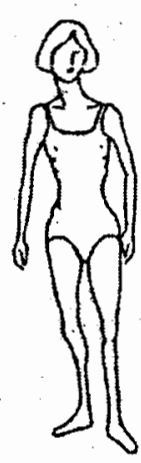

2

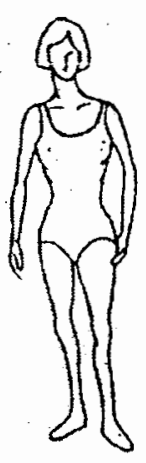

3

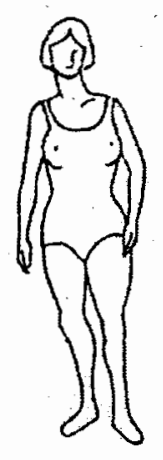

4

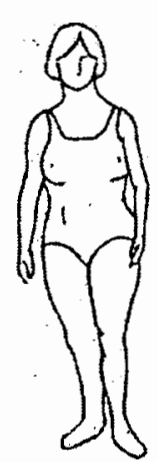

5

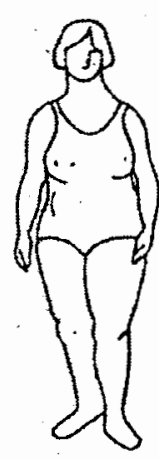

6

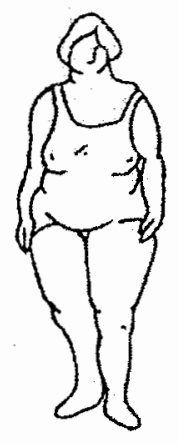

7

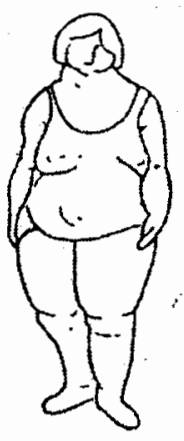

8

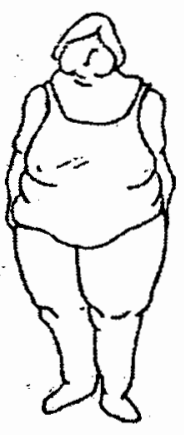

9

(C) Strunkard, AJ, Sorenson, T., \& Schulsinger, F. 
Appendix B

Sociocultural Attitudes Towards Appearance Questionnaire-3

(SATAQ-3) 


\section{\#}

\section{Sociocultural Attitudes Towards Appearance Questionnaire-3}

(SATAQ-3)

Please read each of the following items carefully and indicate the number that best reflects your agreement with the statement.

Definitely Disagree

1

\section{Mostly Disagree}

2
Neither Agree Nor Disagree
3
Mostly Agree $\underset{5}{\text { Definitely Agree }}$

1. TV programs are an important source of information about fashion and "being attractive".

2. I've felt pressure from TV or magazines to lose weight.

3. I do not care if my body looks like the body of people who are on TV.

4. I compare my body to the bodies of people who are on TV.

5. TV commercials are an important source of information about fashion and "being atractive".

6. I do not feel pressure from TV or magazines to look pretty.

7. I would like my body to look like the models who appear in magazines.

8. I compare my appearance to the appearance of TV and movie stars.

9. Music videos on TV are not an important source of information about fashion and "being attractive".

10. I've felt pressure from TV and magazines to be thin.

11. I would like my body to look like the people who are in movies.

12. I do not compare my body to the bodies of people who appear in magazines.

13. Magazine articles are not an important source of information about fashion and "being attractive".

14. I've felt pressure from TV or magazines to have a perfect body. 


\section{Sociocultural Attitudes Towards Appearance Questionnaire-3 (SATAQ-3)}

Definitely Disagree 1

\author{
Mostly Disagree \\ 2
}

Neither Agree Nor Disagree

3
Mostly Agree

Definitely Agree

5

15. I wish I looked like the models in music videos.

16. I compare my appearance to the appearance of people in magazines.

17. Magazine advertisements are an important source of information about fashion and "being attractive".

18. I've felt pressure from TV or magazines to diet.

19. I do not wish to look as athletic as the people in magazines.

20. I compare my body to that of people in "good shape".

21. Pictures in magazines are an important source of information about fashion and "being attractive".

22. I've felt pressure from TV or magazines to exercise.

23. I wish I looked as athletic as sports stars.

24. I compare my body to that of people who are athletic.

25. Movies are an important source of information about fashion and "being attractive".

26. I've felt pressure from TV or magazines to change my appearance.

27. I do not try to look like the people on TV.

28. Movie stars are not an important source of information about fashion and "being attractive".

29. Famous people are an important source of information about fashion and "being attractive."

30. I try to look like sports athletes. 
Appendix C

Body Image/Media Survey 
Body Image/Media Survey

\#:

Directions: Please read each of the following statements/questions carefully and answer each one as it most accurately reflects you and your perceptions.

\section{Self Assessment}

Circle the number that best represents your attitude.

\section{Strongly Disagree Neutral Agree Strongly Disagree Agree}

1. I am satisfied with my body shape.

1

2

34

5

2. I dislike the appearance of my stomach and hips.

1

2

3

4

5

3. I am pleased with how I look in a swimsuit. 1

$\begin{array}{llll}2 & 3 & 4 & 5\end{array}$

4. I am or should be on a diet.

1

2

$3 \quad 4$

5

5. I am satisfied with my weight. 1

2

$3 \quad 4$

5

6. I am displeased with the way clothes fit me.

1

2

34

5

7. Consider the factors that influence your ideas about your body. Rank the following influences on a 1 to 5 scale according to how important each one is in influencing your ideas about your body. Give the most important influence a "1" and the least important influence a " 5 ".

Family/Guardians

Media

Peers

Males

Self 
\#:

\section{Personal History}

Circle the correct response.

8. Have you had any cosmetic surgery (other than your face)? $\mathbf{Y} \quad \mathbf{N}$ If yes, which part of the body and how long ago did the surgery occur?

9. Do you have or have you had an eating disorder? Y N If yes, please list the eating disorder and how long you had or have had it?

\section{Media Assessments}

Circle the number that best reflects your agreement with the statement.

$$
\begin{aligned}
& \text { Strongly Disagree Neutral Agree } \begin{array}{c}
\text { Strongly } \\
\text { Agree }
\end{array} \\
& \text { Disagree }
\end{aligned}
$$

10. The way women appear in music videos influences how I would like to look.

11. The way women appear in magazines influences how I would like to look.

12. The way women appear on television influences how I would like to look. 
\#:

Short answers: Please express your views when answering the following questions. Write legibly!

14. How does the way females appear in media influence how you feel about your body?

15. What is the most powerful message about the female body that media convey to you? Why? 
Appendix D

Demographic Indices 


\section{Young Women's Body Image \\ Study}

Please fill-in the following:

\section{Demographics}

Age: years months

Ethnicity (ies):

Geographic area (only fill-in if you are not from this area):

\section{Personal Information}

Height: feet inches

Weight: pounds 
Appendix E

Letter to Administrators 
Cynthia Cardosi

(434) 296-9048

cc5b@virginia.edu

September 21, 2005

Dear Sir/Madam,

My name is Cynthia Cardosi, and I am a doctoral student at the University of Virginia in Educational Psychology at the Curry School of Education. I am conducting a research study, Young Women's Body Image. I am requesting your permission to recruit 35 young women from your school to participate in this study.

The research study focuses on female adolescents' perceptions of their body image. The participants are young women ranging in age from 18 to 21 years old. This study focuses on late adolescence as the young women are past puberty and their reasoning skills have improved at this stage in their development. In the body image study, I plan to gather the young women's perceptions about their body image and ideal ones. There are three (3) surveys to fill-out for this study: 1) three Figure Rating Scales; 2) the Sociocultural Attitudes Towards Appearance Questionnaire-3 (SATAQ-3); and 3) a Body Image/Media Survey. The timeframe to complete these instruments is approximately 15 minutes.

The packet that accompanies this letter has the materials that will be used in my research study. The packet includes the following forms: (a) study procedures, (b) Informed Consent Agreements for participants, (c) body image definitions, and (d) the participants' survey packet. Please review the materials as well as the approval letter from the University's Institutional Review Board (IRB). I would like to meet with you to discuss your interest in participating in my research study.

The set up that I envision would be an open area where a table and chairs are situated. A sign will be positioned on the table. I would recruit young ladies from that area. The logistics would be worked out with you. My plan is to hopefully collect data during September. Once the young women are recruited, it would take them approximately 15 minutes to fill-in the survey packet. I would need as many days as it would take to collect data from 35 young women.

Any questions that you may have, please feel free to contact me anytime at (434) 296-9048 or cc5b@virginia.edu.

I hope to hear from you and arrange a meeting time at your convenience.

Thank you for your time and consideration.

Respectfully,

Cynthia Cardosi

University of Virginia

Educational Psychology

Doctoral Candidate 
Appendix $\mathrm{F}$

Informed Consent Agreement 


\section{Informed Consent Agreement}

Page 1 of 1

Project Title: The Effect of Media on Female Adolescents' Satisfaction with their Body Image

Please read this consent agreement carefully before you decide to participate in the study.

Purpose of the research study: The purpose of this study is to examine young women's perceptions of their body image. Specifically, the body image study will explore the relationship between your body image and the ideal body images by answering the question of what influences your body image perceptions.

What will you do in the study: You will complete the following three surveys: 3 Figure Rating Scales, Sociocultural Attitudes Towards Appearance Questionnaire-3, and Body Image/Media Survey. You will also provide the following personal data: age, ethnicity(ies), geographic area lived, and height and weight.

Time required: The time required to complete the survey packet is approximately 30 minutes.

Risks: There are no anticipated risks.

Benefits: There are no direct benefits for participating in this study.

Anonymity: Do not put your name on any of the survey materials. Nowhere will your name be revealed or linked with answers given since your name will not be solicited other than signing the Informed Consent Agreement.

Voluntary participation: Your participation in the study is completely voluntary.

Right to withdraw from the study: You have the right to withdraw from this study at any time without penalty.

How to withdraw from the study: If you want to withdraw from the study, you can place the materials in the envelope and tum it in.

Payment: You will receive no payment for participating in this study.

If you have questions about the study, contact:

Cynthia Cardosi

957 Rives Street

Charlottesville, VA 22902

(434) 296-9048

cc5b@virginia.edu

Contact information for Dissertation Chair:

Dr. Harold Strang

Department of Educational Leadership, Foundation, and Policy Studies

Curry School of Education

Ruffner 260

University of Virginia

Charlottesville, VA 22904

(434) 924-0799

If you have questions about your rights in the study, contact:

Luke Kelly, Chairman

Institutional Research Board for the Social and Behavioral Sciences

400 Ray C. Hunt Drive

Suite 360, Room 4

University of Virginia

P.O. Box 800392

Charlottesville, VA 22908-0392

(434) 243-2915

Agreement: I agree to participate in the research study described above.

Signature:

Date: 
Appendix G

Study Procedures 
Young Women's Body Image

Study

\section{PROCEDURES}

Locations:

Post Secondary Schools

PVCC/NCBT/MBC

Participants'

presentations: Individual

Participants' \#s: 40/location

Setting: open area

Materials: Survey packets Site information form

Pencils Procedures

Informed consent forms

Process: Recruit. Interested participants receive an Informed Consent Agreement to sign and an envelope with the survey packet and body image definitions inside.

\section{Inform the young women when recruiting -}

Participants - Your participation is voluntary.

Purpose - This research study focuses on your perception of your body image. This body image study will explore your perceptions about your body image and the ideal ones.

Anonymity - Do not put your name on any of the survey materials. You have complete anonymity as the survey will be returned in the envelope.

\section{Once recruited -}

Consent form - Please sign the Informed Consent Agreement if you agree to participate in this study. Leave the consent form outside of the envelope.

Packets - The envelope contains the survey packet and a list of body image 
definitions. It will take approximately 15 minutes to complete the surveys. There are three (3) instruments to fill-in for this study: 1) three Figure Rating Scales; 2) the Sociocultural Attitude Towards Appearance Questionnaire-3 (SATAQ-3); and 3) the Body image/Media Survey. Select the best answer that reflects how you think and feel. If you are uncomfortable answering a question, then do not answer it. If you have any questions, please ask at any time. Remember you can stop at any time.

When you complete the packet, return it to the envelope and turn it in along with the consent form.

\section{Once the envelope is turned in, inform the participant -}

If you would like to see the results from this study, sign up on this sheet. List your e-mail address or your initial of your first name along with your last name and home address if you have no email address.

Thank you for your time and participation in this study. Any questions? 
Appendix $\mathrm{H}$

Site Information Sheet 


\section{Young Women's Body Image} Study

Location:

Dates:

Administrator:

Sample gathered from:

\# of participants: $1^{\text {st }}$ day -

$2^{\text {nd }}$ day -

$3^{\text {rd }}$ day -

$4^{\text {th }}$ day -

$5^{\text {th }}$ day -

$6^{\text {th }}$ day -

Total numbers of participants:

18

19

20

21

Code numbers to

Times started:

$\begin{array}{ll}\text { am } & \text { pm } \\ \text { am } & \text { pm }\end{array}$

am pm

am pm

Space environment:

Arrangement -

\begin{tabular}{|c|c|c|c|c|c|}
\hline & 1 & 2 & 3 & 4 & 5 \\
\hline Air & & & & & \\
\hline Lighting & & & & & \\
\hline Atmosphere & & & & & \\
\hline
\end{tabular}

Notes: 
Appendix I

Interested in the Results Form 
If you would like results of this study...

Please list only your e-mail address below:

If you do not have an email address then list your initial of your first name along with your last name on the email line and your address on the address line.

Email:

Address: 